\title{
LES \\ FORETTS DU VERSANT SUD DES MONTS DORES \\ Esquisse phytosociologique
}

PAR

\section{Gérard CUSSET}

Assistant en Sorbonte 



\section{AVANT-PROPOS}

Une partie des observations présentées ici ont été faites dans le cadre d'un diplome d'études supérieures soutenu en 1960 à la Faculté des Sciences de Clermont-Ferrand. C"est un agréable devoir pour nous que de remercier tout particulièrement les Professeurs LoISEau et Champaginat qui ont guidé notre travail avec une grande bienveillance et nous ont toujours donné d'excellents conseils,

Ultérieurement de très précieuses discussions avec les Professeurs G. Mangenot et Lemée nous ont apporté des avis particulièrement avertis et nous leur en sommes très reconnaissant. Notre waitre, le Professeur Schneld nous a toujours fait profiter de son expérience et nous a ouvert 'de nouveaux horizons nous permettant une meilleure compréhension de la vie végétale; nous lui exprimons toute notre gratitude.

Nous voulons aussi dire notre reconnaissance à M. d'ALLEIZETTE qui nous a initié à la Systematique et a bien voulu, avec sa compétence habituelle, déterminer nos plantes douteuses ou critiques. Nous devons beatcoup à notre ani B, DE L.ACHAPELLE avec qui nous avons partagé les joies et les difficultés de la recherche sur le terrain. Nous l'en remercions cordialement.

M. I'Ingénieur Principal des Eaux et Forêts Jacamos a bien voulu s'intéresser à nos travaux et at facilité leur publication dans les Annales de l'Fcole Nationale des Eaux et Foréts. Que lui et les membres du Comité de Rédaction de cette publication soient assurés de nos très vif́s remerciements. 


\section{LES FORETS DU VERSANT SUD DES MONTS DORES ESQUISSE PHYTOSOCIOLOGIQUE}

L a diversité géologique et morphologique de l'Auvergne se traduit dans la variété de son revêtement végétal. De nombreux groupements, sotwent morcelés par l'action de l'homme, peuvent y être reconnus. Cependant il semble possible de mettre en évidence quelques grands types de végétation, modulés en plusieurs associations ou sous-associations.

Après l'étude des différents types de hètraies qui constituent la majeure partie des forets naturelles d'altitude, nous examinerons leurs rapports avec les associations non silvatiques voisines et avec les bois de la plaine. Nous envisagerons également le cas iles plantations.

Dans un tel travail il ne nous a pas paru possible d'utiliser uniquement tune phytosociologie statique qui se serait limitée à la description des associations. Nous avons fait un large appel à la pédologie et à la biologie, pour saisir le dynamisme de nos groupements et comprendre leur évolution naturelle. La délimitation précise des différents types de hétraies, généralement imbriqués les uns dans les autres, nous a conduit à l'emploi des mathénatiques statistiques. Ces méthodes variées conduisent à des résultats identiques et ont permis des contróles et des vérifications réciproques. 


\section{GENERALITES}

\section{A - Le cadre géographique et géologique}

\section{1 - Situation.}

Nos relevés ont été effectués sur le versant sud des Dores. Ce massif auvergnat est un important groupe volcanique édifié à la fin du Tertiaire sur les cassures du vieux socle granitique et métamorphique du Massif Central. II domine a l'Est le "graben " de la Limagne, et à l'Ouest le plateau de Latour d'Auvergne incisé des vallées étroites de la Dordogne et de la Burande. Culminant à 1886 mètres au Puy de Sancy, sommet le plus élevé de la France centrale, il comporte bon nombre de pics de plus de 1600 mètres. Nous avons étudié les forèts depuis leur limite supérieure, voisine de 1500 mètres, jusqu'aux environs d'Issoire, dans la Limagne, à environ 400 neetres d'altitude (Carte $n^{\circ} 1$ ). Administrativement, elles font partie du département du Puy-de-Dóme.

\section{2 - Climatologie.}

a) Les vents.

Une étroite bande de hautes pressions, la dorsale de Woiekoff, traverse le massif des Mouts Dores, correspondant à la soudure des masses d'air de Sibérie et des Acores. C"est à cette situation que le climat doit son caractère de grande instabilité. Au nord-ouest de cet axe barométrique, l'afflux aérien provient de l'Océan et la moitié ouest du massif est incluse dans le domaine atlantique. Au sud-est de cet axe, les courants continentaux et les influences méditerranéennes śaffrontent. En réalité, lit zone où se rencontrent influences océaniques et continentales se déplace sans cesse au cours de l'année, et, de façon générale, la fréquence des vents d'ouest est grande. Les yents d'est, plus lents, sont plus sensibles au relief qui les dévie vers la vallée du Rhóne ou le Bassin Parisien.

b) Les précipitations.

Le caractère océanique des Dores est marqué par rapport à la Limagne d'Issoire, tenant en grande partie à la différence d'al- 
titude et d'exposition. Le massif des Dores est en effet le premier obstacle qui se dresse sur le trajet des vents humides atlantiques et les précipitations s'y condensent ahondamment: at Puy de Sancy la moyenne annuelle est très certainement supérieure à 2000 millimètres. Mais à 6 kilomètres seulement, au village du Chambon, elle tombe à $1200 \mathrm{~mm}$ et à Saint-Nectaire, à $15 \mathrm{~km}$, le total annuel n'est plus que de $580 \mathrm{~mm}$. Un tel gradient est exceptionnel. La variabilité est grande: à Besse il a été noté des mois de janvier et février sans la moindre précipitation et d'autres avec un total mensuel supérieur à $300 \mathrm{~mm}$.

L'enneigement est un des traits essentiels du clinat. D'ordinaire, les chutes se produisent entre novembre et avril att-dessus de $700 \mathrm{~m}$ et. au-dessus de $1200 \mathrm{~m}$. I'enneigement est souvent continut de fin novembre à fin avril. A altitude égale, lexposition joue un ròle considérable et au mois d'aout on peut rencontrer des névés dans la vallée de Chaudefour.

\section{c) Brouillards et nébulosité.}

Le véritable brotillard est a une condensation locale de la vapeur d'eau atmosphérique 3. II est fréquent dans la plaine d'Issoire mais devient très rare dès que lon s'élève sur les versants des Monts Dores, ou il apparait comme une nappe de brume stagnant dans les dépressions. Par contre, il est remplacé par des nuages bas tris fréquents. Ils enveloppent les plateaux et les sommets audessus de $1000 \mathrm{~m}$, tandis que tombe une pluie fine trés froide.

l.es observations concernant la nébulosité sont très rares. Sur le versant ouest, à La Bourboule, le ciel est pur ou peu nuageux 170 jours par an. Les jours clairs se répartissent surtout au milieu de I'hiver et au milieu de l'été. Méme quand il a plu l'air ne demeure pas longtemps saturé d'eau: le degré hygrométrique s'abaisse rapidenent et linsolation devient très forte.

\section{d) Les tempíratures.}

Dans lensemble des massifs auvergnats, la température moyenne annuelle est relativement basse. Gachos (1939) cite $10^{\circ}$ a Issoire, $7^{\circ} 6$ à Besse, Flle tombe rapidement à $5^{\circ}$ vers $1600 \mathrm{~m}$. Cependant cette moyenne renseigne mal sur la réalité car le climat est trés contrasté et les écarts de température considérables entre Thiver et l'été. A Besse, la moyenne de janvier est de $-0^{\circ} 5$ et celle de juillet atteint $14^{\circ} 5$. L'amplitude est done de $15^{\circ}$ entre les moyennes mensuelles.

Les variations journalières de la température sont très marquées; en hiver, alors que le soleil chauffe l'atmosphère au-dessus de $20^{\circ}$ l'après-midi, le thermomètre peut descendre à $-20^{\circ}$ la nuit, L'écart atteint alors $40^{\circ}$. 
Le nombre de jours de gelées est important. Durand (1946) l'estime à 150 à $1300 \mathrm{~m}$. Dans la plaine il reste encore important et de l'ordre d'une dizane de jours par an.

\section{c) Conclusions.}

En résumé, le climat des Dores est fait tout de contrastes: " les vents se croisent, s"étagent, se rencontrent ". En quelques heures, le massif passe d'un air polaire à un air méditerranéen. I.es variations rapides du temps commandent tout le devenir de la végétation, très irrégulier d'une année à l'autre.

\section{3 - Géomorphologie.}

Le groupe montrlorien sétend sur $900 \mathrm{~km}^{2}$. II comprend plusieurs centres éruptifs entaillés par des vallées profondes. L'activité de ces volcans s'étant poursuivie pendant une longue période, ils ont émis de nombreuses coulées plus ou moins superposées et souvent enchevètrées. P. GLANGEAUD a divisé en quatre groupes principatux les Monts Dores. Le groupe du Sancy était le plus inportant; disséqué par l'érosion, il est maintenant séparé du groupe ardventif du Barbier par les profondes vallées du Mont Dore et de Chaudefour. Le massif de la Banne d'Ordanche domine La Bourhonle et descend en pente douce jusquà Laqueuille. Le quatricme ensemble, celui de l'Aiguier" ${ }^{* 1}$, affecte la forme d'un vaste éventail rejoignant la limite sud des Monts Dómes.

L.e relief surimposé de ces hauts sommets volcaniques a été érodé tout d'abord par une glaciation de plateau. Après la formation d'une calotte glaciaire continue, qui détermina de nombreux lacs morainiques souvent transformés aujourd'hui en tourbières, linterglaciaire provoqua une érosion fluviatile intense. Deux glaciations de vallées modelèrent ensuite le relief, créant des thalwegs emboités, au caractéristique profil en auge. Ia solifluction joua certainement un róle important après le retrait des glaces, estompant la netteté des reliefs glaciaires. L'érosion des eaux courantes est encore très active. Les couches dures et tendres du sous-sol provoquent un relief en marches d'escalier. L'érosion éolienne est très faible sur les pentes et n'affecte guère que les sommets.

Le réseau hydrographique a la forme étoilée typique des massifs éruptifs. Trois systèmes de cours d'eat rayonnent autour des Monts Dores: la Sioule au nord, la Dordogne à l'ouest et les Couzes au sud-est. Ce massif constitue ainsi la ligne de partage des eaux entre les bassins de la Loire et de la Dordogne.

Il existe de nombreuses sources d'origine hydrothermale qui remontent par les cassures de la "caldeira de Haute-Dotdogne n

* Bien que la carte d'Etat-Major porte Aiguiller, nous préférons Aiguier, conforme à l'étymologie (de \& aigue $>=$ eau). Le sommet est d'ailleurs fort arrondi. 
(Brousse, 1960). Certaines font la réputation de stations thermales, la Bourboule, Saint-Nectaire..., d'autres sont moins connues. Chaudefour, Besse...

\section{4 - Géologie.}

a) Le socle.

I'érlifice volcanique des Monts Dore recouvre un vieux socle cristallophyllien. Par dessus ce socle, un vaste laccolithe de granite s'est injecté en dessous d'une couverture carbonifère. Ces granites hercyniens affleurent à l'entrée de Chandefour. le long d'une faille orientée nord-otrest sud-est. Une récente campagne de sondages électriques a permis de mettre en évidence de nombreuses failles comprises clans tout un système d'effondrement, une caldeira. Nots renvoyons à lat thèse de R. Brousse (Paris, 1960) pour plus de détails.

\section{b) La cowverture voleanique.}

On peut distinguer quatre séries de fornations volcaniques. I.es cinérites inférieures, de hase, présentent une stratification serrée de sables et de tufs. Elles semblent d'âge miocène supérieur d’après une florure de 28 especes. Les formations pliocenes constituent la plus grande partie des Monts Dores. C'est un agglomérat de brèches, de tufs et de cinérites, avec des coulées trachytiques interstratifiées et des dykes nombreux dans la région centrale. II constitue un cone épais de plusieurs centaines de mètres dans sa partie centrale. Ces formations pliocénes sont recouvertes par une véritable carapace de cotulées andésitiques qui descendent en pente douce vers la périphérie du masif. Ce sont ces coulées qui forment la plupart des sommets du massif. Au quaternaire encore, le volcanisme s'est manifesté avec puissance, édifiant un certain nombre de cones et de coulées. Ces dernières se sunt épanchées dans les vallées et sont ainsi à tune altitucle plus faible que les formations antérieures, par inversion du relief.

Notons qu'une interprétation récente (RUDF.. 1962) donne un âge villafranchien (quaternatre) à la plupart de ces formations.

\section{B - Les méthodes d'étude}

\section{1 - La végétation.}

Nous avons employé dans l'étude dé la végétation la méthode codifiée par Josias Braun-Blanguet (Pflanzensociologie, 1951) et connue sous le nom de méthode de Zurich-Montpellier. Nous avons effectué l'étude des individus d'association, sur le terrain, en attachant une grande importance à la notion d'homogénéité floristique de la station. Bien que laire minimale nous ait paru de l'ordre de 
$50 \mathrm{~m}^{2}$, nous avons, dans la mesure du possible, considéré des surfaces de $100 \mathrm{~m}^{2}$ pour l'établissement des relevés. Nous n'avons pas noté isolément les coefficients d'abondance et de dominance et nous avons utilisé les coefficients classiques:

coefficients d'abondance-dominance:

+- nombre d'individus et degré de tecouvrement très faibles

1 = nombre des individus faible ou assez élevé, mais degré de recouvrement faible.

2 - Individus nombreux ou même très nombreux et degré de recouvrement faible

3 = Individus nombreux recouvrant $1 / 2$ de la surface

5 - Espéce numériquement dominante et recouvrant au moins $4 / 5$ de la surface.

coefficients de sociabilite:

1 = Indivirlus isolés

2 - Individus groupés

3 - Individus en troupe

4 - Individus en peuplement

5 - Individus en peuplements denses

La stratification de nos forèts a été traduite par quatre strates:

1 - Strate arborescente, au-dessus de quatre mètres

$\|=$ Strate arhustive

III = Strate herbacée

IV - Strate muscinale (qui peut comprendre des phanérogames de très petite taille ou rampants)

Pour les formes biologiques des plantes, nous avons employé la classification de Raunkiaer (1905):

Phanérophytes - Végétaux arborescents ou arbustifs dont les bourgeons sont à plus de $30 \mathrm{~cm}$ au-dessus du sol.

Chaméphytes - Végétaux dont les bourgeons aériens se trouvent à moins de $30 \mathrm{~cm}$ au-dessus du sol.

Hémicryptophytes - Végétaux dont les bourgeons et les pousses perennantes se trouvent au ras du sol.

Géophytes = Végétaux dont aucune partie ne dépasse le sol pendant la saison défavorable (l'hiver).

Thérophytes $=$ Plantes annuelles passant la mauvaise saison sous forme de graines. 
Une fois établie l'homogénéité des individus d'association, il importe de s'assurer de l'homogénéité statistique des associations et sous-associations quile forment. Du RiETz. Fries et Oswald (1920) ont tracé, les premiers, des graphiques de constance (constance au sens scandinave équivaut à présence). Cependant Augarde (1957). et Gunochet et CASAl (1957) ont montré que a la forme des histogrammes de présence est indépendante de l'homogénéité des tableaux et, a fortiori, des groupements 1". Nons nous sommes donc adressé d'une manière systématique à la méthode de l'analyse différentielle de Czғкалowski. Cette méthode, introduite en France par Gunocuet et fondéc sur les travaux du mathematicien anglais Prarson, est emplové couramment par les phytogéographes d'Furope centrale. Un certain nombre de modifications à la formule initiale ont été utilisées. Les auteurs ne précicant pas toujours celle qu'ils emploient, nous pensons devoir en donner quelques-unes pour permettre des comparaisons.

Soit deux associations $A$ et $B ; a$ le nombre des especes de $A$. $b$ le nombre des especes de $f, c$ le nombre des especes communes à $A$ et $B$.

coefficient de JACCARI (1001):

$$
S=\frac{c}{a+b-c}
$$

coefficient de Kutczysski (1927):

$$
S-\frac{(a+b) \cdot c}{2 \cdot a \cdot b}
$$

coefficient de Sormansen (1948):

$$
S=\frac{2 \cdot c}{a+b}
$$

Nous avons employé le coefficient de Sorensen. Ce coefficient peut ètre calculé pour un nombre quelconque de relevés pris deux à deux, donc caractériser les ressemblances d'un nombre quelconque de relevés. Des hachures conventionnelles traduisent sur un tableau à double entrée les différentes classes de coefficients. Par translation des colonnes et des lignes on range cóte à côte les individus d'association les plus semblables et par là on délimite les associations et sous-associations,

Une fois en possession d'un ensemble homogène de relevés, nous pouvons les comparer entre eux pour dégager l'association plante par plante. Pour conserver le maximum d’objectivité dans la dési- 
gnation des caractéristiques, nous avons employé des tests de signification,

Considérons une plante dans deux séries de relevés, $A$ et $B$, différentielle possible de l'une des séries. Soit $x$ les coefficients d'abondance et $n$ le nombre de relevés. Ia moyenne $\overrightarrow{\mathrm{x}}$ de chaque série est :

$$
\overline{\mathrm{x}}=\frac{\Sigma x}{n}
$$

Ia différence des moyennes est done:

$$
\mathrm{d}=\frac{\sum \mathrm{x}_{\mathrm{a}}}{\mathrm{n}_{\mathrm{a}}}-\frac{\sum \mathrm{x}_{\mathrm{a}}}{\mathrm{n}_{\mathrm{b}}}
$$

La signification statistique de cette différence sobtient en considérant $\sigma_{\mathrm{d}}$ écart-type de la différence

$$
\sigma_{\mathrm{a}}=\sqrt{\sigma_{\operatorname{ma}}^{2}+\sigma_{\mathrm{mb}}^{2}}-\sqrt{\frac{\text { variance de } \mathrm{A}}{\mathrm{n}_{\mathrm{n}}}+\frac{\text { variance de } \mathrm{B}}{\mathrm{n}_{\mathrm{b}}}}
$$

L'estimation de la variance de $\mathrm{A}$ est:

$$
V_{\mathrm{a}}=\frac{\sum\left|\mathrm{x}_{\mathrm{n}}-\overline{\mathrm{x}}_{\mathrm{a}}\right|^{2}}{\mathrm{n}_{\mathrm{a}}-1}
$$

Par ailleurs nous pouvons écrire:

$$
\mathbf{\Sigma}\left|\mathrm{x}_{\mathrm{a}}-\overline{\mathrm{x}}_{\mathrm{a}}\right|^{2}-\mathbf{\Sigma}\left(\mathrm{x}_{\mathrm{a}}{ }^{2}\right)-\frac{\left[\mathbf{\Sigma}\left(\mathrm{x}_{\mathrm{a}}\right)^{2}\right]}{\mathrm{n}_{\mathrm{a}}}
$$

L'estimation de l'écart-type de la différence est alors:

$$
\mathrm{S}_{\mathrm{a}}=\sqrt{\frac{\mathrm{n}_{\mathrm{a}} \sum\left(\mathrm{x}_{\mathrm{a}}{ }^{2}\right)-\left(\sum \mathrm{x}_{\mathrm{b}}\right)^{2}}{\mathrm{n}_{\mathrm{a}}{ }^{2}\left(\mathrm{n}_{\mathrm{a}}-1\right)}+\frac{\mathrm{n}_{\mathrm{b}} \sum\left(\mathrm{x}_{\mathrm{b}}{ }^{2}\right)-\left(\sum \mathrm{x}_{\mathrm{b}}\right)^{2}}{\mathrm{n}_{\mathrm{b}}{ }^{2}\left(\mathrm{n}_{\mathrm{b}}-1\right)}}
$$

Les variances $\sigma_{\mathrm{ma}}{ }^{2}$ et $\sigma_{\mathrm{mah}}{ }^{2}$ étant a priori inconnues, deux cas peuvent se présenter:

a) cette formule est applicable quand $n_{\mathrm{i}}$ et $n_{\mathrm{b}}$ sont grands (au moins une quarantaine de relevés) : on peut alors assimiler l'estimation de l'écart-type à la véritable valeur de cet écart-type. Il suffit de comparer $\mathrm{R}-\frac{\mathrm{d}}{\mathrm{S}_{\mathrm{a}}}$ à la valeur correspondant à la probabilité 
désirée de la loi de distribution normale. Nous choisirons une prolaabilité de $95 \%$; il suffira done de comparer la valeur de $R$ à 2 valeur du $\mathrm{t}$ de STUDent-Fisher pour un seuil de probabilité de 0,05.

b) $n_{a}$ et $n_{b}$ sont inférieurs à quarante. Ce sera ici le cas général. On peut supposer sans difficulté que les deux échantillons, c'està-dire les deux séries de relevés, sont issus de populations normales, et il faut aussi supposer que les variances de $A$ et de $B$ sont égales. Comme il s'agit d'échantillons très semblables, cela est très probable dans les cas retenus ici. () estime la valeur de la variance à :

$$
V_{a}-V_{b}-S^{2}-\frac{\sum\left(x_{a}-\vec{x}_{a}\right)^{2}+2\left(x_{b}-\bar{x}_{b}\right)^{2}}{n_{n}+n_{b}-2}
$$

On a :

$$
S_{d^{2}}=\frac{S^{2}}{n_{n}}+\frac{S^{2}}{n_{b}}=S^{2} \frac{n_{u}+n_{2}}{n_{n_{n}} n_{b}}
$$

Le rapport Ke est alors égal à :

$$
R=\frac{d}{S_{d}}=d \sqrt{\frac{n_{a}+n_{b}-2}{\Sigma\left(x_{a}-\bar{x}_{a}\right)^{2}+\Sigma\left(x_{b}-\vec{x}_{b}\right)^{2}}} \cdot \frac{n_{a} \cdot n_{b}}{n_{a}+n_{b}}
$$

Et il est possible de comparer la valeur de $k$ a la valeur de $t$ donnée par la table de Student-Fisher à $\mathrm{N}-n_{n}+n_{\mathrm{b}}-2$ degrés de liberté, et au seuil de probabilité que nous avons choisi égal ì 0,05 . Si $R \geqslant t$, la plante est différentielle avec une probabilité de $95 \%$ de la série où elle est la plus abondante. Notons immédiatement que les résultats écologiques et biologiques confirment pleinement les mises en évidence ainsi réalisées. Cette méthode que nous nous proposons d'appeler "méthode de la différenticlle statistique ", autorise évidenment des séparations qu’il serait fort délicat d'opérer sans sappuyer sur me lase mathématique.

Une fois les caractéristiques et les différentielles reconnues, on les utilise pour la dénomination des associations selon la méthode de l'Ecole de \%urich-Montpellier. Notons dès maintenant que les dénominations attribuées à certaines entités d'ordre supérieur (alliance, ordre) pourraient surprendre. C'est ainsi que nots allons être appelé à classer dans le Quercion robori-sessiliflorae des forèts ne renfermant pas trace de chènes. II s'agit là d'une stricte question de nomenclature. Remarquons par ailleurs que pour le phytosociologue, à l'encontre parfois du forestier, les arbres ne constituent qu'un élément d'une forèt, le plus spectaculaire sans doute, mais pas toujours le plus important au point de vue association. Un bois de hêtres dont le sous-bois serait formé de plantes 
appartenant au cortège habituel du chène sessile sera considéré comme une chênaie par le phytusociologue, et réciproquement. " II ne faut pas confondre forèt de hêtres et association du hêtre " (Braun-Blanguet).

Il est parfois difficile de ranger certaines associations des hẻtraies dans les cadres phytosociologiques d'ordre supérieur. Nous proposons une représentation graphique qui nous l'a permis assez simplement; toute forèt des Querceto-Fagetea peut en effet ètre représentée par un point dans une figuration géonétrique et l'emplacement de ce point est caractéristique de sa position dans la classification.

Soit une foret des Querceto-Fagetea; c'est l'ensemble de quatre groupes de plantes:

(a): Plantes du Fagion et des Fagetalia silvaticae

( $\beta$ ): Plantes du Quercion et des Quercetalia robori-sessiliflorae

$(\gamma)$ : Plantes des Querceto-Fagetea

(d) : Especes compagnes

On peut donc caractériser la position systénatique de cette association par quatre chiffres:

$$
\mathrm{x}=\frac{\alpha}{\gamma} \quad y-\frac{\gamma}{\delta} \quad z-\frac{\delta}{\alpha} \quad \mathrm{t}=\frac{\alpha}{\beta}
$$

$\alpha, \beta, \gamma$, d étant respectivement la somme des moyennes $\overrightarrow{\mathrm{x}}$ des plantes de chaque ensemble $(\alpha),(\beta),(\gamma),(\delta)$.

Par ailleurs $\alpha+\beta+\gamma+\delta=\mathrm{N}, \mathrm{N}$ étant un nombre fixe. N'ayant que trois degrés de liberté pour nos variables, nous pouvons donc envisager une figuration spatiale à trois dimensions. Consiclérons un tétraèdre régulier $\mathrm{ABCD}$ (fig. 1). En coordonnées triangulaires dans le triangle équilatéral $\mathrm{ADC}$ on peut représenter $\mathrm{x}, \mathrm{y}, \mathrm{z}$, par des points L, M, N, tels que

$$
x=\frac{C L}{D M}=\frac{C L}{A M^{\prime}} y-\frac{D M}{A N}-\frac{D M}{C N^{\prime}} z-\frac{A N}{C L}=\frac{A N}{D L^{\prime}}
$$

La solution existe et elle est unique. Les paralleles à CD, DA et $\mathrm{AC}$ menées respectivement par $\mathrm{L}, \mathrm{M}$, et $\mathrm{N}$ sont concourantes en un point $\mathrm{O}$. Ce point $\mathrm{O}$ est le barycentre de A,D,C affectés de $\alpha, \delta$ et $\gamma$. On finit de caractériser lassociation avec $\Omega$ tel que $\mathrm{B} \Omega$

$t-\frac{\Omega}{\Omega O}$. Ce point $\Omega$ est la représentation de l'association. 
Si $\Omega$ est à l'intérieur du trone de pyramide ADCHKJ*, l'association fait partie des Fagetalia. Si Q est à l'intérieur de la pramide BHKJ, l'association fait partie des Quercetalia. In outre, la position de $O$ montre l'importance relative des especes compagnes, c'est-à-dire dans une certaine mesure, l'état d'intégrité de l'association.

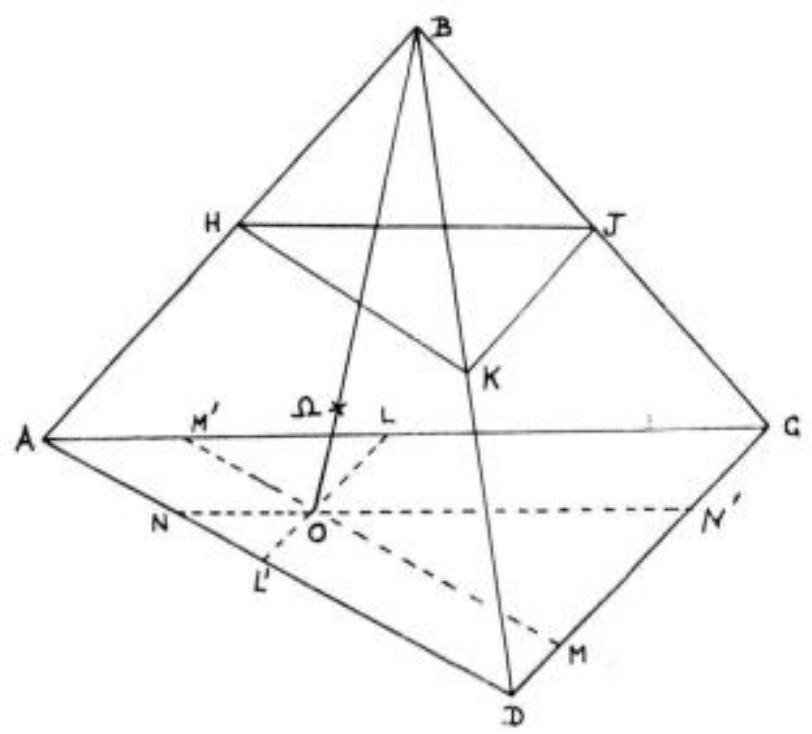

FIG. 1.

Il est très facile de représenter la pyramide ABCD en projetant sur les plans des triangles $\mathrm{ADC}$ et $\mathrm{ABD}$.

Pratiquement on considere ces triangles; on ramène $\alpha+\gamma+\delta$ d̀ 100 ; et on calcule $\alpha, \gamma$ et $\delta$ en pourcentage. Soit a, c et d les valeurs obtenues. On porte $\mathrm{AN}-\mathrm{d}, \mathrm{CL}-\mathrm{a}$, et $\mathrm{DM}=\mathrm{c}$ sur les cótés AD, CA et DC divisés en 100 parties égales. On construit () graphiquement. Pour avoir \& on rabat $O$ et on trace $Q$ tel que $t=\frac{\mathrm{B} \Omega}{\Omega O}$.

Cette méthode nous a également servi à délimiter certains groupements de prairies et nous pensons que son emploi pourrait ètre généralisé, malgré l'inconvénient des calculs.

- $\mathrm{H}$, J et $\mathrm{K}$ sont respectivement les milieux de $\mathrm{AB}, \mathrm{BC}$ et $\mathrm{BD}$, cest-adire cu'ils délimitent par le plan HJK la portion de la pyramide où l'influence de B (Quercion) est supérieure à celle de A (Fagion) C (Querceto-Fagetea) et D (Espèces compagnes). 


\section{2 - Le milieu.}

Nous examinerons successivement les méthodes d'ètudes des milieux climatique, pédologique et biotique.

\section{a) Les facteurs climatiques.}

l.e climat régional étant supposé connu, il s'agit de déterminer le méso-climat et les micro-climats propres à chaque association et partic d'association.

Ne disposant pas d'appareils enregistreurs, nous avons fait des mesures à l'aide d'un thermomètre à mercure gradué en cinquièmes de degrés Celsius. Nous avons protégé le réservoir par une rondelle de carton blanc réfléchissant le rayonnement solaire direct.

La luminosité a été mesurée, par comparaison elle aussi, à l'aide de deux appareils, une cellule photoélectrique et un posophotomètre à comparaison de brillance. Les résultats obtenus sont homologues.

I.'humidité relative de l'atmosphère a été relevée à l'hygromètre à cheveu, soigneusement réglé chaque fois. Nous en avons déduit, de façon approchée, le déficit de saturation. II donne en effet le rapport $\mathrm{h}=\frac{\mathrm{f}}{\mathrm{F}}, \mathrm{f}$ étant l'humidité réelle et $\mathrm{F}$ la pression saturante de vapeur d'eau dans les mèmes conditions. On en déduit $\mathrm{e}=\mathrm{F}(\mathrm{I}-\mathrm{h})$. F est fourni par les tables de Regnault (voir par exemple Angot - Introduction à la Météorologie).

L'évaporation a été mesurée avec des évaporimètres de P'iche, simultanément en des endroits différents.

h) Les facteurs pédologiques.

Deux temps principaux s'imposent: sur le terrain, étude des profils des sols et de certaines propriétés; au laboratoire, analyses et détermination des autres caractéristiques. Nous avons employé la terminologie proposée par Aurert et Duchaufour (Congrès de Paris, 1956). Voici la nomenclature utilisée:

$\begin{array}{cl}\begin{array}{c}\text { horizons } \\ \text { sans entrainement } \\ \text { (sols peu évolués) } \\ \begin{array}{c}\text { ou horizons éluviaux } \\ \text { (sols évolués) }\end{array}\end{array} & \mathrm{A}_{0} \text { matières organiques pures } \\ \begin{array}{c}\text { horizons illuviaux } \\ \text { d'accumulation }\end{array} & \mathrm{A}_{2} \text { minéral } \\ & \mathrm{B}_{1} \text { riche en argiles et en fer } \\ & \mathrm{B}_{2} \\ \text { présence } & \mathrm{G} \text { gley } \\ \text { d'une nappe d'eau } & \mathrm{C}\end{array}$


Nous n'avons pu effectuer en laboratoire que quelques mesures. en particulier granulométriques. Voici brièventent notre mode opératoire:

La fraction supérieure à $2 \mathrm{~mm}$ est d'abord séparée par tamisage et pesée.

La fraction inférieure est conservée pour la suite des opérations. Les matières organiques sont dosées par calcination au rouge et addition d'eat oxygénée concentrée. sur $10 \mathrm{~g}$ de sol séchés à l'air.

La granulométrie est effectuée sur un autre échantillon de $10 \mathrm{~g}$. après élimination des matières organiques. La dispersion des colloïdes est assurée par adjonction de citrate de soude sodique. Une sédimentation de huit heures pour $10 \mathrm{~cm}$ de chute fournit cleux fractions séparées par siphonage:

- suspension de colloïdes minéraux et organiques

- dépôt des sables et des silts (- limons)

L.es sables sont ensuite séparés des silts par une sédimentation de $14^{\prime} 30^{\prime \prime}$ pour une hauteur de $30 \mathrm{~cm}$ de chute. Après siphonage. ces deux éléments sont séchés à l'air et pesés.

La suspension est alors reprise: l'addition d'une solution de chlorure de potassium et le chauffage ménagé provoquent la floculation des colloïdes minéraux (= argiles) qui sont recueillis par filtration sous un léger vide. L'addition d'acide chlorhydrique concentré au filtrat fait alors floculer les colloides humiques qui à leur tour sont filtrés, séchés et pesés.

Les divers résultats sont exprimés en pourccutage de terre fine séchée à l'air.

I.es procédés, parfois rudimentaires, employés n'ont pas permis une grande précision et lerreur peut atteindre 6 à 7 pour cent. Pour que le lecteur puisse apprécier le degré d'erreur. nous n'avons en aucun cas "arrondi " les calculs pour ohtenir un total de 100 .

\section{c) Les facteurs hiotiques.}

Nous n'avons effectué aucune culture de la microflore du sol, mais nous avons eu la chance de bénéficier, sur notre terrain, des ohservations et des conseils de Th. Dominik et de B. Boultarn, spécialistes de la thizosphère. qui nous ont fourni de très précieux éléments.

Nous avons fait appel à quelques traits biologiques d'observation, notamment pour expliquer les divers moles de concurrence vitale et les relais d'espèces lors de la dynamique de nos associations. T.es modes de dispersion des espèces et leur facilité de survie à d'anciens épisodes climatiques défavorables sont également importants À considérer. 


\section{LES FORETS DU VERSANT SUD DES MONTS DORES}

On appelle " hètraie ", au sens usuel du mot, un bois constitué essentiellement de hêtres. Au sens phytosnciologique, dacception quelque peu différente, c'est une association végétale renfermant un certain nombre de caractéristiques dont le hètre constitue la plus spectaculaire. Dans le cadre de la région étudiée ici, cette distinction ne joue guère et nous appliquerons indifféremment le terme de hetraic à des ensembles répondant à l'une ou à l'autre de ces définitions.

Dans le massif des Dores, la hêtraie semble ètre l'un des climax, et dans ce pays rude, souvent d'accès pet facile, certaines parties de la vaste forèt primitive ont pet subi l'action de l'homme. Elles nous présentent done l'aspect naturel de la végétation indigène, dans si variété de détails originelle. Cette diversité se traduit par les compositions différentes du tapis herbacé, reflets de l'état du sol et de l'état biologique de la forêt. Nous allons examiner successivement:

A - les hêtraies de l'ordre des Fagetalia (à sol peu acide),

B les hètraies de londre des Quercetalia (à sol acide).

C le milieu de la hètraie (facteurs climatiques, édaphiques et biotiques),

1) les rapports de la hêtraic avec les associations non silvatiques voisines.

$\mathrm{F}$ - les rapports de la hétraie avec les forèts d'altitude moindre.

F - les plantations et leur avenir possible.

\section{A - Les hêtraies de l'ordre des Fagetalia}

\section{1 - Le Fageto-Scilletum lilio-hyacinthii Lemée 1956.}

I,UOUET, dans sa thèse (1926). est le premier auteur à avoir envisage l'etude des forets d'Auvergne sous un angle phytosociologique. Il ne faudrait cependant pas oublier les très exactes descriptions de LEcoQ dès 1835. En 1932, le fondateur de la méthode Zuricho-Montpelliéraine, Braun-Blanouet, désignait le climax des hêtraies d'Auvergne sous le nom de Fagetum gallicum. I1 nous semble cependant que les trois relevés de notre région donnés par cet auteur ne peuvent être rangés dans le Fagetum gallicum tel qu'il devait lui-mème le définir en 1952 ; peut-être sont-ils plus proches du Fageto-Scilletum (Br.-BL.) des environs de Luchon. En 
1956, LEM ÉE devait proposer le terme, conforme à la nomenclature, de Fageto-Scilletum lilio-hyacinthii pour " la hétraie à sol brun à humus du type mull a du Massif Central. Ce terne convient parfaitement à certaines hêtraies des Monts Dores et nous ladoptons, dans un sens peut-ètre un peut plus restreint, car nous en écartons les hètraies à luzules.

Cette association se présente sous la forme d'une belle futaie où le hêtre, presque exclusif dans la strate arborescente, est particulièrement bien venu. I a forèt n'a qu'une strate arbustive très réduite, avec, de ci de là, quelques jeunes hêtres, quelques alisiers. ronces et Lonicera (nigra et alpigcna). Nos relevés ont pu aisément porter sur des individus d'association oi aucune coupe n'avait été effectuée depuis fort longtemps, car l'exploitation de ces bois est souvent très irrégulière et très espacée dans le temps. Certains relevés concernent des futaies dont les arbres avaient gelé à cause de l'altitude. Leur tronc avait séché et ils avaient repoussé à partir de rejets. Nous n'avons rencontré ce phénomène que dans cette association - où les conditions sont optimales pour le hêtre.

L'altitude où l'association atteint son optimum est d'environ $1300 \mathrm{~m}$ et les individus d'associations bien caractéristiques que nous avons rencontrés s'échelonnent de 1100 à $1450 \mathrm{~m}$. Ia pente du sol est souvent forte. $40^{\circ}$ en moyenne et nous avons noté une pente extrême de $60^{\circ}$ dans la vallée du Chaudefour. Cette difficulté d'exploitation n'est nas sans expliquer le peu d'action de l'homme que présentent ces forêts. I.e substratum est très varié, comprenant des granites, des trachytes, des andésites et des basaltes. Il ne parait pas avoir d'action marquée sur la composition floristique ou la croissance rle la forêt. De même l'exposition ne semble pas préférentielle, bien que les vallées glaciaires et fluviales dont lepentes les portent soient généralement orientées d'ouest en est.

Nous avons assuré l'homogénéité de notre tableau de relevés far la méthode de Czenanowski. I.e coefficient de Sorfansen n'est jamais descendu en dessous de 35 , ce que nous considérons comme suffisant*.

* Le tableau complet et le tahleau des coefficients de Czekanowski sont portés dans le mémoire déposé au Laboratrire de Botanique de la Faculté des Sciences de Clermont-Ferrand. II en est de même pour les autres associations. De la mème façon nous n'avons pu indiquer ici les coefficients d'ahondance-dominance. Pour permettre une connaissance suffisante des Associations, nous avons noté les associations en coefficients de présence et en indice de classe.

Coefficient de présence (1 ${ }^{\text {or }}$ chiffre noté):

$I=$ Espèce très rare.

II = Espèce représentée dans moins de $2 / 5$ des relevés.

III = Espèce représentée đans $2 / 5$ à $3 / 5$ des relevés.

IV $=$ Espèce représentée dans $3 / 5$ à $4 / 5$ des relevés.

$\mathrm{V}=$ Espèce représentée dans $4 / 5$ at moins des relevés.

Indice de classe (2* chiffre noté):

L'indice de classe est le rapport de la somme des coefficients d'abon-

dance-dominance au nombre de présence. 
L'association ainsi constituée, nous avons élevé neuf espèces au rang de caractéristiques d'association. L.eur présence habituelle, aussi bien dans l'association typique que dans les sous-associations nous y a autorisé. Leur aire restreinte et leurs conditions écologiques définies assez strictement nots permettront de les considérer comme différentielles de notre association, par rapport aux autres associations du Fagion lorsque nous comparerons cette hêtraie aux autres hêtraie françaises et européennes.

\section{Caractéristiques d'Association.}

Fagus silvatica I. V; 3,0 - Scilla lilio-hyaciulhus I.. V; 1,5 - Fuphorbia hyberna L. IV: 0.6 - Fasuss silatica L. (germinations) IV : 0,3 - Stcllaria nemorum L. ssp. glochidisperma Murb. III; 0,6Pulmonaria affinis Jordan ssp, eu-affinis P. F. III;0,3 - Polygonatum verticillatum AII, III ; 0,3 - Fagus silvatica I. (arbustif) II : 0,4 - Epilobium montanum L.II 0.3 - Circaca intermedia Fhrh. I; 0,1 - Conopodium denudatum Koch $\mathrm{I}: 0,1$.

\section{Fagion et Fagetalia silvaticac.}

Asperula odorata L. IV; ì,6 - Actaca spicata I. IV :0,4 - Paris quadrifolia L. IV: 0,3 - Galeobdolon huteum Huds. IV : 0,2 - Milium effusum L. IV : 0.2 - Prenanthes purpurea L. III : 0.7 - Myosotis silaratica Hoffm. III : 0.4 - Phyteuma spicatum I., ssp. ochroleucum Schulze III : 0,1 - Dentaria pinnata I.mk. II ; 0,8 - Festuca silvatica Vill. II : 0.3 - Euphorbia dulcis I. II : 0.1 - Euphorbia anygdaloides L. II $: 0,1$ - Cicerbita muralis Wallr. I : 0,1 - Lilium martagon L. I: 0.1 - Neotfia nidus-avis L. I: 0.1 - Poa nemoralis L. I; 0.1 -Carex silzatica Huds. I; 0,1 - Impatiens noli-tangere $\mathrm{T}$. $\mathrm{I}: 0.1$ - I.ysimachia nemorum I. $\mathrm{T}: 0,1$ - Epilohimm durieui Gay I : 0.1 - Scrophularia nodosa I. I: 0,1 - Carex remota I. I : 0,1,

\section{Quercion et Quercetalia robori-sessiliflorac.}

Solidago virga-aurea L. II : 0.2 - Convallaria maialis I. II: 0.2 Hicracium groupe murormm I; 0,3-Deschampsia flexuosa Griseb. $\mathrm{I}$ : 0.1 - Sorbus aria Crantz $\mathrm{I}: 0.1$ - Maianthemum bifolium $\mathrm{D}: \mathrm{C}$. I: 0,1 .

\section{Querceto-Fagetca.}

Viola silvestris Koch IV: 0.5 - Ancmone nemorosa I.. IV: 0.3 Melandryum silvestre Roehl IV: 0,2 - Melica uniflora Retz III : 0,1 - Moehringia trinervia Clairv. II ; 0,2 - Abies pectinata Lmk. I; 0,1 Ranunculus breyninus Crantz $\mathrm{T}: 0,1$ - Bromus asper L. I: 0,1 Arum maculatum L. I: 0,1 - Epipactis latifolia Vill, I: 0,1 - Campanula trachelium L. I; 0,1 - Senecio spathulaefolius D.C. I; 0,1. 


\section{Espèces compagnes.}

Oralis acetosella I. III; 0.4 - Dryopteris fitix-mas Schott. III ; 0,4 - Athyrum filix-focmina Roth. III 0,4 - Poa chairii Vill. II ; 0.4 - Sencio cacaliaster L.mk. II : 0,2 - Luzula maxima D.C. I 0.3 Pheyopteris dryopteris Fée I; 0.1 - Geranium robertiamum L. 1; 0,1 - Sorbus aucuparia I.. I ; 0,1 .....

\section{Muscinées.}

Hylocomium triquetrum $\mathrm{Br}$. Eur. 1; 0,1 - Hylocomium splendens Br. Eur, I; 0.1 - Polytrichum formosum Herw, I; 0.1 ...

(Association définic sur 28 relevés).

Scilla lilio-hyacinthus I. endémique pyrénéo-centrale est la meilleure de nos caractéristiques; nous ne l'avons rencontrée qu'une fois en dehors des hètraies sur le flanc sud du Puy de Pallaret. Euphorbia hyberna L., espéce strictement atlantique, est ici à sa limite orientale et fournit une bonne caractéristique des forèts du Massif Central et des Pyrénées. Au contraire, la sous-espece glochidisperma Murb. de Stellaria nemorum I. est plutôt nordique (voir I.awalrée). Non encore signalée dans notre région, elle semble avoir été méconnue car elle est bien caractérisée, et la confrontation avec des specimens d'herbier nous l'a confirmé. Pulmonaria affinis Jordan, sous-espèce cu-affinis P.F. est plutòt une plante du sud de la France, et on ne la rencontre en Auvergne que dans les bois des Fagetalia, station assez étonnante pour une plante méridionale; il est vrai que la systématique très embrouillée đles Pulmonaires ne correspond peut-être pas à la réalité écologique. Polygonatum verticillatum Allioni se rencontre à l'étage du hêtre sur sol brun des forèts et apparait comme une caractéristique régionale. Efilobium montanum I.. sensu stricto est fréquente dans notre dition alors qu'Epilobium durieur Gay ne se trouve qu'à leur limite supérieure. Une autre Oenotheracée, Circaea intermedia se montre fréquenment dans les stations oì la nappe phréatique est proche de la surface, de même que Conopodium donudatum Koch, atlantique à sa limite est.

Cette association est commune dans les Monts Dores où elle conserve une bonne homogénéité floristiqute. L'ensemble caractéristique est pratiquement toujours présent. Les individus d'association peuvent recouvrir des surfaces continues de l'ordre d'un hectare ou ètre morcelés en fragments de quelques mètres carrés. Dans les Monts Dômes elle est beaucoup plus dégradée et n'est restée à l'état de futaie que dans le parc d'Allagnat et sur les. Puys de Cóme et de Manson. La très belle carte de la végétation des Monts Dómes dreszée par LemḱE en montre la répartition exacte. Daphne mezerewm I. y semble plus fréquent que dans les Monts Dores. La hêtraie des Gardes dans les Monts de la Madeleine marque la limite 
nord-est de nos deux caractéristiques les plus importantes, Scilla lilio-hyacinthus et Euphorbia hyberna. I.e cortege floristique du hêtre $y$ est appauvri, et la hêtraie passe insensiblement au Querceto-Fagetum LeméE 1937. Le Forez ne possède pas les caractéristiques atlantiques de notre association, et les hêtraies de cette région paraissent proches de celles décrites dans les Cévennes par Braun-Blanguet comme Fagetum gallicum conopodietostm (1953). Par contre, les hêtraies du Cantal sont identiques à celles des Dores. avec lesquelles elles sont en continuité plus ou moins nette. II en est de même pour celles de la partie ouest de l'Auvergne et de 1"Artense. Noton cependant que le houx y semble plus abondant.

11 est permis de penser qu'on rencontre le Fageto-Scilletum liliohyacinthii dans les Pyrénées, bien quue nous ne possédions pas de relevés de cette région. Il y aurait cependant lieu de distinguer deux ensembles à nombreuses différentielles, les Dores ne possérlant évidemment pas les nombreuses endémiques des Pyrénées.

\section{2 - Le Fageto-Scilletum athyrietosum.}

Nous avons proposé la dénomination de Fageto-Scilletum athyrietosum, en 1960, pour désigner un groupement assez fréquent dans les hêtraies des Dores. Il se présente généralement sous forme fragmentaire le long des ruisselets ou dans les éboulis au pied des falaises trachytiques. Nous avons cependant rencontré des surfaces notables recouvertes par cette sous-association notanment dans les bois de Chaudefour et de Sarrevielle.

Le plus souvent il s'agit d'un taillis-sous-futaie, Iat strate arborescente n'a pas un recouvrement supérieur à $55 \%$. et les hêtres qui la composent sout de faible taille. souvent tortueux et mal venus. Parfois cependant its peuvent pousser de meilleure façon. à Chaudefour surtout. La strate arbustive est formée de hêtres jeunes et de rejets, avec quelques sorbiers. Les fougères et de grandes composées encombrent le sous-bois. I a strate herhacée est très vigntreuse. les mousses rares.

Cette sous-association atteint son optimum de développement sensiblement à la mème altitude que l'association, 1300 à $1350 \mathrm{~m}$, mais elle descend nettement moins bas et monte relativement très haut près de la limite supérieure de la forèt. Ia pente du sol est particulierement forte. ce groupement occupant les rebords glaciaires raides des vallées en auge. Le substratum géologique est volcanique. et comprend des laves variées, acides ou non.

Un trait important de cette sous-association tient à son exposition. Pour 6 expositions est, 9 nord-est et 3 nord dans les indivirlus d'association typiques considérés, aucune exposition ouest, sud-ouest ni sud. Les conditions écologiques nous en donneront la raison. L'homogénéité statistique du tableau de sous-association a été fournie 
nar la méthode de Sorensen. I.es coefficients ont varié de 32 à 62 . Aucune structure n'a été mise en évidence dans le tableaut de CzEkANowski.

Différenticlles de sou.s-a.ssociation.

Athyrium filix-foemina Roth. V: 1,4 - Dryopteris filix-mas Schott. $\mathrm{V} ; 1,3$ - Mclandryum silvestre Roehl. V: 0,7 - L.ysimachia nemormm L. V: 0,4 - Carex leporina $\mathrm{I}$. et var, argyroglochin Koch) III;0,1 Aspidium aculcatum Sw, ssp. angulare Kit, II: 0.2 - Polistichum spinulosum D.C. ssp. cu-spinulosum Mill. II: 0.3 .

Caractéristiques d'association.

Faqus silvatica I. V: 2.4 - Fagus silvatica I. (arbustif) V:0,5 Scilla hilio-hyacinthus I.. IV:0,6 - Fagus silatica I. (germinations) III $; 0.7$ - Epilobium montanum I. III : 0.2 - Pulmonaria affinis Jord. ssp. cu-affinis P.F. II : 0.3 - Circaca intermedia Ehrh. II 0.2 Euphorbia hyberna L. II: 0,2 - Stcllaria nemorum I. ssp. glochidisperma Murb. II; 0,1 - Polygonatum verticillatum AII. N:0,1 Pulmonaria affinis Jord. ssp. alpestre Lam. I; 0.1.

Transgressizes de l'Adenostylion.

Mulgedium alpimum Less. NN; 0.1 - Adenostyles albifrons Rchb. II : 0,2 - Doronicum austriacum Jacq. 1:0.1 - Mulgedium phrmicri D.C. I: 0,1 - Rumex arifolius All. I:0.1 - Peucedaum ostruthium Koch I: 0,1 - Saxifraga rotundifolia $\mathrm{I}$. 1;0.1 - Epilobium trigonum L. $I ; 0,1$.

Fagion et Fagetalia silvaticae.

Actaea spicata I. IV; 0,3 - Prenanthes purpurea I. IV: 0,2 - Paris quadrifolia 1 . III;0,3 - Asperula odorata 1 .. III 0.3 - Milium offusum L. II:0.2 - Myosotis silatica Hoffm. II;0.2 - Galeohdolon luteum Huds. II: 0.1 - Phyteuma spicatum L. ssp. ochroleucum Schulze II ; 0,2 - Fesfuca silaratica I. II ; 0,2 - Lilium martagon I. II : 0,1 - Corydalis solida $\mathrm{Sm} . \mathrm{N}: 0,5$ - Impatiens noli-tangre 1. . I; 0,1 - Euphorbia amygdaloides I. I; 0,1 .

Quercion et Quercetalia robori-sessiliflorae.

Salidago virga-aurea $\mathrm{L}$.. I; 0,1 - Maianthemum bifolium D.C. $\mathrm{I} ; 0.1$ Orobus tuberosus I. I; 0,1 - Deschampsia flexwosa Griseb. I ; 0,1

Querceto-Fagctea.

Anemone nemorosa L. II 0.3 - Melica tmiflora Retz II 0.1 - Cirsium erisithales L. II; 0,1 - Bromus asper L. I: 0,1 - Viola silvestris Koch $\mathrm{I} ; 0,1$.

Especes compagnes.

Oxalis acetosella L. III ; 0,5 - Sambucus racemosa L. III ; 0,3 Sorbus aucuparia L. (germinations) II ; 0,3 - Sorbus aucuparia L. 
(arbustif) II; 0,1 - L.uzula maxima L. II; 0,1 - Carex muricata L. II $; 0,1$ - .... (18 relevés).

Carex leporina L., commun dans les prairies environnantes, s'infiltre à l'intérieur de la hêtraie à la faveur de conditions écologiques favorables. Il revẻt alors souvent la forme argyroglochin Koch qui n'est guère qu'un acconmodat. Aspidium aculeatum Sw. ssp. angulare Kit. est une silvatique ombrophile à sa limite supérieure, ce n'est qu'une caractéristique locale. Polystichum spinulosum D,C. ssp. cu-spinulosum Mill, s'élève le long des bords humides des ruisseaux.

Athyrium filix-foemina Roth. qui a servi à dénommer notre sousassociation est très fréquente et simultanément doninante. Ce n'est pourtant que statistiquement qu'il est possible d'en faire une caractéristique car on la rencontre dans le Fageto-Scilletum typique, La méthode de la différentielle statistique nous donne une valeur de $t$ de 3,42 alors que le $t$ de STUdent-Fischer pour une probabilité de $95 \%$ est de 2,03. Il en est de même de Melandryum silvestre Roehl. ( $\mathrm{t}$ calculé $=3,66$ et $\mathrm{t}=2,01$ ) et de Dryopteris filix-mas Schott. ( $\mathrm{t}$ calcule $=4,48$ et $\mathrm{t}=2,05$ ). Sur le terrain, on reconnait d'un coup d'cil la sous-association et l'association; la méthode de la différentielle statistique faisant appel à $\overrightarrow{\mathrm{x}}$ se révèle beaucoup plus précise et exacte que la simple considération de la présence ou de l'absence des espèces, généralement employée.

Dans les Monts Dores, cette sous-association est bien développéc et présente sur toute l'aire de la hêtraie. Cependant elle est souvent fragmentaire comme nous l'avons déjả signalé. Elle nous a paru bien typique dans les Monts du Cantal, notamment dans la vallée de l'Allagnon. Une sous-association très semblable, mais privée de Scilla lilio-hyacinthus se rencontre dans les Monts du Forez. Nous ignorons son extension sur le glacis ouest du Massif Central. Il est probable qu'elle $y$ existe.

\section{3 - Le Fageto-Scilletum equisetosum.}

Nous avons élevé au rang de sous-association un aspect des hètraies des Dores. II se caractérise à première vue par sa richesse en Prêles. Sous une strate arborescente où les hêtres sont exclusifs, la strate arbustive est trés pature et pratiquement nulle. Par contre, la strate herbacée montre un recouvrement moyen de $80 \%$, avec une dominance nette d'Equisetum hyemale L. qui est parfois en peuplements presque purs, (II s'agit évidemment de la race occidentale à cótes biangulaires).

La fréquence la plus grande de cette association se trouve pour une altitude optimum assez basse, de l'ordre de $1000 \mathrm{~m}$. En effet. elle exige la présence du hètre et des prẻles, qui ont des réparti- 
tions altitudinales différentes - les prêles sont rares au-dessus de $1100 \mathrm{~m}$. I a pente du sol est de $35^{\circ}$ en moyenne. Les prèles poussent done sur un sol horizontal qui n'est pas inondé en permanence. L'exposition nord-est semble préférentielle et on peut penser qu'elle est en rapport avec les conditions écologiques que préfère le groupenent.

L'homogénéité statistique de la susts-association, fournie par le coefficient de Sokensex, est plus faible que dans les cas précédents. Cela sexplique par la relative abondance d'especes prairiales apportées par les eatux.

Caractíristiques de sous-association.

Equisetum hyemale L. V: 3.3 - Calanagrostis lanceolata Roth. II 0,5 .

\section{Caractéristiques de l'assoriation.}

Fagus silvatica L. V; 3,3 - Scilla lilio-hyacimhus L. III; 0,4 - Circaea intermedia Ehrh. III; 0,2 - Euphorbia hyberna L. II; 0,3 Stellaria nemorum L. ssp. glochidisperma Murb. I; 0,1 - Fagus silvatica L. (arbustif) I: 0,1 - Fagus silvatica L. (germinations) I; 0,1 - Pulmonaria affinis Jord. ssp. cu-affinis P.F. I; 0,1 - Epilobium nontanum I. I; 0,1 ,

Fagion et Fagetalia silvatirae.

Myosotis silatica Hoffm. III; 0,4 - Impatiens noli-tangere L. III ; 0,3 - Asperula odorata L. II: 0,2 - Phyteuma spicatum L. ssp. ochroleucum Schulze II:0.2 - Festuca heterophylla Lam. II 0.2 Scrophularia nodosa I. II; 0.2 - Daphne mezereum I. I: 0.1 - Paris quadrifolia 1. 1:0,1 - Cicerbita muralis Wallr. 1;0,1 - Poa nemoralis I. 1;0,1 - Veronica montana L. I; 0,1 - Festuca silatica Vill. $\mathrm{I} ; 0,1$.

\section{Querceto-Fagctea.}

Melandryum silvestre Roehl, 111; 0.3 - Viola silvestris Koch I: 0,1 - Anemone nentorosa $\mathrm{I}$. I I 0,1 .

\section{Sispèces compagnes.}

Ranunculus aconitifolius I. 1II: 0,3-Cardaniuc impatiens 1., III : 0,1 - Angelica silaestris I. II 0,2 - Dryopteris phegopteris Fée II ; 0,2 - Poa chaixii Vill. I; 0,1 - Avena pubescens I. I; 0,1 -Agrostis capillaris L. $1 ; 0,1$ - Phalaris arundinacea L. I; 0,1 - Lotus corniculatus L. I; 0,1 - Alchemilla vulgaris Rchb. I; 0,1 - Senecio doronicum L. I; 0,1 - Aconitum vulparia Rchb, I; 0,1 - Equisetum silvaticum L. I; 0,1 - Dryopteris filix-mas Roth. I; 0,1. 


\section{Muscinées.}

Mnium hormum I. I1;0,3 - Mmium undulatum Neck. I; 0,4-Hypnun cuspidatum L. 1;0,1 - Eurhynchirm sp. 1; 0,1. (11 relevés).

Les deux différentielles sont pratiquement exclusives de la sousassociation. Les stations étudiées n'avaient pas été signalées par les auteurs locaux précédents.

Dans les Monts Dores, la sous-association est relativenent rare et présente seulement dans les régions basses. Dans les Monts Dómes, elles semble également fragmentaire et passe au Querceto-Carpinetum montanum Lemék 1956, dans la vallée de la Pépinière près de Royat par exemple.

Dans le Forez, Legrand a signalé liquisetum hyemale dans une hêtratie au nord du Roc de Gourgon vers $1400 \mathrm{~m}$. SALANon (communication verbale) a retrouvé cette station et nous a confirmé qu'elle coincidait avec la sous-association des Dores. Dans le massif du Cantal elle semble présente dans les bois du Siniq et les bois de Chenuscles et d'Algére.

\section{4 - Le Fageto-Scilletum moehringietosum.}

II nous a été donné d'observer une sous-association assez peu répandue mais à caractères tranchés. Elle est liée à l'évolution naturelle des forẻts, résultant d'une diminution marquée du pourcentage de recouvrement de la strate supérieure, sans que cette diminution soit provoquée par des coupes. Elle peut donc résulter de chablis peu étendus ou encore de la chute d'arbres morts sur pied, qui se décomposent ensuite sur place. Cette sous-association est souvent fragmentaire conme on peut penser, mais nous avons pu observer une surface assez grande qu'elle recouvrait dans la forêt de Sarrevielle.

Comme il est normal, laltitude de cette sous-association est la mème que celle de l'association typique, La pente est également très comparable. La fréquence selon l'exposition n'est nullement préférentielle. Le substratum géologique est varié. Le recouvrement de la strate arborescente est anormalement faible, de l'ordre de $20 \%$ avec une strate arbustive faible également. Par contre, la strate herbacée est très exubérante et son degré de recouvrement atteint $100 \%$; parfois même il faudrait considérer deux strates herbacées superposées. Le coefficient de SokENsfx traduit une excellente homogénéité statistique.

\section{Différentielles de facies.}

Geranium robertianum L. IV ; 2,4 et $1 ; 0,1$ - Cardamine impatiens L. I; 0,1 et IV; 1,9 . 
Différentielles de sous-association.

Moehringia trinervia Clairv. V; 2,5 - Galeobdolon luteum Huds. $\mathrm{V} ; 2,5$ - Galeopsis tetrahit L. ssp. eu-tetrahit P.F. IV; 0,4 - Arum maculatum L. HI; 0,1 - Mercurialis perennis I. II ; 0,1.

Caractéristiques d'association.

Fagus silvatica L, V; 0,7 - Pulmonaria affinis Jord, ssp. eu-affinis P.F. V; 0,4 - Stellaria nemorum L. ssp. glochidisperma Murb. V; 0,4 - Fagus silvatica L. (germinations) IV; 1,9 - Scilla lilio-hyacinthus L. IV; 0,4 - Euphorbia hyberna L. IV; 0,4 - Conopodium denudatum Koch $1 \mathrm{~V} ; 0,3$ - Epilobium montanum L. III;0,6 - Fagus silvatica $\mathrm{I}$. (arbustif) $1 \mathrm{I} ; 0,3$ - Circaea intermedia Ehrh. II;0,2.

Fagion et Fagetalia silvaticae.

Myosotis silvatica Hoffm. V;0,3 - Asperula odorata L. IV; 0.3 Cicerbita muralis Wallr, III; 0,3 - Dentaria pinnata L.mk. III;0,2 Actaea spicata I. II; 0,1 - Paris quadrifolia L. 1II;0,1 - Prenanthes purpurea L. III ; 0,1 - Phytema spicatum L. ssp. ochroleucum Schulze II; 0,7 - Milium offusum I. II;0,7 - Poa nemoralis L. $\mathrm{II} ; 0,1$

\section{Querceto-Fagetea.}

Viola silvestris Koch IV; 0,5 - Melandryum silvestre Roehl. III ; 0,1 - Campanula trachelium L. II;0,2 - Melica uniflora Retz I; 0,1 - Cirsium erisithales Scopoli 1; 0,1 - Mex aquifolium I. I; 0,1 Anemone nemorosa 1. 1:0.1 -Lusula nivea D.C. I; 0,1 - Bromus asper $\mathrm{L}$., I ; 0,1.

\section{Espèces compagnes.}

Oxalis acetosella $\mathrm{L}, \mathrm{V} ; 0,5$ - Ranunculus aconitifolius $\mathrm{L} . \mathrm{V} ; 0,4$ Dryopteris filix-mas Schott. IV; 0,4 - Athyrium filix-foemina Roth. III; 0,4 - Valeriana officinalis L. (et ssp. div.) I; 0,1 Hieracium vulgatum Fries $1 ; 0,1$ - Urtica dioica L. I; 0,1 - Glechoma hederacea L. I; 0,1 - Senecio cacaliaster L.mk. I;0,1 - Ajuga reptans L. I; 0,1 - Galium uliginosum L. I; 0.1 - Poa chaixii Vill. 1;0,1 - Epilobium spicatum Lam. I;0,1.

\section{Muscinées.}

Polytrichum formosum Hedw. 11;0,3 - Orthotrichum sp. I; 0,2 Homalothecium sericeum Br. Fur. 1;0,1 - Mnium undulatum Neck. I : 0,1 - Mnim roscun Hedw, I; 0,1.

On peut considérer à l'intérieur du Fageto-Scilletum moehringietosum deux facies, l'un à Gcranium roberlianum et l'autre à Cardanine impatiens L. Ils se distinguent immédiatement sur le terrain par la nette dominance de ces espèces. 
Les différentielles de sous-association sont an nombre de cinq: deux sont exclusives à lintérieur des hètraies. sur notre territoire tout an moins. Arum maculatum et Mercurialis perennis, Moehringia trineria Clairveaux, présente dans l'association typique avec un faible degré de présence est très abondante dans cette sons-association. Elle nous a part rare en dehors, bien que Cinssagne la signale " très commune dans les Monts Dores " dans son Inventaire analytique. De mène Galcobdolon luteun ne se trouve guère que dans la sous-issociation. Ia méthode de la diffétentielle statistique nous donne $t$ calculé $-6,08$ (pour $t=2,019$ ). Elle est donc caractéristique de façon hautement significative, Galeopsis tetrahit est une espece d'endroits bien éclairés clans les sous-bois, il n'est pas étonnant de la rencontrer ici. L.es deux différentielles de facies sont également différentielles de la sous-association par rapport à l'association typique, de façon hautement significative.

Dans les Dores cette sous-association est assez rare car liée, strictement semble-t-il, à l'absence d'intervention de l'homme. C'est dans les hétraies les plus a sauvages " celle de Sarreviclle par exemple, qu'elle est le mieux représentée. Elle n’en semble pas moins devoir revètir une grande importance en ce qui concerne l'évolution naturelle de la végétation. Dans les Monts Dòmes, encore plus exploités et dégradés que les Dores, nous en avons vu un seul individu d'association, dans les bois de Volvic. Dans le Forez une variante appauvrie parait se rencontrer sur le versant dominant la plaine de Montbrison. Au sud elle atteint les environs de Saint-Flour. Nous ignorons sa limite ouest: elle existe aux environs de Messeix (de Lachapelle - communication verbale).

\section{5 - Le Fageto-Scilletum allietosum.}

Une autre sous-association est bien caractérisée dans les parties les plus élevées de nos forèts. Allimm victorialis $\mathrm{L}$. étant à lat fois dominante et différentielle nous l'avons intitulée Fageto-Scilletum allietosum en 1960. On peut la rencontrer sur des surfaces assez étendues, mais généralement d'accès peu facile.

Laltitude optimale de cette sous-association est la plus haute que nous ayons notée: $1400 \mathrm{~m}$ environ. La limite inférieure ne semble pas descendre bien en dessous de $1300 \mathrm{~m}$ et la limite supérieure est celle de la forêt, nous avons relevé un individu d'association à $1520 \mathrm{~m}$. La pente du sol est très forte et atteint cotramment $60^{\circ}$. ce qui est clù à la forme très redressée des reliefs érodés par les glaciers. Le substratum est varié, mais toujours volcanique. L'exposition est nettement préférentielle; la sous-association est pratiquement toujours localisée sur des pentes nord ou est et nous ne lavons pas rencontrée aux expositions ouest ni sud. L'étude des sols et de leur genèse nous permettra de l'expliquer. Physionomi- 
quement, la hètraie se présente comme une futaie avec quelques rejets de hẻtres en sous-bois. La strate herbacée est bien représentée, mais la strate muscinale très pauvre.

Différenticlles de sous-association.

Allimn victorialis $1 ., \mathrm{V} ; 1,2$ - Prenanthes purpurea L., V: 1,2 Calamagrostis armondinacea Roth. IV: 0,2 - Lilium martayon L. IV; 0.2 - Aspidium lobatum Sw, III: 0,1 - Pulmonaria affinis Jord. ssp, alpestris $1 . \mathrm{mk}$. III ; 0,1.

Caractéristiques d'association.

Faqus silvatica L. V:2.7 - Scillia hilo-hyacinthus L. V:0.5 - Euphorbia hyberna L. III; 0,3 - Pulmonaria affinis Jord. ssp. cll-affinis P.F. $111 ; 0,2$ - Fagus silvatica (germinations); 11 ; 0,5 - Conopodium denudatum Koch $\mathrm{II} ; 0,2$ - Circaea intermedia Ehrh. $\mathrm{II} ; 0,2$ Fagus silvatica L. (arbustif) 1: 0,1 - Polygonatum verticillatum All. $1: 0,1$.

\section{Fagion et Fagyctalia silaticae.}

Asperula odorata I. V: 0,7 - Phytema spicatum 1. ssp. ochroleucum Schulac IV : 0,5 - Paris quadritolia 1. III:0,1 - Galcohdolon lutcum Huds. II 0,2 - Actaea spicata I., II 0.3 - Stellaria nemorun I.. ssp. cu-nemorm Murb. II; 0,1 - Myosotis siliatica Hoffm. II: 0.1 - Epilobium duricui Gay 1: 0.1 - Dentaria pinnata D.C. I: 0,1 .

Quercion et quercetalia robori-sessiliflorac.

Hicracium groupe murorum III; 0,1 - Deschampsia flexuosa Griseh. II: 0,3 - Solidago sirga-aurea 1. II;0,1 - Orobus tuberosus I. 1:0,1 - Pirola minor 1. 1;0,1 - Veronica officinalis 1. 1:0,1.

Querceto-Fagetea.

Anemone nemorosa L. IV: 0,4 - Viola silaestris Koch IV:0,3 Melandryum silèestre Roeh1 $11: 0,2$ - Melica uniflora Retz $1: 0,3$ Bromus asper L. 1:0,1-Ranunculus breynimss Crantz 1; 0,1.

\section{Espices compagnes.}

Dryopteris filit-mas Schott, $\mathrm{V}: 0,7$ - Athyrium filix-focmina Roth. V: 0.6 - Ranunculus aconitifolits 1. II; 0,2 - Poa chairii Vill. II $; 0.2$ - Galium saratile $\mathrm{G}$. et $\mathrm{G}$. Weihe $\mathrm{I} ; 0.1$ - Oralis acetosella $\mathrm{L}$. 0,1 - Streptopus amplexifolius D.C. 1:0 - Sorbus aucuparia L. 1; 0.1 - Lusula maxima D.C. I: 0,1 - Lusula glabrata Desv. ssp. des*anxii Kunth $1 ; 0,1$ - Anthoxanthum odoratum L. 1:0,1 - Carcx polyrrhisa Wallr. (= C, umbrosa Hoppe) I: 0,1 - Nardus stricta L. $1 ; 0.1^{*}$.

* Nardus stricta, comme Anthosanthum odoratum a Carex polyrrhisa se sont infiltrés dans le sous-bois à partir des prairies environnantes. 
Muscinces.

Hylocomium triquetrum Br. Eur. II : 0.1 - Hylocomium splendens Br. Fur. I; 0.1 - Dicramum of. scoparium Hedw. I; 0,1. (16 relevés).

La sous-association comprend six différentielles par rapport à l'association type. Dryopteris lobata Schinz et Thell. (- Aspidium lobatum Sw.) et Pulmonaria affinis Jord. ssp. alpestre I amotte sont pratiquement exclusives de cette sous-association au sein du Fagion. en Auvergne tout au moins. Prenanthes purpurea L. est une différentielle statistique souvent dominante sur le terrain ( $\mathrm{t}$ calculé -3.9 pour $\mathrm{t}=2,01$ ). Calamagrostis arundinacca Roth. est tne espece caractéristique du Calamagrostidetum arundinaceae (de LACHAPELLE, 1961). C'est donc une caractéristique transgressive. La grande homogénéité du tableau de Czekasoswki nous confirme que la sous-association décrite n'est pas un simple terme de passage entre la hêtraie et la calamagrostidaic. Pour L.vouet, Alliam victorialis est une élective du Calamagrostidion; nous pensons, avec de LACHAPELLE qu'il s'agit plutót d'une espèce de la hètraie qui est transgressive dans la calamagrostidaie. Dans les Monts Dómes, LEMÉE (1956) considère Allium victorialis comme caractéristique dans le

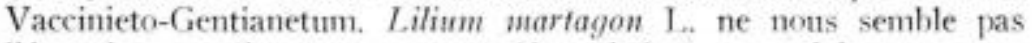
liée strictement à un groupement déterminé : on en a fait une caractéristique du Querceto-Carpinetum montanum et du Fraxino-Carpinion (LEMÉ, 1946), mais de LACHAPELLE (1961) la considère comme caractéristique du Calamagrostidetum. Il s'agit d'une espèce plus ou moins ombrophile, dont l'amplitude écologique est assez grande pour permettre sa végétation en sous-hois et dans les alpages à hautes herbes. A l'intérieur du Fagion. c'est une bonne différentielle locale.

Notons que nous avons rencontré le Fageto-Scilletum allietosum en des points où la forèt était éloignée du Calamagrostidetum, et passait en altitude à des nardaies.

Cette sous-association se rencontre en dehors des Dores. Fille ne semble néanmoins pás présente actuellement dans les Dônes; nous pensons que la reconstitution de la forèt de hêtres en certains lieux. occupés par les associations de dégradation du Brachypodietum et du Calluneto-Genistetum peut conduire à un groupement comparable après la mise en défens des puys. Dans le Forez et dans le Cantal, cette sous-assnciation se rencontre dans les hétraies du col des Supeyres (au-dessus de $1350 \mathrm{~m}$ ) et dans la vieille hêtraie de la vallée du Siniq.

\section{6 - Le Fageto-Luzuletum maximae.}

Nous avons écarté certains individus d'association du FagetoScilletum et de ses sous-associations pour en faire un groupement spécial que nous dénommons Fageto-Luzuletum maximae. Its se 
distinguent en effet par l'absence de Scilla lilio-hyacinthus et par la grande abondance des luzules, L.usula maxima étant dominante dans la strate herhacée. Remarquons dès à présent quielle n'est nas identique atu Luzulo-Fagetum de TExkx (1952) ni au Fagetun horeo-atlanticum luzuletosum de Marckgraf (1932).

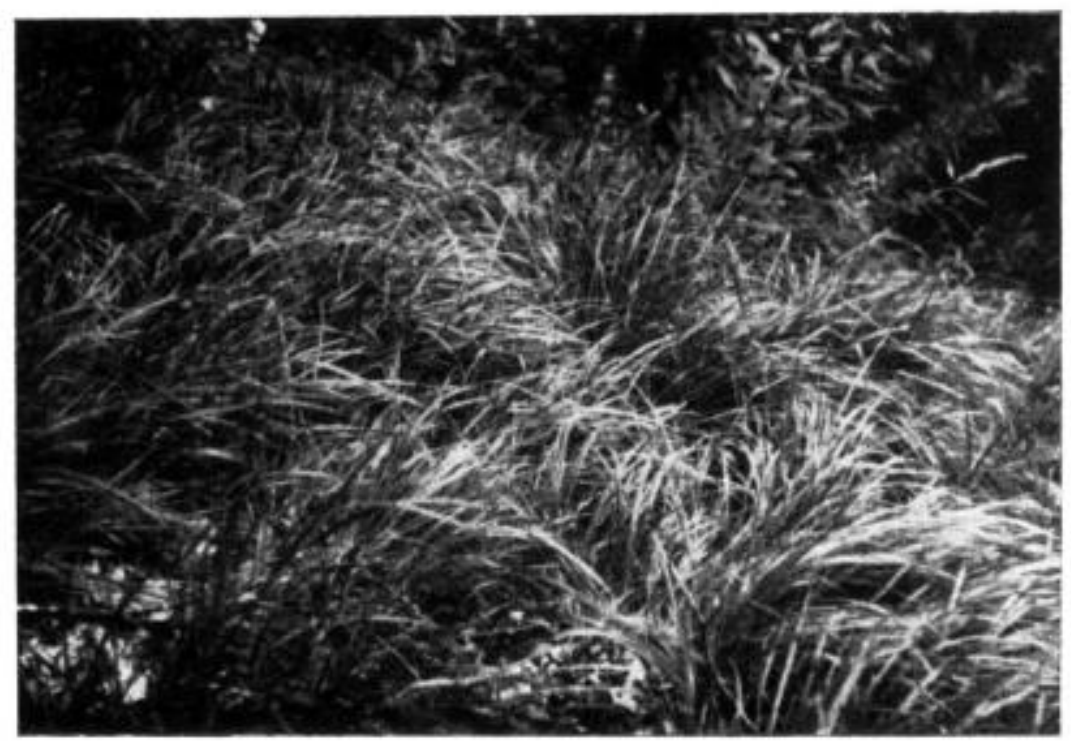

Sous-bois de Luzulo-Fagetum, Lusula sp, et Pod chairin.

(C.. Cusset.)

L'association se présente comme de belles futaies de hétres avec une strate arbustive très pauvre. Généralement l'exploitation semble légére, il semble néanmoins qu'elle pourrait être augmentée avantageusement. Nos relevés ont porté sur des individus d'associations où les arbres paraissent agés d'une cinquantaine d'années. Le Fageto-luzuletum existe dans la plupart des hétraies des Dores, de 800 à $1450 \mathrm{~m}$, et semble indifférent a la nature de la rochemere, crystallophyllienne, granitique ou volcanique. II marque une légére préférence pour l'exposition nord et nord-est.

\section{Caractéristiques d'association.}

Fagus silvatica L. V; 3,3 - Luzula maxima D.C. V : 1,6 - Poa chaitit Vill. IV : 0.6 - Festuca heterophylla Ldk. III 0.7 - Anemone ranunculoides L. II ; 0,3 - Daphne mezereum L. II 0.3 - Luzula pilosa Willd. I : 0,3 - Fagus silvatica L. (germinations) $1 ; 0,3$. 
Fagion et Fagetalia silvaticae,

Asperula odorata 1. III ; 0,3 - Euphorbia hyberna L. 11;0,4 Prenanthes purpurea L. II ; 0.2 - Phyteuma spicatum L. ssp. ochroleacum Schulze II ; 0,2 - Dentaria pinnata L. II ; 0,2 - Poa nemoralis L. II; 0,1 - Epilobium montanum L. I; 0,1 - Polygonatum verticillatum All. 1; 0,1 - Pulmonaria affinis Jord. ssp. eu-affinis P.F. I : 0,1 - Paris quadrifolia L. $1 ; 0,1$ - Actaea spicata L. I; 0,1.

\section{Quercion of Quercetalia robori-sessiliflorae.}

Solidago virga-aurea L. $111 ; 0,4$ - Sorbus aria Crantz $11 ; 0,4$ - Hieracium groupe murorum $\mathrm{II} ; 0,4$ - Orobus tuberosus L. II; 0,1 Veronica officinalis L. II ; 0,1 - Convallaria maialis L. I ; 0,1 - Melampyrum pratense L. I: 0.1 - Deschampsia flexwosa Griseb. 1; 0,1 .

\section{Querceto-Fagetea.}

Viola silvestris Koch III; 0,6 - Anemone nemorosa L. III; 02 Melandryum silvestre Roehl. II ; 0,1 - Ranunculus breyninus Crantz II ; 0,1 - Bromus asper L. I; 0,1 - lle, aquifolium L. I; 0,1 .

\section{Espèces compagnes.}

Oxalis acetosella L. IV; 0,5 - Senecio cacaliaster Lmk. II ; 0,3 Dryopteris filix-mas Schott. II; 0,3 - Silene inflata L. I; 0,1 Galium saratile G. et G. I: 0,1 - Ranunculıs aconitifolius L. I; 0,1 - Cardamine impatiens L. 1;0.1 - Knantia silvatica Duby I; 0.1 Lonicera nigra L. I; 0,1 - Carex muricata L. I ; 0,1.

\section{Muscinces.}

Hylocomium triquetrum Br. Eur. II ; 0,6 - Polytrichum formosum Hedv. 1; 0.5 - Hylocomium splendens Br. Eur. I; 0.1 - Dicranum scoparium Hedw. I; 0,1. (25 relevés).

Nous avons considéré deux faciès de cette association reconnaissable à la présence ou à labsence de peuplement de Luzula nivea D.C. Cette espèce, orophyte à exigences écologiques mal connues, est plutôt occidento-méridionale et il est possible que sa présence traduise un micro-climat légèrement plus chaud que celui du Fageto-Luzuletum typique. Lusula maxima D.C. est conmune en Auvergne de 450 à $1600 \mathrm{~m}$. Bien qu'elle ne soit pas exclusive de notre groupement, c'est elle que nous avons choisie pour le nom de notre association, car sa haute taille et sa vigueur lui font dominer les autres especes herbacées. Luzula pilosa Willd. est relativement fréquente dans les forèts d'Auvergne, c'est une différentielle à l'intérieur de la hètraie des Dores. Il en est de même d'Anemone ranunculoides, plante des lieux les plus humides du Querceto- 
Carpinetum montanum des Dômes. Lugover en avait cependant fait une caractéristique exclusive de son "association à Fagus silvatica ". Nous n'adoptons pas cette opinion. Poa chairii Vill, est une bonne différentielle statistique ( $\mathrm{t}$ calculé $=3,41$ pour $\mathrm{t}=2,01$ ) comme Festuca heterophylla Lam (t calculé - 3,36 pour $\mathrm{t}-2,03$ ).

L'aire de l'association nous parait aussi vaste que celle du FagetoScilletum dans les Dores, les Dómes et le Cantal, A l'est, elle nous semble s'étendre davantage et les bois du versant est du Forez en font probablement partie de façon générale (hêtraie de Gourgon, des Supeyres). A l'ouest, elle ne dépasse pats lat hêtraie des environs de Bourg-Iastic

Dans la vallée de la Dordogne on rencontre beaucoup plus fréquemment que dans les Dores her aquifolium en taillis sous la futaie. Il nous semble sagir lit diune variante du Fageto-I uzuletum tel que nous le comprenons et nous proposons d'appeler cet aspect de la hêtraie Fageto-l uzuletum ilicetosum. II caractérise les zones les plus atlantiques du Massif Central.

\section{B - Les hêtraies de l'ordre des Quercetalia}

Bien que le nom nème de Quercetalia évoque les bois de chènes tels que les Quercetum occidentale de BraUn-BLANQuet) ou les Querceto-holcetum mollis, il faut remarquer que $\alpha$ forêts de hêtreet association du hètre ne sont pas nécessairement des termes équivalents n (Brsus-Bunnouet). Nous justifierons dans la cinquième partie de ce mémoire la position systématique des hétraies que nous allons décrire.

\section{1 - Le Fageto-Deschampsietum flexuosae Lemée 1959.}

Cette association a été définie dans la légende de la carte phytogéographique des Monts Dómes par Lexík en 1959. Bien que, à notre connaissance il n'ait pas été publiè de tableau de l'association, il ne fait aucun doute que nos individus dassociation appartiennent à ce groupement.

L'altitude optimum de ce groupenent daus les Dores est d'environ $1300 \mathrm{~m}$ mais nous l'avons rencontrée de 1000 à $1400 \mathrm{~m}$; la pente est forte, autour de $40^{\circ}$ et le substratum varié. Les expositions nord, nord-est et nord-ouest sont nettement les plus fréquentes. Les arbres sont généralement bien vents mais lés germinations sont bien plus rares que dans les associations vues précédemment.

Nous avons distingué, outre P’association typique, un faciès à Hypnacées. Dans l'association typique les arbres n'ont qu'un recouvrenrent-d'environ $60 \%$-en moyenne: La strate muscinale n'est 
pas absente du facies typique et seule l'étendue du tableau de CzEKANoWSKI nous a permis de ranger de façon sure nos individus d'association dans la bonne catégorie". L'homogénéité est meilleure dans le facies à Hypnacées mais le coefficient de SOREnsen reste constamment au-dessus de $26 \%$ et est très généralement supérieur à $30 \%$.

\section{Différentielles de facies.}

Hylocomium triquetrum Br. Eur. IV : 0,4 et II ; 0.1 - Hylocomium splendens Br. Eur. 11;0,3 et 1:0,1 - Hyputm cupressiforme (ssp. div.) II; 0,1 et $1 ; 0,1$.

\section{Caractiristiques d'association.}

Fagus silatica L. V: 2,6 - Deschampsia flexwosa Griseb, V: 2,2 Hieracium groupe murorum V:0,8 - Maianthemum bifolium D.C. II : 0,1 - Pirola minor 1. II: 0,1 - Campanula rotundifolia L. I1: 0,1 .

\section{Quercion ef Quercetalia sessiliflorae.}

Solidago virga-aurea L. III : 0.2 - Veronica officinalis L. III ; 0,2 Melampyrum silvaticum L. II; 0.3 - Orobus tuberosus L. II; 0.2 Teucrium scorodonia L. II : 0,1 - Laserpitiun latifolium L. I: 0,1 Melampyrum pratense L. 1; 0,1 - Hieracium vulgatum Fries 1: 0,1 - Heracium tridentatum Fries I; 0,1 - Sorbus aria Crantz I: 0,1 - Cephalantera ensifolia Rich. 1:0.1 - Trifolium medium 1. 1: 0.1 - Hypericum pulchrum I. I: 0.1 .

\section{Fagion et Fagetalia Silvaticae.}

Prenanthes purpurea L. II ; 0,4 - Asperwla odorata L. I; 0,3 - Phyteuma spicatum L. ssp. ochroleucum Schulze I; 0,1 - Lilium martagon L. I: 0.1 - Dentaria pinnata L. I: 0,1 - Poa nemoralis L. I $: 0,1$.

\section{Querceto-Fagetea.}

Anemone nemorosa L. III : 0,3 - Luzula nivea D.C. II : 0,2 - Viola silvestris Koch II ; 0,2 - Melandryum silvestre Roehl. I; 0,1 - Bromus asper L. I; 0,1 - Stellaria nemorum L. ssp. en-nemorum P.F. I: 0,1 - Ranunculus breyninus Crantz I; 0.1 .

\section{Espèces compagnes.}

Luzula maxima D.C. III ; 0,2 - Oxalis acetosella L. II; 0,1 - Anthoxantum odoratum L. III;0,1 - Calamagrostis arundinacea Roth.

- Rappelons que les tableaux de Czekaxowski sont intégrés au mémoire déposé au laboratoire de Botanique de Clermont-Ferrand. 
11: 0,1 - Galium saratile G. et G, 11; 0,1 - Genista pilosa L. I; 0,1 - Senecio cacaliaster Lam. II ; 0,1 - Poa chairii Vill. I: 0,1 -Jasione peremnis L. I: 0.1 - Holcus mollis L. I 0.1 - Betonica officinalis Trevisan 1:0.1 - Serratula tinctoria L. I; 0.1 - Lotus corniculatus L, $1 ; 0,1$.

\section{Muscineses.}

Polytrichum formosum Hedw. 11;0,1 - Dicranum scoparium Hedw. 1: 0,1 - Diphyscium sessile Lindb. 1; 0,1.

Cette association se caractérise par six especes a tendances acidiphiles. Deschampsia flexuosa Griseb, que lon rencontre dans le Genisto-Vaccinion des Dores, n'est pas rare en Auvergne en dehors de la Limagne marno-calcaire. Hieracium murorum est très commun spécialement sous la forme de $H$. silvularum Sudre; on rencontre aussi $H$. cxotericum Sudre et $H$. aegocladum 7.ahn, Hieracium nemorense Zahn est plus rare, Maianthemum bifolium D.C. est exclusive de l'association dans les Monts Dores. Pirola minor L. est légèrenent différente des exemplaires typiques (sépales plus longs que larges, style plus long que l'ovaire). Campanula rotundifolia L. espèce très polymorphe est représentée par au moins deux variétés, scopulicola Lamotte et montana Lecoq et Lamotte; il s'agit d'une micro-endémique en évolution.

Le Fageto-Deschampsietum est courant dans les hêtraies des Dores et il s'y présente en général en surfaces assez étendues. Dans les Dómes l'état de dégradation de la végétation permet difficilement de le retrotver: nous sommes cependant convaincus que le Calluneto-Genistetum et surtout si sons-association Vaccinietosum LEAÉE 1956 l'a remplacé en bien des endroits. Dans le Forez, la hétraie du mont Sinêtre et celles du Montoncel en font partie. Elle ne semble pas rare sur le versint est qui domine la plaine de Montbrison. Dans le Cantal. nous l'avons également rencontrée. Nous ignorons son extension at dela des bois du Domais, sur le versant onest des Dores. Les conditions écologiques doivent lui convenir.

\section{2 - Le Fageto-Deschampsietum hylocomietosum.}

Au prenier abord, cette sous-atssociation ne differe pas nettement du facies a Hypnacées de l'association typique. Elle possède cependant une différentielle exclusive et les mousses $y$ sont des différentielles statistiques hautement significatives.

Il s'agit d'une futaie ì maigre strate herbacée (le degré de couverture ne défasse pas $30 \%$ ) oủ les arbres sont bien développés, mais où les germinations sont très rares, L'exposition nord est la plus fréquente et les exposition ensoleillées peu fréquentes.

Le tableau de Czekaxowski montre une homogénéité toujours supérieure à $30 \%$. 
Différentielles de sous-association.

Hylocomium splendens Br. Eur. V; 0,8 - Hylocomium triquetrum Br. Eur. V; 1,7 - Pirola secunda L. III ; 0,5.

\section{Caractéristiques d'association.}

Fagus silvatica L. V; 3,4 - Deschampsia flexwosa Griseb. V; 0,4Hieracium groupe murorum L. IV; 0,5 - Pirola minor L. II; 0,3 Maianthemum bifolium D.C. II ; 0.1 - Campanula rotundifolia L. I; 0.1 .

\section{Quercion et Quercetalia sessiliflorae,}

Solidago virga-aurea L. III : 0.5 - Melampyrum pratense L. III : 0,2 - Veronica officinalis L. III ; 0,1 - Tettcrium scorodonia L. I: 0,1 - Melampyrum silvaticum L. I: 0,1 .

\section{Fagion et Fagetalia silvaticae.}

Prenanthes purpurea L. 1; 0,1 - Phyteuma spicatum L. ssp. ochroleucum Schulze I ; 0,1 - Poa nemoralis L. 1: 0.1 - Cicerbita muralis Wallr. I; 0,1 .

\section{Querceto-Fagetea,}

Inemone nemorosa L. 11:0,3 - Viola silvestris Koch 11; 0,1 Stellaria holostea $1 ; 0,1$.

\section{Espèces compagnes.}

Luzula maxima D.C. IV; 0,4 - Juniperus communis III; 0,1 Oxalis acetosella $\mathrm{L}$. III; 0,1 - Sorbus aucuparia $\mathrm{L}$. II; 0,1 - Poa chaixii V. II : 0,1 - Lonicera nigra L. I; 0,1 - Potentilla erecta Garcke - Galim saxatile G. et G. I; 0,1.

\section{Muscinées.}

Hypnum cupressitorme L. II ; 0,3 - Polytrichum formosum Hedw. II ; 0,1 - Dicranum scoparium Hedw, I; 0,1.

(17 relevés).

On pourrait caractériser cette sous-association par Pirola secunda qui est une différentielle exclusive à l'intérieur des hètraies des Dores. Néanmoins Pirola secunda est souvent considérée comme appartenant à l'ordre des Vaccinio-Piceetalia et nous avons préféré utiliser Hylocomium splendens. Hylocomium splendens est nettement différentielle par rapport au facies à hypnacées du FagetoDeschampsietum typique ( $\mathrm{t}$ calculé $=2,389$ pour $\mathrm{t}-2,056$ pour 
une probabilité de $95 \%$ ). 11 en est de méme de Hylocomiun triquétrum ( $\mathrm{t}$ calculé $-2,077$ pout $\mathrm{t}-2,055$ pour une probalilité de $95 \%)$.

Cette sous-association est assez rare sous la forme typique de futaie de hêtres dans notre dition: elle est plus abondante sur le versant ouest des Dores, notamment dans la vallée de la Dordogne. Dans le Cantal, on la rencontre au moins dans la Hêtraie du Siniq. Elle semble se rencontrer dans les bois des environs du Brugeron dans le Forez.

\section{3 - Le Fageto-Deschampsietum vaccinietosum.}

Sous une maigre futaie de hêtres rabougris une strate arbustive de Vaccinium et de Callune est bien développée. La strate arborescente a un degré de recouvrement de $30 \%$ alors que la strate arbustive atteint $55 \%$. La régénération du hêtre est nulle; nous n'avons rencontré aucun semis naturel qui ait des chances de n’être pas étuuffé par la callune ou les myrtilles.

L'altitude est la même que celle du Fageto-Scilletum, de 1100 a $1400 \mathrm{~m}$ et la roche-mère ne semble pas avoir d'influence marquée sur l'association. L'exposition sud-ouest est, ste loin, la plus fréquente. Nous verrons que cest, en effet, celle du maximum de lessivage des sols, correspondant aux pluies dominantes.

Différentielles de sous-association.

l'accinium myrtillus L. V; 2,3 - Calluna vulgaris Salibs. III;0,4 Lencobryum glaucum Hamp. $1 \mathrm{II} ; 0.4$ - Pteridium aquilinum Kuhn III $; 0.1$.

\section{Caractíristiques d'association.}

Fagus silvatica L. V; 1,3 - Hicracium groupe murorum L. IV ;0,3 Deschampsia flcxuosa Griseb. III ; 0,3-Pirola minor L. III ; 0,1 Maianthemm bifolium D.C. $11 ; 0,1$.

Quercion at Quercetalia robori-sessiliflorae.

Veronica officinalis L. V: 0,4 - Melampyrum pratense $\mathrm{L}$. $111 ; 0,3$ Solidago virga-aurea L. III ; 0,2 - Orobus tuberosus L. III; 0,1 Sorbus aria Crantz III; 0,1 - Melampyrum silvaticum L. III : 0,1 Conzullaria maialis L. I; 0,1 .

Fagion et Fagetalia silvaticae.

Prenanthes purpurea L. $\mathrm{H} ; 0,1$. 


\section{Querceto-Fagetea.}

Yiola silvestris Koch $\mathrm{III} ; 0,3$ - Lusula nizea D.C. II $; 0,3$ - Abies pectinata Lmk. I; 0,1 - Ilex aquifolim L. 1; 0,1.

\section{Espèces compagnes.}

Lusula maxima D.C. III ; 0,5 - Oxalis acetosella L. III ; 0,1 - Holcus mollis L. II ; 0,1 - Betonica officinalis Trevis. II ; 0,1 - Calanagrostis arundinacea Roth. I; 0,1 - Sarothamnus scoparius Koch $1 ; 0,1$ - Blechnum spicant Withg. I;0,1 - Athyrium filir-focmina Roth. I; 0,1.

\section{Muscinées.}

Hylocomium triquetrum $\mathrm{Br}$. Lur. $11 \mathrm{H} ; 0,3$ - Hylocomium splendens Br. Eur. II ; 0,3 - Hylocomium loreum Br. Eur. II ; 0,1 - Hypnum cupressiforme L. 11;0,1 - Polytrichum formosum Hedw. I; 0,1 Dicranum scoparitm Hedw. I; 0,1

(16 relevés).

La sous-association se distingue par quatre différentielles: $\mathrm{Vac}$ cinium myrtillus L. qui est une espèce commune dans le Massif Central entre 900 et $1700 \mathrm{~m}$ est une différentielle à l'intérieur de la hêtraie, Calluna vulgaris Salisb. transgressive du Calluneto-Genistetum TüxEN 1937, qui est fréquent au-dessus des hêtraies, est une bonne caractéristique de la sous-association ( $\mathrm{t}$ calculé $=2,162$ pour $\mathrm{t}=2,019)$. Pteridium aquilinum Kuhn se rencontre uniquement dans les individus d'association les plus bas et disparait atdessus de $1300 \mathrm{~m}$. Leucobryum glaucum Hamp. est une mousse acidiphile qui fructifie rarement surtout à la limite supérieure de la sous-association. On la considère généralement comme caractéristique du Quercion sessiliflorae.

Cette sous-association, bien développée dans les Monts Dores semble absente des Dômes où elle est remplacée par des associations préférant une altitude moins élevée. Dans le Forez, par contre, et le Livradois, elle est très répandue (hêtraie de la Faye, hêtraie du Montoncel, bois des Gardes...). Elle est moins répandue dans le Cantal, bien qu'on la rencontre dans les hetraies de Marcenat.

\section{C - Le milieu de la hêtraie}

Après une étude statique des associations des Hètraies, nous allons entreprendre l'étude des conditions écologiques dans lesquelles elles vivent, examinant successivement les facteurs climatiques, édaphiques et biotiques. 


\section{1 - Les facteurs climatiques.}

Dans son ouvrage sur l'Indochine (1934), CARTon a proposé une classification des climats. Elle permet d'avoir une idée fort juste du climat moyen de nos foréts; Besse se range dans la zone deux. tempérée, et dans le grotupe $\mathrm{B}_{2}$ tempété à hivers froids. Le degré de continentalisme fourni par la formule de Gokczinski $\left(\mathrm{k}-\frac{1,7 \mathrm{~A}}{\sin \mathrm{L}}-20,4\right)$ est égal à 17,1 , soit * nettement uniforme ou marin ". Nous avons effectivement en altitude une saison seche estivale, caractère océanique.

L'indice " classique " le plus ancien est celui de LAxG; e'est simplement le rapport de la pluie à la température. $\Lambda$ Besse, il est égal à 159 et à Chaudefour il doit atteindre 300 .

Le coefficient de MEYÉr tient compte du déficit de saturation de T'air. A Besse, il est sensiblement égal à 970 car le déficit de saturation est de l'ordre de 1,2 (en mm de mercure), ce qui correspond à la zone des sols a faiblement podzolique $n$.

$$
\text { L.'indice de DE. Martonse } \mathrm{Ar}=\frac{\mathrm{P}}{\mathrm{T}+10} \text { fournit une valeur de }
$$

68 à Besse et d'environ 110 a Chaudefour: ce sont des valeurs de climat montagnard froid et humide.

Les courbes de Giaussen nous montrent enfin qu'il n'existe pas de saison seche a Besse. I.a courbe 4 T elle-même ne recoupe pas la courbe des pluies.

En résumé, tous ces indices climatiques, malgré leur variété, s'accordent pour reconnaitre à notre région un climat très humide et plutót froid. I a végétation ne souffre jamais de la sécheresse et le lessivage des sols peut être très important.

\section{a) Les temperatures,}

Nous avons noté les températures de l'air et du sol dans les différentes sortes de hêtraies pour comprendre l'influence de la végétation sur le microclimat.

Nous n'avons pas de mesures pour le Fageto-Scilletum a Ripuisetum. Les autres mesures ont été faites alors que le ciel était uniformément bleu et le vent très faible.

Les fougères épaisses dans le Fagetum-Scilletum athyrietosum abaissent la température au niveau de la strate inférieure. Cette diminution de la température est évidemment plus sensible en été oú les frondes sont développées, mais ces dernières persistent long- 
temps à larrière-saison et cet effet se fait sentir tant que la neige ne recouvre pas le sol.

Le Fageto-Scilletum allietosum marque une plus grande amplitude de variation due probablement à l'altitude élevée de cette sousassociation, où le vent est tantôt fort tantôt faible avec des sautes capricieuses rapides.

\begin{tabular}{|c|c|c|c|c|}
\hline & \multicolumn{4}{|c|}{ Phase de lumière } \\
\hline & $\begin{array}{c}150 \mathrm{~cm} \\
-\end{array}$ & $5 \mathrm{~cm}$ & $0 \mathrm{~cm}$ & -10 \\
\hline Fageto-Scilletum athyrietosum ...... & +0.5 & 0 & $-1,5$ & \\
\hline Fageto-Scilletum allietnstum ........ & +0.5 & +1.5 & $+2,5$ & +1 \\
\hline Fageto-Scilletum typique .......... & $+0,5$ & +0.5 & +1 & $+0,5$ \\
\hline Fageto-Scilletum moehringietosum .. & 0 & $+0,5$ & -0.5 & 0 \\
\hline Fageto-Luzuletum ................ & 0 & $+0,5$ & +1 & +1 \\
\hline Fageto-Deschampsietum $\quad \ldots . . . . .$. & +0.5 & +1 & $+1,5$ & +2 \\
\hline Fageto-Deschampsietum hylocomict. & $-0,5$ & 0 & $-0,5$ & +1 \\
\hline Fageto-Deschampsictum vaccin. .... & 0 & +1.5 & $+2,5$ & +2 \\
\hline
\end{tabular}

Phace d'ombre

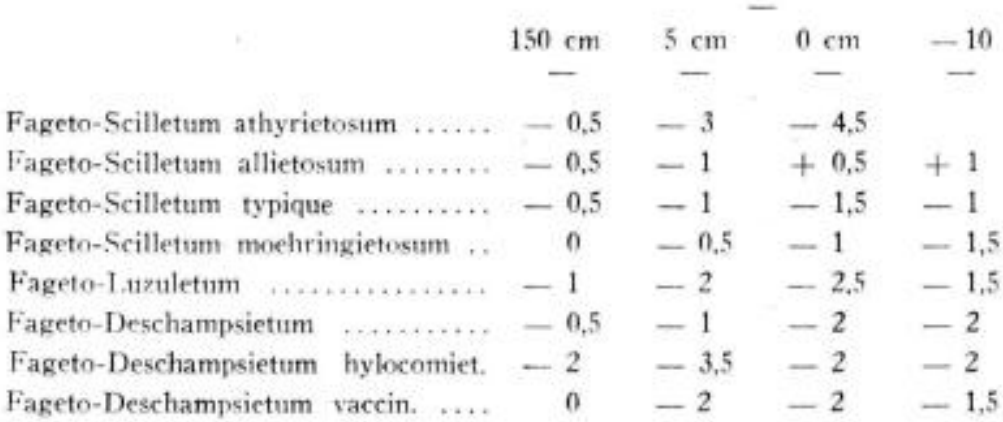

(Températures notées par rapport à celle d'une prairie)

Le Fageto-Scilletum moehringietosum a des températures bien voisines de celles des prairies environnantes, sauf au niveau du sol. En sont cause le faible degré de recouvrement de la strate arborescente et la strate herbacée très dense au contraire.

Par contre, la température du Fageto-Deschampsietum hylocomietosum est notablement abaissée, surtout en phase d'ombre par l'énais-

- La prairie de référence était bien entendu à la méme altitude et à la mème exposition. Les arbres étaient pourvus de leurs feuilles en phase d'ombre, et dépourvus en phase de lumière. 
se couverture de la strate arborescente. Le Fageto-Deschampsietum vaccinietosum voit la température des différentes strates varier au niveau de la strate arbustive; en saison froide elle protège l'air et le sol des vents les plus froids et en saison chaude elle donne une ombre drue et froide arrêtant les rayons du soleil presque totalement.

Les trois associations typiques, qui totutes trois se présentent conume des futaies à sous-bois peu encombré, montrent un faible abaissement en phase d"ombre et un léger réchauffement en phase de lumière. L'action des feuilles qui diminuent l'insolation estivale explique le premier, et le second est dù à la protection des arbres en saison froide. Les différences sont plus sensibles au ras du sol qu'en altitude oì ces actions sont moins marquées.

\section{b) La luminosité.}

Là aussi nous n'avons pu faire que quelques mesures comparatives par rapport à la luminosité à un mètre du sol dans des associations prairiales voisines (nardaies ou centaureo-cynosuretum).

La pente généralement forte de nos groupements augmente la litminosité et les chiffres que nous avons obtenus sont souvent supérieurs à ceux des autres auteurs. Précisons que nous avons tenu nos instruments non verticalement, mais en tenant compte de l'inclinaison du sol, condition à laquelle les plantes sont soumises. WiFsNER (1907) donnait une intensité lumineuse de 130,162 et 185 pour des pentes de 35,45 et $55^{\circ}$ contre tune intensité de 100 pour une surface horizontale.

I a même association présente naturellement des éclairements différents selon que l'on s'arlresse à des plages de lumière ou d'ombre sous le feuillage des arhres. Totutes nos mesures concernent des plages d'ombre. Nous avons noté en éclairement relatif:

\begin{tabular}{|c|c|c|}
\hline & $\begin{array}{c}150 \mathrm{~cm} \\
-\end{array}$ & $\begin{array}{c}5 \mathrm{~cm} \\
-\end{array}$ \\
\hline Fageto-Silletum typique & $4,5 \%$ & $3,5 \%$ \\
\hline Fageto-Scilletum moehringietosum. & $25 \%$ & $7 \%$ \\
\hline Fageto-Scilletum allietosum ................... & $6 \%$ & $2 \%$ \\
\hline Fageto-Scilletum athyrietosum . ................. & $6 \%$ & $3 \%$ \\
\hline Fageto-Luzuletum $\quad \ldots \ldots \ldots \ldots \ldots \ldots \ldots \ldots \ldots$ & $4 \%$ & $3.5 \%$ \\
\hline Fageto-Deschampsietum typique $\ldots . . . \ldots \ldots \ldots$ & $4 \%$ & $4 \%$ \\
\hline Fageto-Deschampsictum hylocomietostum .... & $3 \%$ & $2 \%$ \\
\hline Fageto-Deschampsietum vaccinietosum ..... & $9 \%$ & $3 \%$ \\
\hline
\end{tabular}

(100 \% = éclairement d'une prairie voisine).

On est immédiatement frappé par la très forte luminosité à $150 \mathrm{~cm}$ du sol du Fageto-Scilletum moeyringietosum. Il est évident que le faible pourcentage de recouvrement en est cause. 
Si l'on examine l'aptitude de la végétation à supporter l'ombre, dans le cadre de la classification de WIESNER, on voit que nos hétraies ne portent aucune plante "à la lumière obligatoire $n$ et que scule Vicio sepium (dans le F.S. moehringiesotum) appartient an groupe " héliophile ", Nardus stricta. Campanula rotundifolia, Vaccinium myrtillus et Pteridium aquilinum sont "indifférentes " at1 sens de Winsner. Remarquons qu'il s'agit surtout de plantes à nombreux écotypes et à amplitude écologique parfois considérable (Pteridium aquiliman se retrouve aussi bien dans les zones tropicales qu'au Groenland). Les autres plantes, c'est-à-dire presque toutes sont soit des "Umbrophiles " soit des a plantes craignant la lumière $n$.

Les seules observations suivies que nous ayons pu faire concernent la hètraie (Fageto-Scilletum) du Lac Pavin. Les résultats sont très comparables aux résultats classiques (voir par exemple QuANTIN, P. 253).

\section{c) L'humidité.}

Nous avons vu que l'humidité du méso-climat est trés forte. Fn est-il de mene pour les micro-climats?

Nos mesures d'évaporation, effectuées à l'évaporimètre de PrCHE (rondelles standard) sont fragmentaires. On en déduit néanmoins, de façon nette que l'évaporation augmente beaucoup des hẻtraies à fougères anx hêtraies à myrtilles. Toutes les mesures ont été faites à $20 \mathrm{~cm}$ du sol pendant deux heures.

\begin{tabular}{|c|c|}
\hline tum athyrietosum & \\
\hline Fageto-Scilletum typique. & 35 \\
\hline Fageto-I.uzuletum .. & 45 \\
\hline Fageto-Deschampsietum vaccinnietosum . & 70 \\
\hline Narklaie de référence & 100 \\
\hline
\end{tabular}

Le déficit de saturation est une des données les plus importantes pour caractériser l'état d'humidité de l'atmosphère. Voici les chiffres que nous avons obtenus:

Phase de lumière Phase d'ombre
$150 \mathrm{~cm} \quad 20 \mathrm{~cm} \quad 150 \mathrm{~cm} 20 \mathrm{~cm}$

Fageto-Scilletum athyrietosum $\ldots \ldots \ldots$.

\section{0}

Fageto-Scilletum allietosum .......... 95

Fageto-Scilletum typique ............. 95

Fageto-Scilletum moehringietosum .....

Fageto-Luzuletum .................. 95

Fageto-Deschampsietum ............. 100

Fageto-Deschampsietum hylocomietosum. 95

Fageto-Deschampsietum vaccinietosum ..

\begin{tabular}{ll}
- & \\
\cline { 3 - 3 } 90 & 20 \\
95 & 80 \\
95 & 8 \\
90 & 6 \\
95 & 9 \\
00 & 9 \\
95 & 85 \\
00 & 9
\end{tabular}

$\begin{array}{lll}20 & 60 & 15 \\ 80 & 75 & 55 \\ 85 & 75 & 60 \\ 60 & 70 & 45 \\ 90 & 80 & 65 \\ 90 & 90 & 70 \\ 85 & 90 & 85 \\ 90 & 95 & 90\end{array}$




\section{LFS FORETS DU VERSANT SUD DES MONTS DORES}

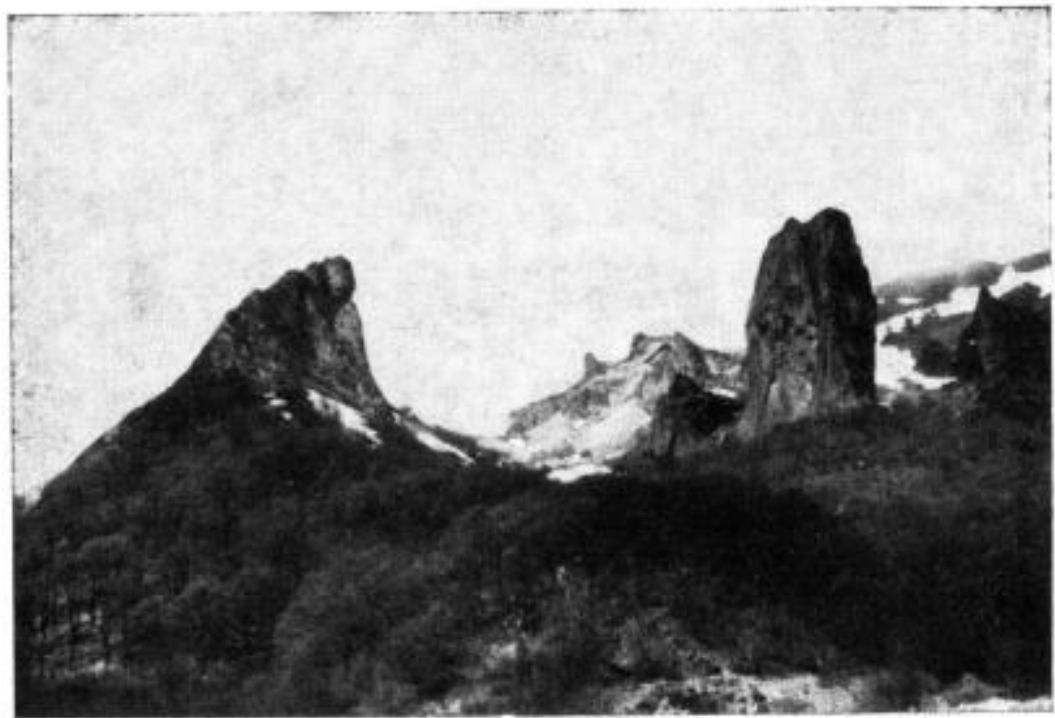

Hêtraie de la vallée de Chaudefour:

Dykes trachytiques dégagés par l'érosion.

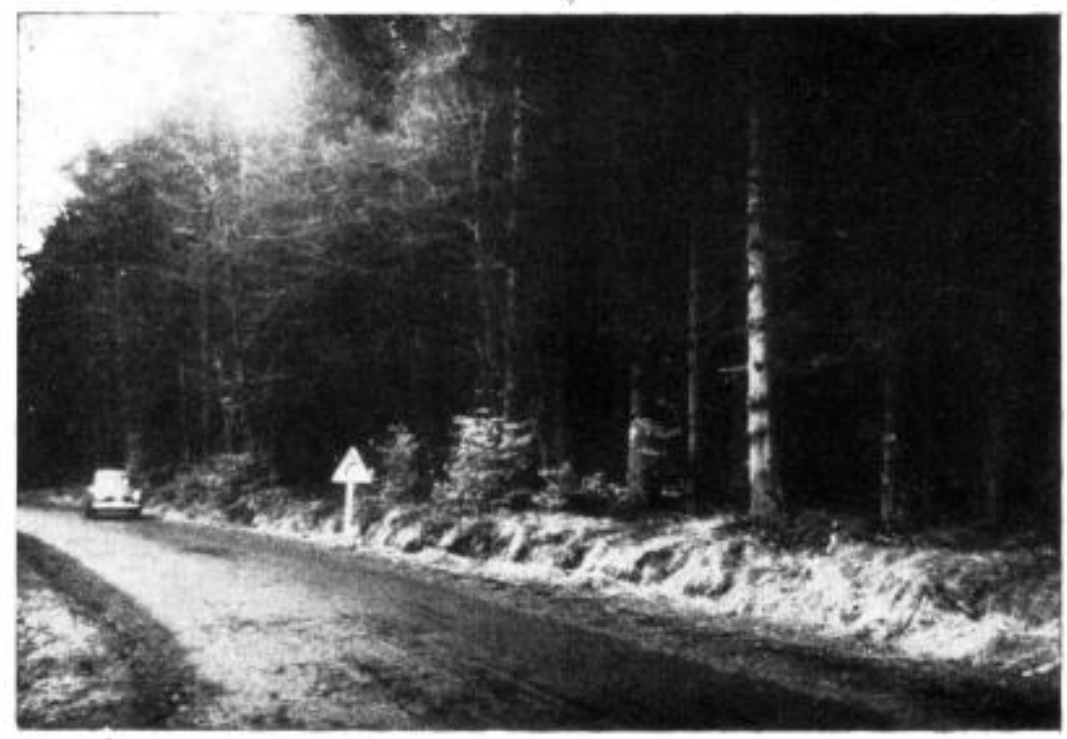

Forčt de Vaucoux, route de Besse

Régénération de hêtre dans le sous-bois de conifères, dès qu'apparait une éclaircie.

(Cl. Cusset.) 
Les variations du déficit de saturation sont parallèles à celles de l'évaporation. Par temps ensoleillé comme en période d'ombre, le déficit est supérieur au niveau du sol. LEMÉE avait des chiffres comparables dans les associations silvatiques mésophiles du Perche. Notons que les variations du déficit de saturation ont une amplitude moindre dans les associations les plus xérophiles, et que sa moyenne s'élève des groupements ombrophiles aux groupements xérophiles.

\section{2 - Les facteurs édaphiques.}

1.'étude des sols des hêtraies nous a permis de mettre en évidence une dizaine de types de sols. Nous commencerons par les moins évolués.

\section{i) Les Rankers à mull.}

L.e Fageto-Scilletum athyrietosum recouvre souvent des sols très peu évolués que l'on peut ranget dans la catégorie des rankers à mull. Un bel exemple recouvre une surface d'environ un hectare sur le versant sud de la vallée de Chaudefour.

Le sol est peu évoluć, à profil đu type $A_{1} C$. L'érosion continuelle a la lase des falaises apporte des éboulis, entraine les débris végétaux dès qu'ils recouvrent le sol, empéche un approfondissement. Les oxydes de fer sont libérés en partie, et colorent la partie superficielle en un brun sale. L'horizon humifère passe dès une dizaine de centimetres de profondeur à un horizon mixte, transition vers la roche-mère. I es racines des fougères atteignent et délitent cette roche-mère, souvent de nature cinéritique.

La composition granulométrique est donnée dans le tableat 2 où nous avons groupé les résultats des analyses de sols*.

\section{b) Le sol brun juvénile uniforme.}

La sous-association à fougères se rencontre également sur un sol un peu plus évolué que le précédent, un sol brun de profil pratiquement uniforme. L'analyse que nous donnons provient d'un échantillon prélevé dans la vallée de Chaudefour, sur une roche-mère de cinérites trachytiques.

D'assez nombreux blocs de trachyte arrachés par l'érosion à une falaise surplombante peuvent s'observer à la surface dı sol, et l'on pourrait être tenté d'en faire partie intégrante du sol. Cependant la végétation n'a de rapports qu'avec les parties du sol dénudées, les blocs, tant qu'ils ne sont pas emportés plus loin par la neige, ne jouant qu'un róle minime dans son déterminisme. Le profil est de type $\mathrm{A}(\mathrm{B}) \mathrm{C}$, sans lessivage horizontal sensible des colloides miné-

- Nous avons fait les analyses de sol en collaboration avec dE LACHAPELle. 
raux ni du fer. Ils sont entraines obliquement par l'eau courante et l'eau de fonte des neiges et ne se regroupent pas en un horizon différencié. L a grande porosité de la tuche-mère facilite ces déplacements. L'humus est un mull forestier à acidité faible, parfois neutre. Il existe une litière de feuilles mortes qui se décompose rapidement au printemps après une assez longue période hivernale où elle est recouverte par la neige. L'analyse montre un horizon (B) légèrement enrichi en collonles minéraux; il est praticuement indiscernable sur le terrain de la roche-mere, les traces ile

ANALYSE GIRANULOMETRIQUE DES SOIS DE HETRAIE

\begin{tabular}{|c|c|c|c|c|c|c|c|c|c|c|}
\hline & & $\mathrm{PH}$ & $\begin{array}{l}\text { gables } \\
\text { gross. }\end{array}$ & $\begin{array}{c}\text { sables } \\
\text { f1ns }\end{array}$ & s11ts & $\begin{array}{l}\operatorname{coll} . \\
\text { nin. }\end{array}$ & $\begin{array}{l}\text { coll. } \\
\text { hum. }\end{array}$ & $\begin{array}{c}\text { nat } 1 \text { er } \\
\text { org. }\end{array}$ & $\mathrm{CO}_{3} \mathrm{Ca}$ & éch. \\
\hline Aanker ì null & & 6,7 & 32,4 & 16,1 & 12,6 & 7,8 & 8,2 & $27, I$ & nul & 2,50 \\
\hline $\begin{array}{l}\text { Sol brun } \\
\text { forestier }\end{array}$ & $\left(\begin{array}{c}A_{1} \\
A_{2}\end{array}\right)$ & $\begin{array}{l}6,8 \\
6,9 \\
6,8\end{array}$ & $\begin{array}{l}22,5 \\
39,1 \\
27,8\end{array}$ & $\begin{array}{l}I 4,3 \\
25, I \\
2 I, 2\end{array}$ & $\begin{array}{l}19,1 \\
18, ? \\
24 ; 1\end{array}$ & $\begin{array}{l}6,7 \\
4,3 \\
9,2\end{array}$ & $\begin{array}{l}5,2 \\
2,4 \\
3,1\end{array}$ & $\begin{array}{r}30,6 \\
6,1 \\
14,2\end{array}$ & $\begin{array}{c}\text { nul } \\
\text { traces } \\
\text { traces }\end{array}$ & $\begin{array}{r}17,5 \\
8,7 \\
13,5\end{array}$ \\
\hline $\begin{array}{l}\text { Sol brun } \\
\text { un1forme }\end{array}$ & $\begin{array}{l}(\vec{A} T) \\
(B C)\end{array}$ & $\begin{array}{l}6,7 \\
6,8 \\
6,8\end{array}$ & $\begin{array}{l}36,8 \\
47, ? \\
54,5\end{array}$ & $\begin{array}{l}16,9 \\
26,0 \\
17,7\end{array}$ & $\begin{array}{r}5,6 \\
5,3 \\
15,1\end{array}$ & $\begin{array}{l}1,4 \\
2,8 \\
1,9\end{array}$ & $\begin{array}{c}3, ? \\
0,8 \\
\text { traces }\end{array}$ & $\begin{array}{r}31,2 \\
15,5 \\
6,8\end{array}$ & $\begin{array}{l}\text { nul } \\
\text { nul } \\
\text { traces }\end{array}$ & $\begin{array}{l}9,1 \\
3,8 \\
3,5\end{array}$ \\
\hline $\begin{array}{l}\text { Sol brun } \\
\text { lessivé }\end{array}$ & $\mathrm{B}^{\mathrm{A}_{\mathrm{I}}}$ & $\begin{array}{l}5,4 \\
5,6 \\
5,8\end{array}$ & $\begin{array}{l}29,2 \\
44,5 \\
31,5\end{array}$ & $\begin{array}{l}11,4 \\
22,1 \\
18,2\end{array}$ & $\begin{array}{l}12, ? \\
11,3 \\
14,1\end{array}$ & $\begin{array}{l}18,5 \\
12,3 \\
22,5\end{array}$ & $\begin{array}{l}6,2 \\
I, 2 \\
2,4\end{array}$ & $\begin{array}{r}20,5 \\
4,2 \\
3,1\end{array}$ & $\begin{array}{l}\text { nul } \\
\text { nul } \\
\text { nul }\end{array}$ & $\begin{array}{l}22,7 \\
10,4 \\
32,7\end{array}$ \\
\hline Parabraunerde & $\begin{array}{l}\hat{A}_{I} \\
A_{2}^{2}\end{array}$ & $\begin{array}{l}4,7 \\
5,7 \\
4,8\end{array}$ & $\begin{array}{l}27,1 \\
37,1 \\
31,0\end{array}$ & $\begin{array}{l}26,4 \\
24,3 \\
18,3\end{array}$ & $\begin{array}{l}18,6 \\
21,4 \\
24,0\end{array}$ & $\begin{array}{l}13,4 \\
I I, 4 \\
23,6\end{array}$ & $\begin{array}{r}5,1 \\
0,8 \\
\text { traces }\end{array}$ & $\begin{array}{c}12,4 \\
1,2 \\
\text { traces }\end{array}$ & $\begin{array}{l}\text { nul } \\
\text { nul } \\
\text { traces }\end{array}$ & $\begin{array}{r}8,2 \\
6,8 \\
13,8\end{array}$ \\
\hline $\begin{array}{l}\text { Sol brun } \\
\text { podzollque }\end{array}$ & $\begin{array}{l}A_{I} \\
B\end{array}$ & $\begin{array}{l}4,2 \\
4,6\end{array}$ & $\begin{array}{l}9,7 \\
6,2\end{array}$ & $\begin{array}{l}34, I \\
28,5\end{array}$ & $\begin{array}{l}27,4 \\
35,6\end{array}$ & $\begin{array}{l}19,3 \\
25,1\end{array}$ & $\begin{array}{l}3,1 \\
0,3\end{array}$ & $\begin{array}{l}5,4 \\
0,3\end{array}$ & $\begin{array}{l}\text { nul } \\
\text { nul }\end{array}$ & 19,4 \\
\hline $\begin{array}{l}\text { Sol } \\
\text { podzollque }\end{array}$ & $\begin{array}{l}A_{1} \\
B_{2}^{2}\end{array}$ & $\begin{array}{l}4,2 \\
4,4 \\
4,0\end{array}$ & $\begin{array}{l}36,8 \\
28,4 \\
23,2 \\
\end{array}$ & $\begin{array}{l}27,2 \\
37,8 \\
30,1\end{array}$ & $\begin{array}{l}18,8 \\
27,6 \\
26,2\end{array}$ & $\begin{array}{r}4,1 \\
3,1 \\
16,2\end{array}$ & $\begin{array}{c}5,4 \\
\text { traces } \\
0,6\end{array}$ & $\begin{array}{l}7,4 \\
0,2 \\
0,5\end{array}$ & $\begin{array}{l}\text { nul } \\
\text { nul } \\
\text { nul }\end{array}$ & $\begin{array}{r}6,4 \\
1,9 \\
10,2\end{array}$ \\
\hline $\begin{array}{l}\text { podzol } \\
\text { humo-ferrugine }\end{array}$ & $\begin{array}{c}A_{I} \\
\times A_{2} \\
B_{I} \\
B_{2} \\
. . .\end{array}$ & $\begin{array}{l}3,9 \\
4,5 \\
4,1 \\
4,7\end{array}$ & $\begin{array}{l}38,1 \\
53,2 \\
26,8 \\
47,2\end{array}$ & $\begin{array}{l}36,9 \\
38, ? \\
45,2 \\
41,3\end{array}$ & $\begin{array}{l}7,4 \\
3,2 \\
4,1 \\
2,7\end{array}$ & $\begin{array}{r}2,4 \\
1,2 \\
16, ? \\
2,0\end{array}$ & $\begin{array}{c}6,4 \\
\text { trsces } \\
5,3 \\
3,1\end{array}$ & $\begin{array}{c}12,3 \\
\text { traces } \\
6,8 \\
3,1\end{array}$ & $\begin{array}{l}\text { nul } \\
\text { nul } \\
\text { nul } \\
\text { nul }\end{array}$ & $\begin{array}{r}17,8 \\
0,7 \\
26,2 \\
9,2\end{array}$ \\
\hline $\begin{array}{l}\text { Podzol } \\
\text { huss que }\end{array}$ & $\begin{array}{l}A_{I} \\
A_{2}^{B} \\
(B C)\end{array}$ & $\begin{array}{l}6,9 \\
7,0 \\
6,8 \\
7,2\end{array}$ & $\begin{array}{l}57 \\
57 \\
32 \\
55\end{array}$ & & $\begin{array}{r}12,5 \\
20,2 \\
7,8 \\
28,0\end{array}$ & $\begin{array}{l}I 9,2 \\
15,8 \\
30,4 \\
I 3,5\end{array}$ & $\begin{array}{c}7,6 \\
\text { traces } \\
2 \mathrm{I}, 2 \\
\text { traces }\end{array}$ & $\begin{array}{c}22,8 \\
0,8 \\
27,2 \\
\text { traces }\end{array}$ & $\begin{array}{l}\text { nul } \\
\text { nul } \\
\text { nul } \\
\text { traces }\end{array}$ & $\begin{array}{l}81,2 \\
0,9 \\
2,4 \\
8,1\end{array}$ \\
\hline
\end{tabular}

Tous ces résultats sont exprimés en pourcentage de terre fume séchée â riair.

\section{TABLEAU II}

calcaire de (BC) proviennent de la décomposition des ferro-magnésiens trachytiques. Cette décomposition est très rapide et il semble que les bases soient immédiatement utilisées par les racines qui sont à leur niveau. 


\section{c) l.e sol brun forestier.}

I.e Fageto-Scilletum dans son facies typique est généralement supporté par un sol brun forestier dont nous avons pu observer de nombreux échantillons et profils.

L'analyse se rapporte à un profil du flanc nord du Montchal, audessus du lac Pavin. La roche-mère $y$ est une coulée basaltique d'âge probablement quaternaire, ce qui se traduit par une légère tendance à la mélanisation du sol.

Le profil est du type A B C, avec trois horizons différenciables sur le terrain par une structure assez nettement polyédrique de B. Cet horizon B est cependant un peu lessivé, l'indice d'entrainement des colloïles minéraux est de 1,4. L'analyse minéralogique at binoculaire nous a permis de reconnaitre dans les sables de cet horizon des ferromagnésiens peu attaqués, de la serpentine, de la chlorite et des feldspaths altérés, parfois un peu de damourite. Ia capacité d'échange des bases est relativement élevée en $A_{1}$, ce qui est dù à la nature basaltique de la roche-mére; c'est d'ailleurs un fait dobservation bien connu que la plus grande fertilité des ooulées de basaltes décomposées.

Un profil observé en forêt de Vaucoux, sur roche-mère granitique nous montre un sol très comparable, bien que plus clair et probablement moins riche en bases.

\section{d) Le sol brun lessivé:}

Dans une coupe du sol provenant de Chaudefour, nous avons analysé les horizons. L'horizon B se reconnait très bien sur le terrain par sa couleur nettement rougeâtre. Effectivement, il s'agit d'un sol brun lessivé.

Nous avons noté ine teneur assez élevée en colloïdes minéraux at niveau de l'horizon $\mathrm{B}$ et sturtout l'analyse minéralogique nous a fourni à ce niveau de belles petites boules de limonite. L'enrichissement en fer se traduit donc de manière nette. Ia roche-mère est une cinérite très fine ce qui explique la richesse de tout le profil en éléments fins et la capacité d'échange assez élevée permettant une bonne nutrition de la forèt. Il supporte généralement un FagetoLuzuletum dont les $\mathrm{pH}$ ì $5 \mathrm{~cm}$ de profondeur sont de Fordre de 5.2 .

\section{e) Le sol lessivé (Parabraunerde).}

L.humus devient du type moder et le $\mathrm{pH}$ s'abaisse à 4,7 pour le sol lessivé dont nous avons observé un échantillon dans la hêtraie du Montcineyre. Bien que la roche-mère soit basaltique, I'horizon $\mathrm{A}_{2}$ est très nettement décoloré sur des étendues vastes et il est possible que cela explique la dénomination de la montagne (mons cinerea), les roches affleurantes étant souvent noirâtres au con- 
traire. Dans cette hypothèse, on devrait admettre une assez grande ancienneté de formation de ce sol lessivé, II semble cependant sétre constitué sous la hêtraie en place.

11 existe un horizon $A_{1}$ épais d'une dizaine de centinetres et très foncé. Les feuilles mortes s'y décomposent selon le mode classique. L'analyse ninéralogique montre près de $25 \%$ de limonite dans les sables (grossiers et fins) de l'horizon B. Ce dernier est de couleur marron-chocolat car l'entrainement des colloides humiques est loin d'être négligeable. La richesse en bases de la rochemère permet cependant de conserver une valeur acceptable en bases échangeables.

\section{f) Le sol brun podsolique.}

L'évolution du sol est un peu différente dans le cas des sols bruns podzoliques dont nous avons noté la fréquence sous le FagetoDeschampsietum. Ce type de sol se forme essentiellement dans les cas où lat pente du sol est accusée et le profil analysé vient d'un individu d'association de la hétraie de Sarrevieille à pente d'environ $55^{\circ}$.

On ne rencontre pas dhorizon $A_{-}$net mais un $A_{1}$ très foncé recouvert d'un humus de type moder. Le lessivage très oblique dans les stations à pente aussi prononcée et la finesse de la rochemère cinéritique expliquent la migration des collowiles humiques qui colorent tout le profil. Les collö̈des minéraux sont lessivés obliquement et entrainés en contre-bas, se répandant sur toute lépaisseur du profil des terrains les plus bas. On remarque la rareté des sables supérieurs à $2 \mathrm{~mm}$ causée par la nature de la roche-mère. C'est également sa finesse qui permet un fort pourcentage de colloides minéraux à bases échangeables.

\section{g) l.e sol podsolique.}

le Fageto-Deschampsietum vaccinietosum sétablit sur des sols podzoliques à humus du type mor. Il ne provient que peu de la décomposition des feuilles de hêtres, mais de celle des très nombreuses feuilles de myrtille et de callune. I a couche de fermentation est parfois épaisse d'une dizaine de centimètres. Un exemple de tels sols a été pris dans la hêtraie de Chaudefour. Il existe un hrizon $A_{z}$ décoloré très net et l'horizon $B$ est ocre-rouge. Iit texture du sol est plus sableuse que dans les profils précédents, Lientrainement des colloïdes minéraux est très fort et de l'ordre de 3,9. Minéralogiquement, la limonite est dominante dans l'horizon B alors qu'elle est pratiquement inexistante dans thorizon $\mathrm{A}_{2}$.

\section{h) Le podzol humo-ferrugineux.}

Ce terme ultime de la dégradation des sols est atteint dans les individus d'association les plus acidiphiles du Fageto-Deschampsie- 
LES FORÊTS DU VERSANT SUD DES MONTS DORES

131

I.

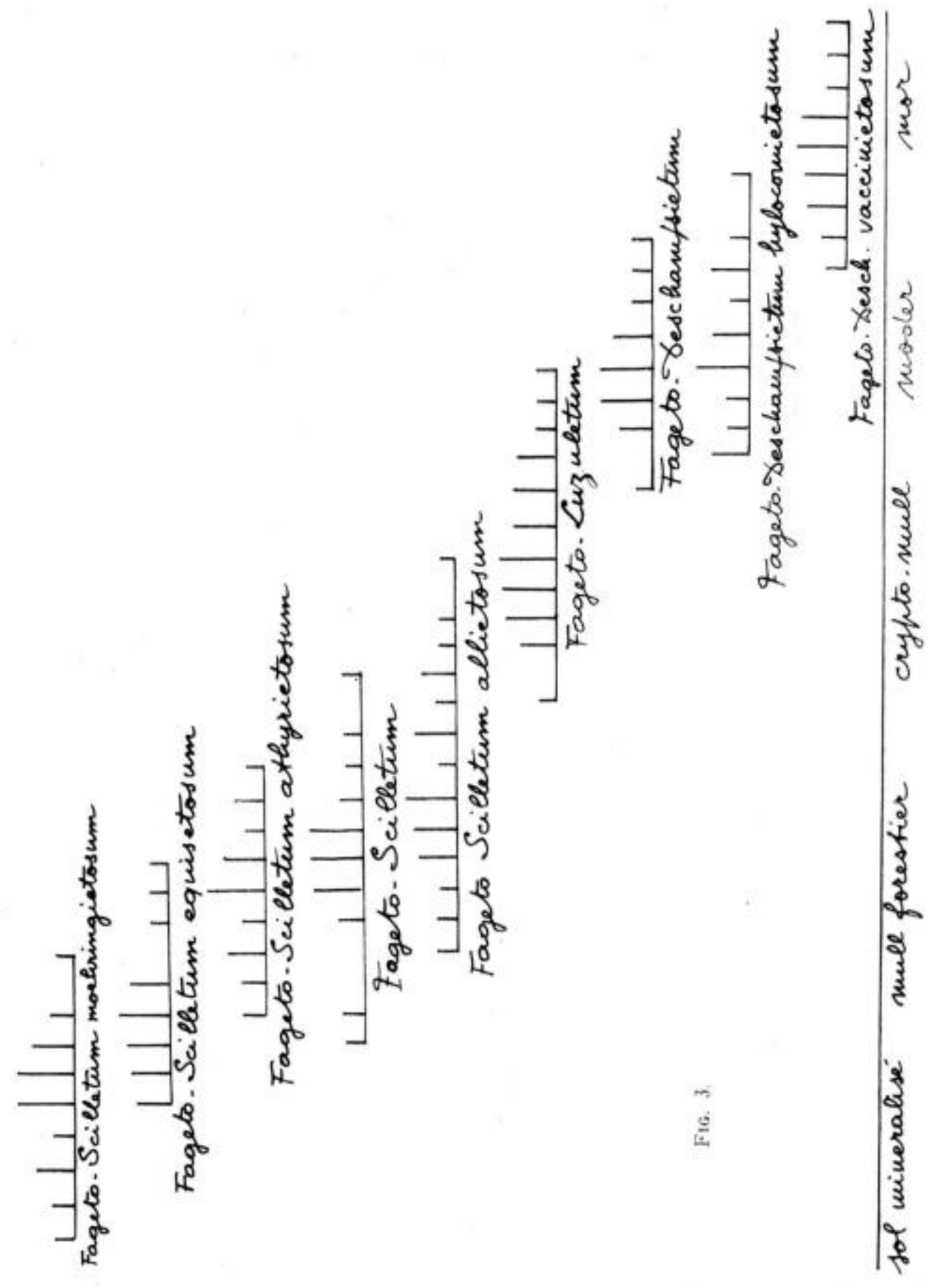


tum vaccinietosum. Sur tun tel sol, la callune est très abondante et le hètre en équilibre vital précaire souvent appelé à disparaitre.

L.horizon $\mathrm{A}_{2}$ est constitué presque uniquement de sables, alors que $B_{1}$ est beattcoup plus riche en minéraux. I. indice d'entratise-

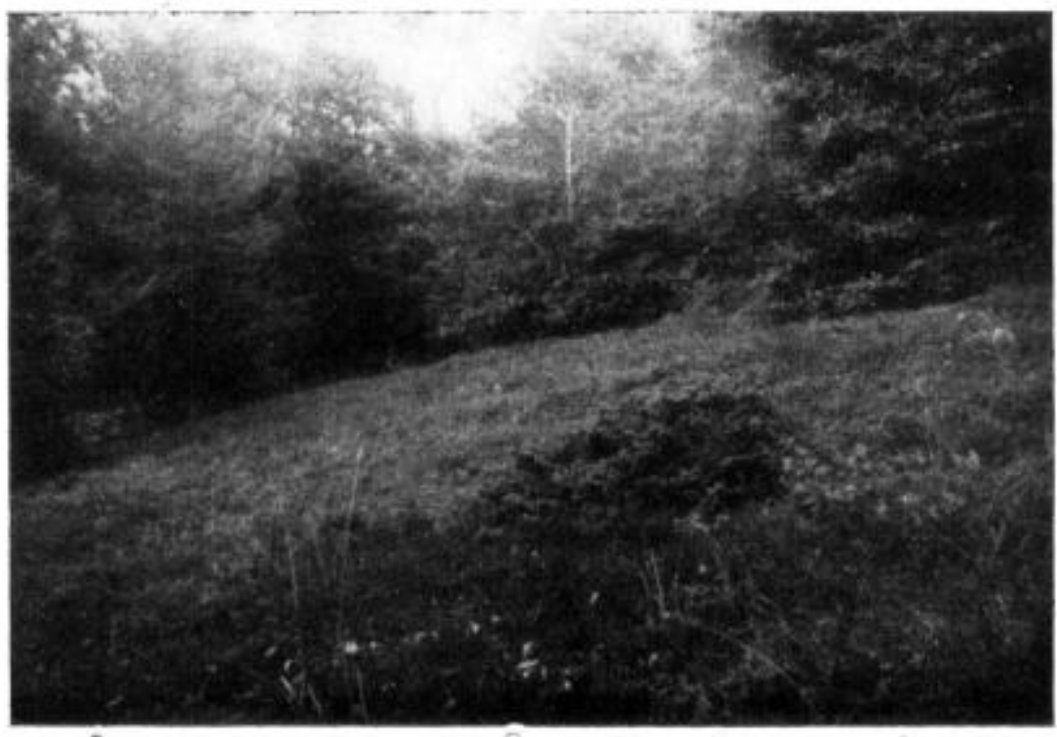

Clairière sur sol très acidifié

Bruyère, callune et genévrier commun s'emparent du sol, Ia reconstitution de la foret y semble très difficile.

(C1. Cusst.)

ment est proche de $8 . \mathrm{B}_{2}$ est surtout ferrugineux et la limonite en constitue l'essentiel. La capacité d'échange est presque nulle clans l'horizon $A_{2}$ mais se releve dans $B_{1}$ ou les racines puisent lessentiel de leur nourriture.

i) Le sol hydromorphe du bois du Pavin.

Dans une hêtraie près du lac Pavin, assez dégradée par les nombreux touristes, nous avons rencontré un type de sol différent. Les horizons $A_{1}$ et $A_{2}$ sont proches de ceux d'un sol brun lessivé comme il en est au voisinage, mais les horizons B semblent appartenir à un podzol hydromorphe. L'humidité est très grande, des sources s'écoutlant vers le lac entretenant une nappe phréatique permanente à une quarantaine de centimètres de profondeur. Nous rapprochons ce sol des types décrits par Duchaufour dans les Landes".

* Ces sols ne sont pas identiques mais le facteur principal de leur formation - les remontées d'eau à partir d'une nappe importante voisine - est le même. 
L'horizon $\mathrm{B}_{2}$ est faible et n'apparait guère que comme un liseré rouge discontinu. Par contre, $\mathrm{B}_{1}$ est épais d'une vingtaine de centimètres, très noir et à consistance dure: c'est un véritable alios humique. Nous considérerons done ce sol comme un podzol humique a alios. Il semble rare dans notre dition, les montagnes en pente accentuée n'en permettant qu'exceptionnellement la genèse.

Nous n'avons pas d'analyse du sol de Fageto-Scilletum equisetosum mais un profil nous a montré la présence d'un horizon bleuàtre. II s'agit done d'un sol à pseudogley comme nous le supposions en 1960. I a présence permanente d'une nappe d'eau l'explique aisément.

L'acidité iomique du sol a été résumée dans la figure 3 montrant lamplitude du $\mathrm{pH}$ prour chaque association. Nous le discuterons plus loin.

\section{3 - Les facteurs biotiques.}

La compétition biotique entre les espéces se traduit de trois facons: par la périodicité de la végétation, par la concurrence vitale des espèces en présence et par les formes biologiques dominantes.

Depuis I. focong. la périodicité de la flore des hétraies a été décrite assez souvent. ( ) n peut distinguer:

- un groupe conservant ses feuilles en hiver. mème sous la neige: dans nos forèts, il comprend notamment les myrtilles et les callunes dans la strate arbustive (encore qu'elles perdent un bon nombre de leurs feuilles), et dans la strate herbacée Lusula maxima. Lusula pilosa, l.usula nivea, Oxalis acetosella. Asperula odorata. Veronica officinalis, Deschampsia flexuosa, Bromus asper, Festuca heterophylla qui conserve ses feuilles basales, de méme que les Carex, Les fougères restent marcescentes (Athyrium filix-foemina, Dryopteris filix-mas, Aspidium aculeatum, Polystichum spimulosum...). Toutes les muscinées continuent également à assimiler pendant la saison hivernale.

- un groupe ayant en hiver, entre les restes des feuilles desséchées de jeunes pousses sorties en automne: Milium cffusum, Poa nemoralis, Melandryum silzestre, Ramunculus brevnimus. Viola silz'estris, Epilobium montanum et durieni, Myosotis sikatica, Galcobdolon luteum, et tous les Hieracium à stolons.

- un groupe " prévernal n (SALISBURY) héliophiles apparaissant at printemps et disparaissant dès que lombre des arbres devient épaisse: Anemonc nemorosa, Scilla lilio-hyacinthus. Corydalis solida. Adora moschatellina, Conopodium denidatum. Pulmonaria affinis présente une phase de vie intense (avec floraison) au printemps et les feuilles radicales d'été sont bien différentes. 
- un groupe plus sciaphile stacommodant de l'ombre des arbres: Stellaria nemorum, Orobus tuberosus, Maianthemum hifolium, Presanthes purpurea, Solidago sirga-aurea, Phyteuna spicatwm, Cicerbita muralis, Pirola sp.

Notons que les hètraies des Quercetalia présentent une végétation assez uniforme toute l'année, les végétaux dominants appartenant au premier groupe, alors que les hêtraies à scilles montrent deux phases bien distinctes. Lemík avait déjà signalé dans le Perche que "les associations du Quercion se distinguent par la présence d'espèces toujours vertes et l'ahondance d'espèces à assimilation continue n. 11 semble que la continuité de l'assimilation chlorophyllienne puisce compenser dans ces associations la pauvreté du sol.

Il peut ètre intéressant de rapprosher les espèces de chaque alliance, Fagion ou Quercion, pour examiner leur adaptation à chaque sol: Sont en concurrence les espèces à aptitudes écologiques voisines. Les espèces du Fagion sont généralement liées au mull. Srilla lilio-hyacinthus. Stellaria nemorum ssp. glochidisperma, Pulmonaria affinis ssp. etu-affinis. Conopodium denudatum. Galeohdolon luteum, Myosotis silvatica, Festuca silvatica, Impatiens noli-tangere, Milium effusum, et Neottia nidus-aris sont exclusives du mull dans notre dition thut at moins. Phytewma spicatum ssp. oclurolewcum. Asperula odorata, Denteria pinuata. Cicerbita muralis, et Poa nemoralis s'accommodent également d'humus de type moder.

Les espèces du Quercion sont lices an contraire aux humus acides, Elles n'apparaissent guère au-dessus de pH 6. Hieracium murovum sensu lato, Sorhus aria, Pirola minor. Consallaria mainlis. Teucrium scorodonin sont dans ces cas. Solidagn airga-aurea et Deschampsia flexwosn montrent une amplitude un neu plus grande.

Les espèces du Querceto-Fagetea qui doivent s'adapter à toute la gamme des conditions écologiques précédentes ont effectivement la possibilité de végéter anssi bien sur le mull que sur le mor. Viola silvestris. Ancmone nemorosa. Melandryum silvestre. Rromus asper. Ranunculus brevnimus se rencontrent de $\mathrm{pH} 7$ à $\mathrm{pH} 4$.

Les différentielles de chaque assnciation ou sous-association sont évidemment liées de manière assez stricte aux conditions écologiques les plus fréquentes dans ce groupement. Geranium robcrtianum. Equisefum hvemale, Vaccinium myrtillus et Calluna aulaaris nous semblent exclusives de leurs groupements, à l'intérieur de la hêtraie. Leucobryum glaucum est lié an mor.

Ta concurrence vitale peut également se reconnaitre à la prééminence de telle ou telle forme biologique. On sait l'importance que cette notion revêt pour la comparaison de grounements à flores đifférentes dans des pays éloignés.

Les spectres biologiques, étahlis d'après la classification de RaunKIAER, se révèlent assez semblables pour tous nos groupements. Les 
hémi-cryptophytes dominent de façon générale; les géophytes sont nombreux; les phanérophytes lien représentés. Les thérophytes penvent etre absents et ils sont toujours rares. Nous avons établi les spectres biologiques en tenant compte des coefficients d'abondancedominance dans chaque groupement (fig. 4). Les abréviations représentent respectivenent: F.S. - Fageto-Scilletum typique: F.S. Ath. = Fageto-Scilletum athyrietostm ; F.S. Eq. = Fageto-Scille-

\section{SPECTRES BIOLOGIQUES}
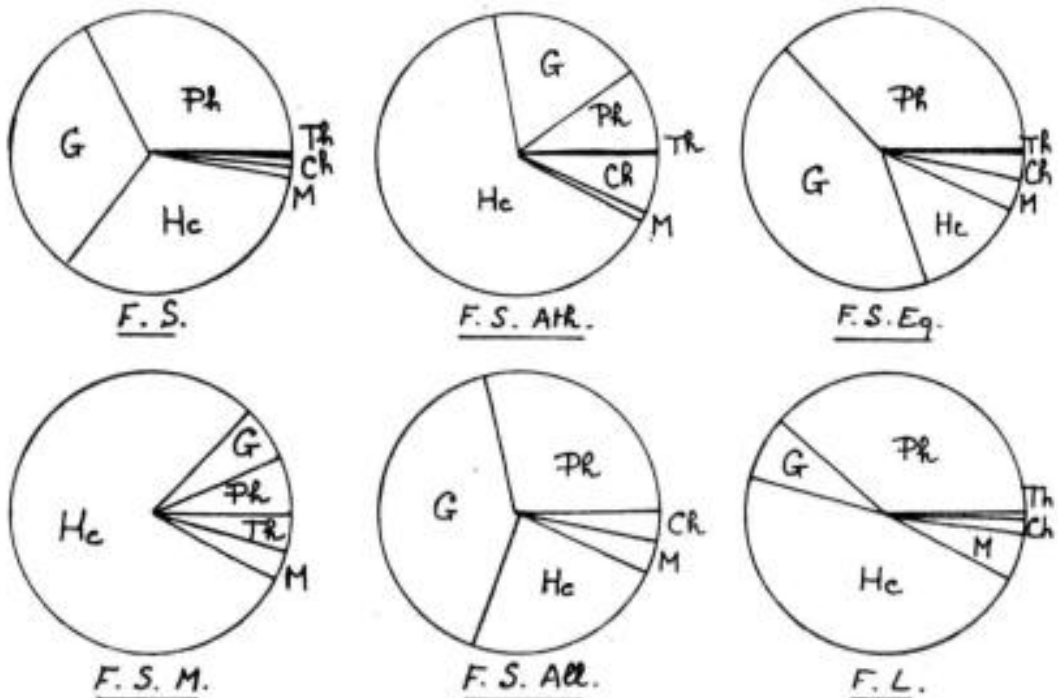

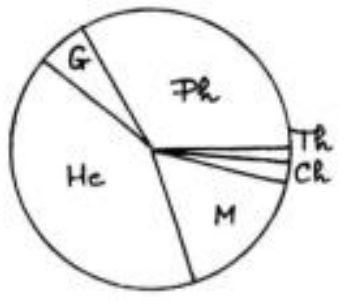

F.D.

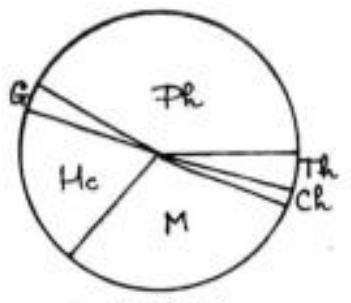

F.D.H.

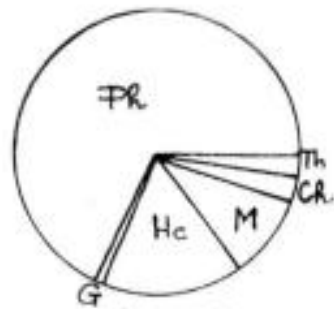

F.D.V.

FIG. 4.

tum equisetosum; F.S.M. = Fageto-Scilletum moehringietosum; F.S.All, - Fageto-Scilletum allietosum; F.I. = Fageto-Luzuletum:F.D. - Fageto-Deschampsietum: F.D. = Fageto-Deschampsteium; F.D.H. - Fageto-Deschampsietum hylocomietosum ; F.D.V. = Fageto-Deschampsietum vaccinietostum. 
Ce mode de représentation reflète beaucoup mieux les écarts entre nos divers groupements. De manière générale, les géophytes se font de plus en plus rares au fur et à mesure de l'acidification du sol. Bien que celui-ci soit assez profond pour eux, ils ne trouvent plus une nourriture suffisante au niveau de l'horizon $\mathrm{A}_{2}$ appauvri. Au contraire, les phanérophytes, et surtout les nanophanérophytes qui exigent un support moins stable, végètent facilement en atteignant l'horizon d'accumulation. Les muscinées profitent de la pellicule superficielle oit leurs rhizoïdes sont ancrés pour végéter un peu sur tous les milieux: néanmoins elles sont plus rares sur les mors trop acides (excepté Leucobryum flaucum). Sur le mull elles sont étouffées par les especes herbacées plus vigoureuses.

Dans le Fageto-Scilletum athyrietosum les hémi-cryptophytes ont une grande importance: poussant leurs rhizomes ou leurs racines dans les blocs de rochers et dans le sol peu profond, ils consolident le sol et l'approfondissent. Ce sont eux qui jouent le róle dynamique le plus important dans la formation du sol forestier. Les géophytes n'ont pas assez de place pour implanter des bulbes ou des organes souterrains importants. Dans le Fageto-Scilletum moehringietosum où nous avons noté un éclairement relatif de $25 \%$. les thérophytes prennent une place notable. C'est celle de nos associations dont le pourcentage d'espèces annuelles est nettement le plus fort. Att contraire. les thérophytes disparaissent complètement dans le Fageto-Scilletum allietosum dont l'altitude est trop élevée. Te recouvrement du sol une lonne partie de l'année par la neige et les températures extrêmes minimales certainement très basses n'autorisent pas l'implantation de thérophytes fragiles. Par contre les géophytes bien protégés par la neige sont favorisés. Du Fageto-I uzuletum au Fageto-Deschampsietum hylocomictosum on voit l'accroissement du pourcentage des mousses, aux dépens des hémicryptophytes dont les racines superficielles sont en concurrence étroite avec les rhizoides et dont les racines profondes sont dans un horizon appauvri. Dans le Fageto-Deschampsietum vaccinietosum, les phanérophytes deviennent très abondants. L'éclairement réduit sons leur feuillage et la podzolisation du sol réduisent le nombre dautres végétaux.

\section{D - Le dynamisme des hêtraies et des associations non silvatiques du même étage}

Dans cette partie de notre étude nous ferons appel à un certain nombre de groupements définis par DE LAciafeil.te en 1961. Nous avons d'ailleurs confronté souvent nos vues et nous lui sommes redevable de précisions très intéressantes*. Nous donnerons, pour rand.

* Voir son mémoire déposé au Laboratoire de Botanique de Clermont-Fer- 
faciliter la compréhension, les caractéristiques sommaires de ces associations.

\section{1 - Le passé de la végétation.}

On connait des vestiges ante-dinantiens de la vie végétale en Auvergne, mais cest seulement à partir du Tertiaire que nous avons affaire à des plantes semblables à celles que nous voyons actuellement. Grangeon, dans sa contribution à l'étude de la flore stampienne de Limagne a groupé les " plantes des forêts sociales et montagneuses ". Nous relevons dans la liste qu'il donne: Thuiopsis curopaca, Abies sp., Podocarpus gypsorum, Protophyllocladus, Fagus gautieri. Cependant l'état de conservation des empreintes nous semble ne pas permettre d'être très affirmatif sur l'attribution de ces espèces à un genre déterminé; c'est ainsi que, comme le signale Grangeon, l'empreinte dénommée Protophyllocladus avait déjà été rangée parmi les Fougères, les Cycadées et les Taxacées. La présence de Podocarpus sp. nous semble poser un intéressant problème de phytogéographie; ce genre a été également reconnu dans le Tertiaire d'Huppaye (Belgique), de Cervera (Catalogne), d'Aix-enProvence. d'Häring (Tyrol), de Koumi (Ile Eubée) et Leoben en Styrie. On sait qu'il s'agit actuellement d'une espèce strictement sud-équatoriale. S'agit-il bien d'un vrai Podocarpus?

Fagus gauticri Laurent et Marty est représenté par plusieurs empreintes. Il s'agit " de petites feuilles d'aspect coriace, de 25 $35 \mathrm{~mm}$ de long sur 9-12 de large munies de 10-12 paires de nervures secondaires $»$. Il serait "apparenté au groupe silzatico-orientalis n. Dans les cinérites du lac Chambon, on rencontre des feuilles de hêtre d'attribution indubitable. Rapproché de Faqus moesiaca, ou même dénommé Fagus silvatica par MARTy, cet arbre aurait constitué des forêts au Pliocène (au Villafranchien pour RuDEL).

Les cinérites du Cantal nous ont conservé l'image de l'étagement botanique du Tertiaire terminal. Les forêts s'étendaient à partir d'un étage à Abies ramesi. On y rencontrait Aluus qlutinosa, Populus tremula et Cormus sanquinea, mais aussi Sassafras ferretianum, Acer loetum, Jasminum heterophyllum et Berhemia volubilis. Dans la strate herbacée, les fougères sont les mieux conservées, Dryopteris filix-mas, Polypoditm vulgare. On reconnait aussi Hex aquifolium, Hedera helix et Vinca minor, espèces à feuilles coriaces se fossilisant bien.

Dès le Villafranchien, l'élément euro-sibérien parait le mieux représenté sur le Plateau Central comme l'a souligné Braun-Bu.aNQUET en 1921. Il a gardé cette prééminence jusqu'à nos jours. CHAsSAGNE écrit en 1956: "Les types sibériens sont fréquents et répandus en Auvergne; le plus grand nombre est arrivé assez récemment et cette marche se poursuit à l'heure actuelle n. 
L'évolution de la végétation au Quaternaire est maintenant bien connure dans notre région grâce anx travaux de G, et C. Dunoss et de L.mós. I. analyse pollinique a été appliquée à de nombreuses stations. Après le maximum du Würm, la forêt semble absente des sommets et les pentes devaient ètre recouvertes de bouleaux, probablement de Betula nana, que lon trouve actuellement dans le nord de la Margeride, en Haute-I.oire entre Grèzes et Chalaneilles et en Lozère vers I ajo. Un réchauffenent temporaire pernet enstiite la remontée de la forêt vers $1200 \mathrm{~m}$, avec un mélange de botrleaux et de pins. Les bouleaux semblent appartenir aux espèces zrrrucosa et pubscens. Après un refroidissement amenant une toundra temporaire, la forêt réapparait en mélange de bouleattx, de pins et de noisetiers. Puis les pins diminuent an profit du noisetier et surtout du chène. T'orme s'étend, le hêtre. le sapin et l'aulne commencent à étre présents. Le chène semble avoir atteint $1400-1500 \mathrm{~m}$. ce qui est presque la limite supérieure actuelle du hêtre. Le tillenl prend ensuite une importance notable. Ia phase suivante voit un maximum du sapin et du hêtre. "La limite supérieure était entre 1400 et $1500 \mathrm{~m}$ dans les endroits abrités, s'alaissant à moins de $1400 \mathrm{~m}$ dans les endroits exposés aux vents. conme la limite naturelle actuelle n (L.FMÉE - 1942). Fnfin, le sapin se raréfie et disparait totalement à l'état spontané du versant sud des Dores et le hêtre s'étend considérablement. C'est la phase actuelle qui est marquée par la totale subjugation de la nature par l'homme. II est hors de doute que le xvmio siècle a été une période de débrisement très important en Auvergne. L.vourt cite Juvénal Hr.ruEt "qui constate la destruction presque générale des forêts de la province $v$. Fn 1828. les procès-verbaux ne signalent que "quelques rares futaies, assez mal venues w. Depuis 1850 , la diminution de la densité de la population montagnarde. la disparition des troupeaux de chèvres ef. plus récemment, l'action de l'Administration forestière ont permis la reconstitution d'une honne partie de la forèt primitive.

\section{2 - Dynamisme des associations.}

Caractérisons d'abord rapidement les associations non-silvatiques dont nous allons parler.

L.e Centaureo-Cynosuretum Br. et Tüxen 1949 est représenté par de belles prairies entre 1050 et $1200 \mathrm{~m}$, pacagées ou clóturées pour la fauche. Ils se situent sur des pentes ne dépassant pas $10^{\circ}$. L'association se reconnait à Cynosurus cristatus, Trifolium repens. Centaurea nigra. Phletm pratense et Senecio jacobea.

L'assèchement du sol ou un pacage abusif transforment cette association en Nardeto-Leontodonetum pyrenaici (DE. I.ACHAPELLE). Cette dernière se caractérise par la grande abondance du nard, Leontodon pyrenaicus, Meum athamanticum, Selinum pyrenaeum et Ga- 
lium hercynium. On y rencontre de nombreuses transgressives de lat hêtraie, spécialement dans la sous-association à Chrysanthemum delarbrei et Gentiana lutea.

Les falaises trachytiques humides sont occupées par ce que LUQUET nommait " association à Adenostyles albifrons, sous-association à Luzula desvaurii. DE LAchapelle l'a dénommée "I.uzuletum desvauxii $n$, conformément à la nomenclature. C"est un groupement de l'Adenostylion indépendant des autres associations de cette alliance.

\section{a) Hêtraie et associations non silvatiques.}

Dans les zones les plus humides du Nardeto-Leontodonetum, il se forme, par refus du bétail et absence de pacage, une sorte d'aulnaie nitrophile qui renferme de nombreuses especes de la hetraie. Voici un relevé de cette formation:

Alnas glutinosa Gaertu. 1-2; Salix atro-inerea Brot. 1-1; Sali, repens L. + ; Salix aurita L. 1-2; $\times$ Salix ambigua Erhr. +; Betula pubescens Ehrh. + ; Caltha palustris L. 1-3; Ribes petraeum Wulf, + ; Sorbus aria Crtz, + ; Sorbus aucuparia L. +; Impatiens noli-langere L. + ; Crepis paludosa Moench +; Luzula campestris $\mathrm{L}$. + ; Cirsium palustre Scop. +; Calamagrostis arundinacea Roth. +; Orchis latifolia L. + ; Carex div. + ; et les transgressives de la hêtraie: Pulmonaria affinis Jord. 1-2; Stellaria nemorum ssp. glochidisperma Murb. 2-1; Euphorbia hyberna L. + ; Epilobium montanum L. + : P'renanthes purpurea L. + ; Circaea intermedia Fhrh. +; Myosotis silvatica Hoffm, +; Poa sudetica Haenck. +; Melandryum silvestre Roehl. +; Festuca silvatica Will. + ; et Fagus silvatica (Semis) +.

Ce grotupement parait devoir évoluer vers une hétraie à Scilla Liliohyacinthus. On peut noter les points de ressemblance qu'il présente avec le Fageto-Scilletum noehringietosum. Son recouvrement est en effet faible et les conditions écologiques assez semblables.

Le Nardeto-Leontodonetum chrysanthenosum renferme lui aussi, comme l'a justement noté DE LACHAPELLE, de nombreuses espèces de la hetraie. Il ne semble pas ètre cependant un simple groupement de transition entre la prairie et la forêt. I.es principales transgressives de la hetraie sont: Euphorbia hyberna, Solidago virga aurea, Poa sudetica, Hypericum pulchrum, Veronica officinalis, Galeobdolon luteum, Knautia silatica, Teucrim scorodonia, Pulmonaria affinis, Deschampsia flexuosa et Anemone nemorosa. Les individus d'association sont très homogènes, qu'ils soient près de la lisière ou loin d'elle. Le sol est assez évolué, " proche d'un sol brun uniforme $n$ (DE LACHAPELLE). L'observation de germinations de hêtres semble permettre de penser à une colonisation possible (ou à une reconquête) par la forêt si le pacage cessait. 
Le Luzuletum desvauxii occupe les rebords des falaises trachytiques humides. Ceest à leur base, sur un mince ranker à mull que se constitue le Fageto-Scilletum athyrietosum. Si l'érosion des eaux courantes n'entrainait le sol du Luzuletum desvauxii dès qu'il se forme, les fougères ne tarcleraient pas à monter à l'assaut des falaises et à étouffer les luzules sous leur ombre, préparant la marche de la forêt. Ce ranker à mull où s'installe la hétraie évolue vers un sol brun uniforme, stade le plus jeune des vrais sols silkatiques. Si le sol est trop humide par la présence d'une nappe d'eau permanente, il se forme un Fageto-Scilletum equisetosum près de la limite inférieure des forêts; an contraire les conditions écologiques plus rudes aux altitudes élevées conduisent à la formation du Fageto-Scilletum allietosum. Cette sous-association ne se forme pas à l'exposition ouest où le sol est trop lessivé. I.e profil transversal de la vallée de Chaudefour permet de suivre une telle évolution.

Un léger lessivage du sol transforme le Fageto-Seilletum en Fageto-Luzuletum, association pivot des hètraies, En effet, l'éclaircissement naturel de la végétation amène une forte minéralisation de la surface du sol et il s'établit un Fageto-Scilletum moehringietosum, qui permet la reconstitution de la hêtraie typique. Mais le Fageto-I uzuletum peut évoltuer en sens inverse vers une acidification plus potssée de son sol. On arrive alors au Fageto-Deschampsietum, c'est-à-dire que l'on passe dans l'ordre des Quercetalia. Comme Pochon et DF BARJAC l'ont sonligné, un senil biologique important existe à pH 5. Dans nos hêtraies, ce setuil se traduit par une irréversibilité evolutive: en dessous de pH 5, le sol s'acidifie continuellement et la hêtraie tend à se détruire elle-mème*, alor's que le Fageto-Scilletum typique se reconstitue lorsque le $\mathrm{pH}$ n'est pas descendu à 5. C'est également le point où les mycorrhizes commencent à jouer un róle important, par leur noubre propre et par le nombre des espèces atteintes. Elles ont cependant, comme l'ont noté certains auteurs, besoin d'un seuil d'éclairement relatif de lordre de $6 \%$; c'est ainsi que la luminosité relative ne dépassant pas $3 \%$ dans le Fageto-Deschampsietum hylocomietosum les plantes y sont tres rares, à l'exception des muscinés moins exigeantes. Cette sous-association constitue un ensemble par sélection des espèces les plus acidiphiles. En été, la circulation d'eau ascendante se fait essentiellement par les racines des hêtres et le fer ne remonte que difficilement dans le sol, il ne se forme qu'un sol très lessivé a tendances podzoliques, sans horizon ferrugineux net. Au contraire. la grande luminosité de la sous-association à Vaccinimm $(9 \%)$ se traduit par l'abondance des champignons mycorrhiziens et les espè-

* Ce cas ne semble pas totalement identique à celui des hêtraies-sapinières ot̀ ce seuil parait ètre plus bas. Rappelons qu'il s'agit ici de hêtraies pures. Les micro-organismes - et notamment les bactéries - seraient-ils différents? Une éventuelle action des corps contenus dans les aiguilles de sapins serail intéressante à étudier. 
ces à associés fongiques y sont nombreuses. Ils permettent l'utilisation des humus de type nor. L'aboutissement du Fageto-Deschampsietum vaccinietosum est la constitution d'un podzol humo-ferrugineux profond qui convient très bien à la Callune venue des landes voisines. Elle exerce, comme l'ont montré les forestiers anglais, une action inhibitrice nette sur le développement des basidionycètes, et par là mẻne entraine la disparition des mycorrhizes, tout au moins du groupe associé aux espèces herhacées, groupe "différent de celui des espéces ligneuses a (Boullak1) in litt.). La forèt a disparu, victime d'une évolution irréversible dont le départ se situe aux environs de $\mathrm{pH} 5$.

Ces interactions entre le sol et la végétation jouent le róle prédominant dans le dynamisme de la végétation. Il ne faudrait cependant pas oublier l'action des facteurs externes sur l'évolution des sols et donc de la végétation. Ies, pluies dominantes venant de l'ouest, les pentes est et nord-est en sont protégées. Si ces pluies agissent sur le sol, elles accentuent la vitesse de lessivage du sol et done la podzolisation. ("est effectivement sur les pentes outest et sud-ouest que nous avons reconnu la plus grande fréquence du Fageto-l)eschampsietum et de ses sous-associations acidiphiles (fig. 5).

\section{b) La limite suférieure de la hếtraie.}

Il est classique de faire état des défrichements effectués an Xvur siecle pour affirmer que la linite supérieure de la hètraie a été notablement abaissée et que sa véritable altitude devrait être supérieure d'une centaine de mètres à ce qu'elle est actuellement. Nous avons déjà évoqué les résaltats de l'analyse pol'inique (ceux de LEMÉE notamment) permettant d'affirmer que cette limite était au début du néolithique, ćest-à-dire à la phase du sapin et du hétre, sensiblenent la même quactuellement. S'il y a eu une nette action de l'homme sur l'abaissement de la forêt, il faut done supposer qu'elle est mésolithique et il nous semble que la forèt aurait eu le temps de se reconstituer. Les données toponymiques coincident d'ailleurs avec cette maniere de voir, aucun lieu-dit de nom dérivé de "Fagus o est à noter au-dessus de 1400 m dans notre région. Ils sont par contre nombreux (la Faye, Fayet...) plus bas.

Comment se présente donc actuellement la lisière supérieure de la hêtraie? Remarquons tout d'ahord que les versants exposés de façon trop directe aux vents dominants de sud-ouest portent des associations de prairie alpine plus bas que les autres. Nous pouvons estimer la limite forestière supérieure à $1450 \mathrm{~m}$ par exposition abritée et à $1350 \mathrm{~m}$ seulement aux expositions sud et ouest... Selon que la forêt appartient à l'alliance du Fagion ou à celle cu Quercion, il semble que les choses soient légèrement différentes.

Si la forêt est formée sur un sol peu acide. on observe à sa limite supérieure un groupement à Sorbus et Salix, d'une trentaine 
$\Downarrow$
$w$

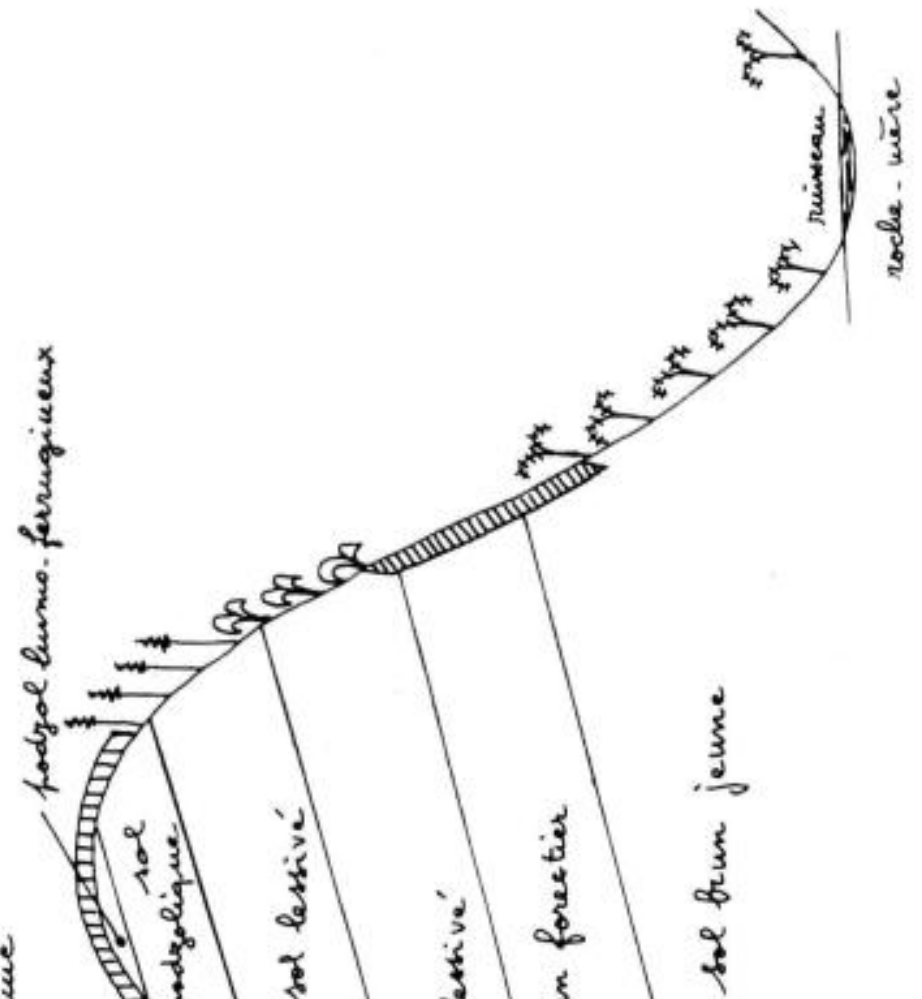

s:

ఏँ

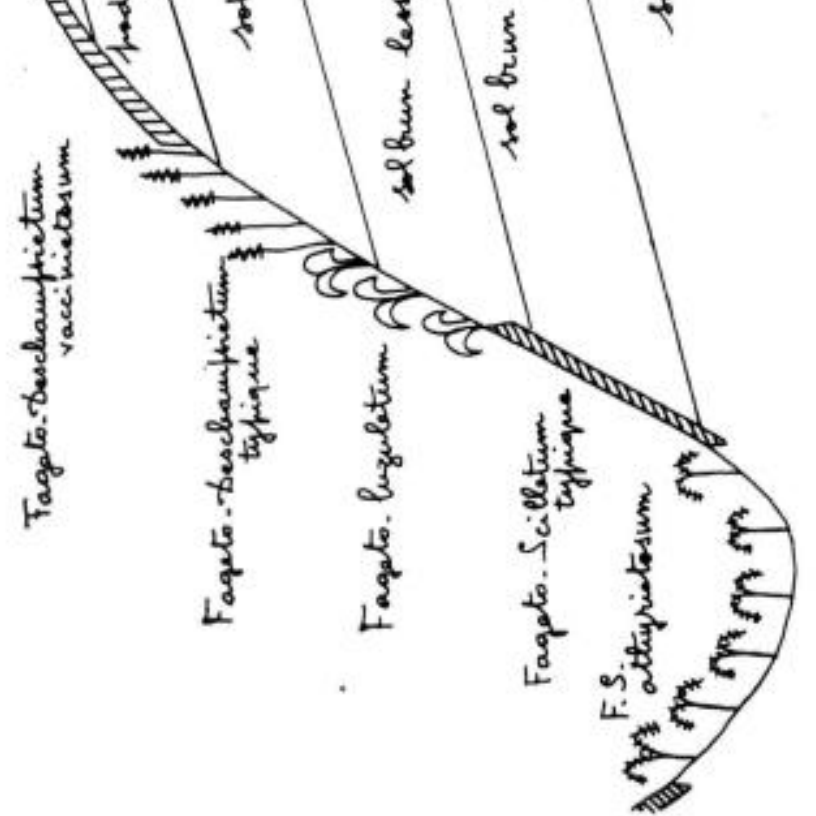


de mètres de large. Ce groupenent étudié par dE LACHAPELLE parait assez homogene. On y note ordinairement des transgressives de la hetraie, Euphorbia hyberna, Melandryum rubrum, Solidago virya-aurea, Senccio cacaisaster. Anemone nemorosa. Allium rictorialis, Lusula maxima, Prenanthes purpurea, et des transgressives de l'Adenostylion. Adenostyles alba, Ranunculus aconitifolius, Aconitum napellus, Lusula desvauxii. Cicerbita phmieri. II existe en plus quelques caractéristiques: Aconitum lycoctontm, Geranium silvaticum, Trollits europeus notamment.

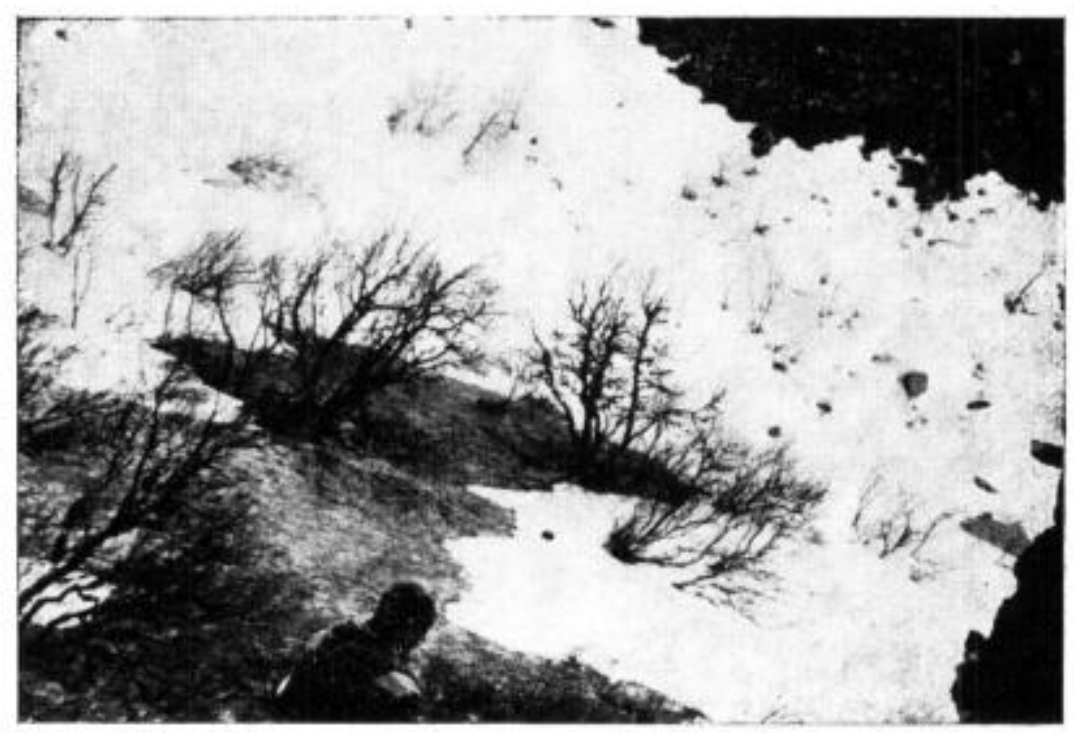

Limite superieure atteinte par la hétraic $(1545 \mathrm{~m})$.

1.a photo, prise au mois de mai, montre un des névés de Chaudefour.

Les hetres sont rabougris et couchés jar les avalanches.

(C1. Cusset.)

Au-dessus des hétraies de l'ordre des Quercetalia, on ne retrouve tien de tel. Il existe un groupement à Jumiperus, distinct des landes subalpines. On y relève: Juniperus communis, Calluna vulgaris, Vacinimm myrtillus, Deschampsia fleruosa, Campanula linifolia, Potentilla erecta. Betonica officinalis, Orobus tuberosus, Dianthus silvaticus, et Cytisus purgans.

11 faut enfin faire une place particuliere au Nardeto-Festucetum rubrae (DE Lachapelle). BraUn-Blanouet parlait à propos d'un groupement comparable de "groupement semi-naturel créé par le pacage occupant des sols de vocation forestière ". Nous n'avons pas observé de troupeaux à une altitude aussi élevée mais estimons qu'il s'agit bien là d'un ensemble que la hêtraie pourrait facilement 
coloniser. On y rencontre surtout Nardus stricta, Viola sudetica, Festuca rubra, Alchemilla vulgaris, Anthoxantum odoratum, Phlewm alpimu, Lusula sudetica. Trifolium alpinum. Selinum pyreneum. Meum athamanticum, Potentilla aurea, Conopodium denudatum, Anemone nemorosa, L.ilium martagon, Veronica officinalis, Vaccinium myrtillus, Gentiana lutea, Dianthus silvaticus. Ajuga repens. Enphrasia nemorosa, Jasione perennis, Cerastium roespitosum. Rumer acetosella, Ramunculus nemorosus. ...

\section{c) Les associations de coupc.}

Là encore on assiste à un dualisme entre les hètraies des Fagetalia et des Quercetalia.

Pour les forèts à sol peu acide. on voit dès la première année l'apparition d'une série d'especes nitratophiles, qui profitent de l'action minéralisante des bactéries de surface. Il apparait alors une association à Epilobium spicatum, un Epilobietum spicatae. On observe d'abord Epilobium spicatum nettement dominant. Galeopsis tetrahit, Digitalis purpurea, Urtica dioica. Senecio fuchsii, Galeohdolon lutetr. Lapsana communis, Geranium phoeum, Geranium robertianum. Glechoma hederacea, Impatiens noli-tanyere. Funaria hygrometrica, Ceratodon purpureus, et Polytrichum formosum. Puis les phanérophytes rudéraux s'installent, Sambucus racemosa et Rubus idacus. Une dizaine d'années après la coupe, on rencontre: Sambucus racemosa, Rubus idacus, Urica dioica, Salix sp. Corylus azellana, Cicerbita muralis, Deschampsia flexuosa, Pulmonaria affinis, Oxalis acetosella, Phyteuma spicatum. Solidago virgaaurea, Ribes petracum. Glechoma hederacea, Senecio cacaliaster. Pteridiun aquilinum, et mème quelques semis de Fagus. I.a hètraie ne va pas tarder à se reconstituer, hêtraie du type Fageto-Scilletum.

Pour ce qui concerne les hètraies des Quercetalia, la régénération est beaucoup plus difficile et parfois méme impossible. Voici un relevé où la repousse de la hêtraie parait possible: Sarothamuns scoparius 2-1: Rubus sp. 1-1: Vaccinium myrtillus 1-2: Deschampsia flexuosa 2-1: Pteridium aquilinum + ; Betonica officinalis + ; Epilobium spicatum +: Agrostis vulgaris +: Veronica officiualis + . Au contraire, lorsque la callune s'installe après une coupe, it semble bien que le sol ait perdu toute vocation forestière*. Une clairière dans un lambeau de hêtraie près de Courbanges ne nous a pas montré le moindre signe de reconstitution de la forêt depuis quatre ans que nous avons observé son évolution. On y trouve: Calluna vulgaris 4-2; Juniperus communis 1-2; Vaccinium mvrtitlus 1-3; Betonica officinalis + ; Orobus tuberosus + ; Deschamp-

* Il s'agit ici de lévolution nafurelle de notre dition. Des reboisements seraient prohablement possibles, mais délicats. Rappelons que f'épicéa n'est pas spontané dans. les Dores. 
sia flexwosa + ; Campanula rotundifolia + ; Pirola minor + : Potentilla crecta $+;$ Hylocomium splendens $1-3 ;$ Hypnum schecberi + : Leucobryum glaucum + : Hypnum cupressiforme ssp. + .

\section{E - Les forêts de l'étage inférieur à la hêtraie}

Il est plus difficile de décrire le passage des hètraies aux forèts d'altitude moindre, que la limite supérieure de la hêtraie. Nous tenterons d'en donner une esquisse après avoir examiné la composition de ces forêts. Nous en prendrons des exemples dans la série forestière que lon rencontre de Roche-Charles à Courgotil le long du ruisseau de Sauit. Il est regrettable qu'il n'existe atcun chemin dans cette région fort instructive. Ixes altitudes vont de $1040 \mathrm{~m}$ près de Boslabert à environ $600 \mathrm{~m}$ vers Lagouzoux.

L.es bois sont de nature assez complexe et montrent sotvent un véritable enchevêtrement d'associations différentes, selon des conditions écologiques elles-mêmes très variables. II ne nous a pas été possible d'étudier de façon précise ces dernières et nous le regrettons. On peut dégager de l'examen de la végétation plusieurs associations. Nous examinerons successivement: le Querceto-Carpinetum montanum et ses facies, le Fagetum tilietostm, un QuercetoFagetum de montagne, des bois proches des Querceto-Lithospermetum et Quercetum pubescentis, le Quercetum sessiliflorae et le Quercetum roboris appauvris.

\section{1 - Le Querceto-Carpinetum montanum Lemée.}

Cette sous-association du Querceto-Carpinetum est une projection montagnarde décrite d'ahord dans la Comté d'Auvergne par I.F.MÉ.F, puis par le méme auteur dans les Monts Dómes. Il s'agit d'un taillis sous futaie riche en espèces. Nous avons noté:

\section{Caractéristiques d'association et Différenticlles} de sous-association.

Carpinus betulus I., 2;2 - Geranium nodosum I. 2:2 - Potentilla fragariastrum Ehrh. 1:2 - Rauunculus auricomus I. + - Vinca minor + - Lilium martagon L. +.

\section{Frarino-Carpinion.}

Fraximus cxcelsior L. 1:2 - Evonymus curopaess 1.. ssp. sulgaris Mill. + - Geum urbanum L. +.

\section{Fagion et Fagetalia.}

Euphorbia dulcis I., 2-1; Miliun cffusum 1. 1-2; Asperula odorata L. 1-3; Ancmone nemorosa L. 1-1; Stellaria holostea 1. 1-1; Pulmonaria affinis Jord. ssp. eu-affinis P.F. + ; Phyteuma spicatum 
L. ssp. ochroleucum Schulze + : Epilobium montanum L. + : Vicia sepium L. + : Viola silvatica Koch +; Galcobdolon luteum Huds. + ; Neottia nidus-avis Rich. + .

\section{Espèces compagnes.}

Quercus pedunculata Ehrh. +; Acer campestre 1. + : Acer platanoides L. + ; Rubus sp. 1-2; Cornus sanguinea L. 1-1; Viburmum lantana L. 1-1; Cratagews oxyacantha $\mathrm{L}$. et C. monogyna Jacq 1-1: Ribes alpimum L. 1-1; Poa nemoralis L. 2-3; Hieracium gr. murorum L. 1-2; Orobus tuberosus L. (- Lathyrus montanus Bernh.) 1-1; Festuca heterophylla Lmk, +; Lusula pilosa Willd. +; $\times$ Luzula borreri Bromf. + : Ranunculus breyninus Crantz + : Solidago virga-aurea I. var. latifolia Koch +: Melampyrum pratense L. +; Allum ursinum L. X; Conopodium denudatum Koch. $+$

(Nous n’avons pas relevé les muscinées).

Ce relevé se rapproche beaucoup du Querceto-Catpinetum montanum décrit par Lemés dans la Comté d'Auvergne. Remarquons cependant l'absence de Ccrasus avium et d'/lex aquifolium dans cette partic des hois de Courgoul.

Nous avons rencontré sur une pente très humide un peuplement abondant d'Equisetum hyeuale L. que lon peut considérer comme facies de la sous-association. Il existe un facies comparable dans la Comté près de Mercurol.

\section{2 - Le Fageto-Scilletum tilietosum.}

C'est le Fagetum gallicum tilietosum de LemÉE 1946. Quelques ilots forestiers parmi le Querceto-Carpinetum précedent semblent bien appartenir a cette sous-association. Trois individus d'association nous ont fourni (la notation chiffrée représente le degré de présence):

\section{Differentielles de sous-association.}

Tilia platyphyllos Scop. 3 - Alliwm ursinum L. 3 - Geranitm nodosum L. 2- Galium mollugo 1.. ssp, clatum Thuill. 2 - Elymus curopers I. 1.

Caractéristiques de Passociation.

Faqus silvatica I. 3 - Pulmonaria affinis Jord. ssp. cu-affinis P.F. 3 - Polygonatum verticillatum A11. 2 - Epilobium montanum L. 2 Conopodium denudatum Koch 1. 
Fagion et Fagetalia silvaticae.

Asperula odorata I. 3 - Phyteuma spicatum L. ssp. ochroleucum Schulze 3 - Dentaria pinnata Lmk. 3 - Poa nemoralis 2 - Prenanthes purpurea L. 2 - Galeobdolon luteum Huds. 2 - Euphorbia amygdaloides L. 2 - Paris quadrifolia L. 1 - Festuca silvatica Vill. 1 Vicia sepium L. 1 - Myosotis silvatica Hoffm, 1 - Milimm effusum L. 1 - Actaea spicata 1.. 1.

Compaynes.

Quercus pedunculata Ehrh. 1 - Acer platanoides L. 1 - Fiola silvatica Koch 3 - Oxalis acetosella L. 3 - Dryopteris filix-mas Schott. 2 - Hieracium gr. murorum L. 1 - Athyritm filix-foemina Roth. 1 - Poa chaixii Vill. 1 - Senecio cacaliaster L.mk. 1....

Lat proximité du Fageto-Scilletum enrichit légérement nos individus d'association en éléments appartenant à la flore de la hètraie par rapport aux relevés donnés par Lemée de la Comté. I a composition est assez différente de celle du Querceto-Carpinetum montanum.

\section{3 - Le « Querceto-Fagetum de montagne ».}

Nous sommes en possession de deux relevés que nous avons en une certaine difficulté à intégrer dans une association déjà décrite. Après comparaison avec les listes données par divers auteurs, notamment en ce qui concerne les Querceto-Carpinetum et le FagetoScilletum tilietosum, il nous a semblé que l'on devrait les rapprocher du Querceto-Fagetum décrit par Lemée dans le Perche. La présence d'espèces montagnardes permettrait peut-être d'en faire une sous-association " montanum " si un plus grand nombre de relevés vient confirmer ceux que nous donnons aujourd'hui.

Caractéristiques d'association (Notation en degrés de fréquence).

Fayus silvatica L. 2 - Quercus sessiliflora Salibs. 2 - Veronica montana I. 2 - Circaea lutetiana I. 2 - Melica uniflora Retz 1 - Milium effusum L. 1 - Anemone ranunculoides Wid, 1.

Fagion et Fagetalia.

Epilobium montanum L. 2 - Viola silvatica Koch. 2 - Prenanthes purpurea L. 2 - Poa nemoralis L, 2 - Pulmonaria affinis Jord. ssp. 1 - Carex silvatica Huds. 1 - Sanicula europaca 1.. 1.

Quercion et Quercetalia.

Solidago virga-aurea I., 2 - Rubus sp. 2 - Melampyrum pratense L. 1 -Orobus tuberosus L., 1 - Hieracium murorum L. 1 - Deschampsia flexuosa Griseb. 1 - Festuca heterophylla Lmk. 1. 
Espèces compagnes.

Litium martagon L. 2 - Ajuga reptans L. 2 - Campanula persicaefolia L, 1 - Knautia ci. silvatica Duby 1 - Polygonatum multiflorum L. 1.

On note l'absence d'espéces du Fraxino-Carpinion et des caractéristiques du Querceto-Carpinctum.

\section{4 - Le Querceto-Carpinetum holcetosum mollis Leméc.}

Nous plaçons immédiatement à còté du Querceto-Fagetum montanum la sous-association acidiphile du Querceto-Carpinetum. Cela permettra les comparaisons entre les Monts Dômes où le QuercetoCarpinetum holcetosum mollis occupe les terrains acides du rebord du plateau, et les Monts Dores plus riches en associations de transition.

Différenticlles de sous-association.

Veronica officinalis L. V; 0.6 - Campanula rotundifolia L. ssp. III ; 0,4 - Holcus mollis L. III; 0,4 - Ranunculus breyninus Crantz ssp. nemorosus D.C et ssp. polyanthemoides Bor. II; 0.2 - Melitis melissophyllum 1. 11; 0,1 - Melampyrum cristatum 1. I: 0.1 .

Caracteristiques d'association.

Carpinus betulus I. V; 1,6 - Cerasus avium L. I: 0,2 - Potentilla fragariastrum Ehrh. V; 0,4 - Ranunculus auricomus L. IV; 0,3 Vinca minor L. II;0,2 - Adoxa moschatellina L. 11;0,1 - Conopodium denudatum Koch $\mathrm{I} ; 0,1$.

Fraxino-Carpinion.

Carpinus betulus L. V; 1,6 - Cerasus avimu I. I; 0,2 - Potentilla fragariastrum Ehrh. $\mathrm{V} ; 0,4$ - Ranunculus auricomus I. IV: 0,3 Vinca minor L. II; 0,2 - Adoxa moschatellina L. II; 0,1 - Conopodium denudatum Koch $\mathrm{I} ; 0,1$.

\section{Fraxino-Carpinion.}

Evonymus europeus I. ssp, vulgaris Mill, III; 0,4-Ficaria verna Huds. II ; 0,4 - Acer pseudo-platanus L. I; 0.2 - Chrysosplenium alternifolium L. I; 0,1.

Fagion et Fagetalia.

Prenanthes purpurea L. II; 0,3 - Viola silvatica Koch I; 0,2 - Asperula odorata L. I; 0,1 - Phyteuma spicatum L. ssp. ochroleucum Schulze I; 0,1 - Euphorbia dulcis L. I; 0,1. 
Quercion et Quercetalia.

Melampyrum pratense L. V; 0,3 - Teucrim scorodonia L. IV: 0,3 - Hypericum pulchrum I. IV; 0,2 - Orobus tuberosus L. III ; 0,6 - Solidago virga-aurea L. III ; 0,2 - Quercus sessiliflora Salisb. II : 0,2 - Hicracium murorum I. II ; 0,2 - Betonica officinalis Trev. II : 0,1 - Deschampsia flexuosa Gris. I; 0,3 - Hieracium borcale Fries I; 0,1 - Hieracimm nemorense Zahn. I: 0,1 - Convallaria maialis $\mathrm{L} .1$ I; 0,1 .

Espèces compagnes.

Acer campestre I., III ; 0,3 - Sorbus aria Crtz. II ; 0,4 - Cratacgus sp. I; 0,1 - Sorbus torminalis Crtz. I; 0,1 - Poa nemoralis L. III : 0,2 - Luzula pilosa Willd. III ; 0,2 - Luzula forsterii D.C. II ; 0,2 Veronica montana L. I; 0,1 - Campanula persicaefolia I. I; 0,1 Ajuga reptan,s L. 1: 0,1 - Hedera helix L. I; 0,1 - Veronica chamaedrys L. I; 0,1.

(12 relevés).

\section{5 - Le Querceto-Lithospermetum Br. B1.}

Les expositions sud portent assez fréquemment de La Tourette à Saurier des individus d'association du Querceto-Lithospermetum. Nous avons choisi ceux qui sont le plus près des éclaircies et qui nous ont paru les plus caractéristiques pour relever:

\section{Caractóristiques d'association* .}

Quercus lanuginosa Lmk. 8 - Pirus commmis L. ssp, achras Gaertn, 6 - Lathyrus niger Bernh, 8 - Mellittis melissophyllum L. 7 - Lithospermum purpureo-caeruleum L. 7 - Campanula persicaefolia L. 5 - Vincetoxicum officinale Moench ssp. laxum G. et G. 3-Helleborus foetidus L. 3-Digitalis lutea L. 2 - Hypericum montamum L. 1 .

Quercion pubescenti-sessiliflorae.

Ligustrum vulgare L. 2 - Sorbus aria Crantz 1 - Anthericum liliago L. 6 - Trifolium medium L. 4-Polygonatum officinale All. 3 Peucedanum cervaria Lapeyr. 1 - Laserpitium latifolium I. 1.

Fagion et Fagetalia.

Carpinus betulus L. 4-Cerasus avium L. 1 - Fraxinus excelsior L. 1 - Euonymus vulgaris Mill, 3 - Rosa arvensis L. ssp. 2 - Rosa cf. jundsillii 1 - Hedera helix I. 7 - Pulmonaria affinis Jord. ssp ; eu-

* Notation en degré de fréquence. 
affinis P.F. 6 - Anemone nemorosa I. 7 - Poa nemoralis L. 6 Melica uniflora Retz 6 - Conopodium denudatum Koch 6 - Asperula odorata L. 5 - Vicia sepium L. 3 - Euphorbia dulcis L. 2 - Geranitu nodosum L. 2 - Carex silvatica Huds. 1.

Espèces compagnes.

Quercus sessiliflora Ehrh. 5 - Acer campestre L. 1 - Crataegus monogyna Jacq. 2 - Lonicera xylosteum L. 1 - Orobus tuberosu,s L., 5 Brachypodium pinnatum I. 4 - Geranium hidum I. 2 - Cephalanthera xiphophyllum Rchb, 2 - Trifolium rubens L. 2 - Trifolium agrarium Sreb. 1 - Trifolium glomeratum L. 1 - Hypericum hirsutum L. I - Galium parisiense L. ssp. 1 - Teucrium scorodonia L. - Sedum maximum Hoffm. I - Veronica teucrium L. 1 - Tamus communis L I - Campanula trachelium L. 1.

La composition de cette association est voisine de celle des individus d'association décrits par LEMĖE dans la Comté d'Auvergne. 11 faut cependant noter l'existence d'une sorte de vicariance, certaines espéces préférant le sol volcanique de la Conté et d'autres du même genre s'accommodant mieux du sol granitique de notre région. C'est le cas, semble-t-il, pour Cephalantera xiphophyllum et $C$. rubra, Geranium lucidum et $G$. sanguineum; il y a là un intéressant sujet de recherches.

\section{6 - Le Quercetum pubescentis fragmentaire.}

Au-dessus des vignes abandonnées des environs de Saurier, on observe des taillis d'arbres tortueux et mal venus. II semble s'agir de lambeaux de Quercetum puhescentis.

Caractéristiques d'association.

Quercus lanuginosa L.mk. 3 - Lathyrus niger Bernh. 3 - Pirus communis L. ssp, achras Gaertn, 2 - Trifolitum rubens 1., 2 - Melampyrum cristatum I., 1 - Inula salicina I. 1 - Pencedanum cervaria Lapeyr., 1

Quercion pubescentis-sessiliflorae,

Sorbus torminalis Crantz 2 - Melittis melissophyllum L. 2 - Bupleurum falcatum L. 1 - Vincetoxicum officinale Moench 1 - Viburnum lantana L. 1 - Tamus communis L. 1 - Cornus sanguinea I. (jeune pousse) 1 - Inula conyza D.C. 1.

\section{Fagion et Fagetalia.}

Fraxinus excelsior L. 1 - Carpinus betuluș L. 1 - Fuonymus vulgaris Mill. 1 - Asperula odorata L. 2 - Vicia sepium L. 2 - Poa nemoralis L. 1 - Melica uniflora Retz 1 - Stellaria holostea L. 1 - Euphorbia anygdaloides L, 1 - Mercurialis perennis L, 1 - Pulmonaria affinis L. ssp, eu-affinis P.F, 1. 
Espèces compagnes.

Robinia pseudacacia L, 2 - Salix of. purpurea L. (S. helix L.) 2 Rubus sp. 2 - Vitis sativa D,C. 1 - Brachypodiun pimatum Pal. 3 Geranium robertianum L. 2 - Poa nemoralis L. 2 - Trifolium rubens L. 2 - Galium mollugo L. ssp. 2 - Valerianella olitoria Poll. 1 Teucrium scorodonia L. 1 - Hedera helix L. 1 - Fragaria vesca L. 1 - Ticia hirsuta Gray 1 - Hypericum hirsutum L. 1 - Trifolium gloneratum L., 1.

\section{7 - Le Quercetum roboris appauvri.}

Nous n'avons pas accompli de relevés dans quelques maigres lambeaux de Quercetum roboris. La flore du sous-bois présente un mélange d'espéces des associations voisines, notamment du Quercetum sessiliflorae et du Quercetum pubescentis (plus rare). Quelques différentielles petuvent cependant être reconnues: Quercus pedunculata (par son abondance), Pcucedanum cervaria et $P$. gallicum, Epipactis latifolia (rare). II ne parait pas y avoir une grande différence avec le Quercetum rohoris classique du Bassin Parisien.

\section{8 - Le Quercetum sessiliflorae Allorge 1924.}

Il s'agit là d’une association bien mieux représentée à la base du versant est des Dores. Les chênes sessiles sont généralement mal venus et de faible taille. Nous avons noté:

\section{Caractéristiques d'association*}

Quercus sessiliflora Salisb. 11 - Deschampsia flerwosa Griseb. 8 Veronica officinalis L. 6 -Vaccinium myrtillus L. 6-Hicracium gr. murormm L. 5 - Hienacium praecox Sch. Bip. ssp. conjugatum A.T. et ssp. pallidulum Zathn 4 - Festuca heterophvlla L. 3-Campanula rotundifolia L. 3 - Pteridium aquilinum I. 2 -Leucolryum glascums Hamp, 1.

Quercion et Quercetalia sessiliflorae.

Melampyrum pratense L. 10 - Teucrium scorodonia L. 9 - Solidago virga-aurea L., 9 - Sorbus aria Crtz. 7 - Orobus tuberosus I., 7 Convallaria maialis L. 6 - Hieracium umbellatum L. et H. sabaudum Zahn 6- Melampyrum sileaticum L. 2- Hypericum pulchrus L. 2 .

Fagion et Fagetalia silvaticae.

Prenanthes purpurea L. 3 - Poa nemoralis L. 1.

* Notation en degré de présence. 


\section{Espèces des Querceto-Fagetea.}

Anemone nemorosa L, 2 Viola silvatica Koch 2 - Bromus asper L. 1.

\section{Espèces compagnes.}

Rubus sp. 6. - Lusula maxima D.C. 6-Holcus mollis L. 5 - Ranunculus breyninus Crtz. 4 - Orobus tuberosus L. 4. - Phyteuna spicatum ssp. 2 - Betonica officinalis Trev. 2 - Ramunculus auricomus L. 2 - Luzula forsterit D.C. 2 - Luzula pilosa Willd, 2 - Juniperus comunnis I. I - Melittis melissophyllum L. 1 - Euphorbia anygdaloides L. 1 - Betonica officinalis Trev. ssp, serotinus Host. 1 Hedera helix L. 1-Galium satatile G. et G. 1 - Potentilla tormentilla L. 1 - Calamagrostis epigeios Roth 1 - Poa chaixii Vill, 1 Agropyrum repens P.B. 1 - Alliaria officinalis Andrz. 1 - Lunaria rediza 1. 1 - Agrostis nulgaris L. I - Ribes alpinum L. 1. (Hypnum cupressiforme - Scleropodium purum - Hypum splendens - Hyp num triquetrum - Dicranum scoparium - ...)

(11 relevés - les mousses nont été nuties que pattiellement).

\section{9 - Les rapports de ces forêts entre elles et avec les hêtraies.}

Quels sont done les rapports des divers types de hetraies tels que nous les avons définis plus haut avec ces forèts de transition on de l'étage inférieur? Il semble que nous devions dégager deux grands groupes de forèts, liés aux conditions du milieu, surtout édaphiques, bien que ces groupes ne correspondent exactenent à aucune subdivision phytosociologique.

Les forêts sur sol brun ou sur sol peu lessivé (au-densus de $\mathrm{pH}^{5}$ ) renferment à l'étage du hêtre le Fageto-Scilletum et le Fagetol.uzuletum. A l'étage "du chene " on y rencontre, i mesure que l'altitude et l'humidité augmentent, le Querceto-Buxetum (présent dans la plaine de Montbrison par exemple) le Querceto-I.ithospermetum, le Querceto-Carpinetum, le Querceto-Carpinetum montanum et le Querceto-Fagetum montanum, le Fageto-Silletum tilietosum enfin. Les forêts sur sol nettement lessivé ou sur sol podzolique montrent le Fageto-Deschampsietum et ses sous-associations et plus bas le Quercetum roboris, le Quercetum sessiliflorae, le Querceto-Carpinetum holcetosum mollis, LEMÉ dans son étude sur la Comté d'Auvergne avait mis en évidence une partie de cette succession altitudinale. Les conditions écologiques plus favorables de notre région nous ont permis de confirmer les résultats de cet auteur et de les étendre légèrement. Nous avons donc à examiner deux problèmes, comment les associations se répartissent-elles à l'intérieur de chaque groupe écologique et comment les deux groupes définis se succèdent-ils? 
Le Querceto-Buxetum doit son existence dans le Massif Central à des conditions de température particulièrement élevées. SALANon qui l'a étudié aux environs de Montbrison insiste sur ces conditions: " le buis marque une préférence pour les lieux très chauds et très secs $n$. Il semble que les stations de la Conté où l'on rencontre le Querceto-Buxetum aient les mèmes caractères. Le Querceto-Lithospermetum nous parait avoir une origine plus complexe, et nous retrouvons sensiblement les conclusions de LesḱE. Dans la vallée de Courgoul, le Querceto-Lithospermetum se rencontre dans des lieux secs à exposition sud, donc ensoleillés, où l'action présente de l'homme semble tout à fait négligeable si mème elle existe (difficulté d'accès) et en des lieux plus proches des routes et des bâtiments d'exploitation où l'action de l'homme est très forte. Dans ce cas, le Querceto-Lithospermetum correspond à une association succédant assez rapidement à des coupes ou à une exploitation abusive de la forêt. A lopposé, les lieux les plus humides tels que les fonds de vallées ou les ruisseaux sont abondants portent une aulnaie plus ou moins rudéralisée par les touristes et les pêcheurs nombreux. Le Quercetum pubescentis fragmentaire est une association xérophile qui colonise les pentes chaudes et notamment les vignes abandonnées fréquentes dans la région. Cet ahandon peut ètre daté de l'apparition du phylloxera, c'est-à-dire de la fin du XIxe siècle. Notons que ces vignes dépassaient 800 mètres dans la vallée de Courgoul. Il importe enfin de ne pas négliger le rôle des éboulis qui détruisent la végétation. Ces éboulis sont recouverts par une végétation xérothẻrmique relayée après une dizaine d'années par des Querceto-Lithospermetum et des Quercetum pubescentis fragmentaires. L'évolution ultérieure est variable. Le Querceto-Carpinetum de plaine n'existe pas dans notre dition où il est remplacé par sa variante montagnarde, le Querceto-Carpinetum montanum. Cette sous-association recouvre la plus grande partie des pentes entre 600 et 900 metres. Sil composition se rapproche beaucoup de celle des forêts du rebord est des Dômes. Elle est relayée en altitude par une sous-association du Fageto-Scilletum lilio-hyacinthii dont nous avons reconnu des individus entre 700 et 1000 metres, Au-dessus, le Fageto-Scilletum et ses sous-associations d'altitude. Nous considérons le Fageto-Scilletum tilietosum comme le pendant du Fageto-Scilletum allietosum de la limite supérieure de la forèt. Il s'agit de "groupements permanents " au sens de Guinocher, c'est-àdire de formations à composition déterminée par les conditions de milieu - ici vraisemblablement la température - et restant stables, sans que l'on puisse les qualifier de climax. Le climax nous parait en effet être entre 600 et 1000 mètres, le Querceto-Carpinetum.

Les associations du deuxième groupe de forêts sont toutes acidiphiles et elles ont pour caractère commun de dériver de celles du premier groupe. Le seuil biologique de $\mathrm{pH} 5$ dont nous avons parlé à propos des hêtraies se retrouve ici à une altitude plus basse et 
joue le même rỏle important dans l'évolution de la végétation. Le Quercetum roboris - bien fragmentaire daus notre dition il est vrai - se montre moins acidiphile et plus umbrophile que le Quercetum sessiliflorae. II $\mathrm{y}$ jouerait un róle analogue au Fageto-Luzuletum de l'étage supérieur tout en étant un peu plus apte à coloniser les régions humides. Le Quercetum sessiliflorae, lui, peut être considéré comme l'homologue du Fageto-Deschampsietum. C'est la forêt qui occupe les régions les plus pauvres de l'étage du chène. $\mathrm{C}_{e}$ pendant, nous ne retrouvons pas une $"$ autodestruction $x$ de la forêt par envahissement d'espèces du Calluno-Genistetion. En effet, l'altitude est nettement moindre et les pluies sont moins abondantes. n'entrainant pas un lessivage aussi poussé du sol. Remarquons que dans la région de Lezoux à climat plus atlantique et à pluties plus fréquentes, Cilassagne (1936) a décrit sur des sols très podzolisés et à alios épais une "lande à Calluna n qui tend a détruire la forèt et cet auteur parle même d'une "évolution naturelle de la forèt qui forme un sol qui lui est nuicible ".. Au-dessus de l'altitude optimum de la chênaie de chẻnes sessiles, nous rencontrons deux groupements. Le Querceto-Fagetum montanum occupe les lieux plus humides que le Querceto-Carpinetum montanum. Nos ohservations sont trop restreintes pour nous permettre de retracer leurs relations dynamiques. Nous ne pensons cependant pas que laction de l'homme ait une grande importance. Le Querceto-Carpinetum holcetosum mollis est la sous-association acidiphile du Querceto-Carpinetum. Fréquent dans les Domes, ce groupement se retrouve à la base des Dores, I.à on voit nettement qu'il s'intercale entre le Quercetum sessiliflorae et le Fageto-Deschampsietum.

Nous avons classé sur un schéma (fig. 6 et 6 bis) les positions de relation possibles de ces types de foréts. Les abréviations représentent: $\mathrm{S}=$ lisière à Sorbiers - F.S. = Fageto-Scilletum - F.S. Ath = hêtraie à fougères - F.S.All. = hêtraie à Allium - F.S.T. = hêtraie à tilleul - F.S.E = hétraie à prêles- Q.C.M. = Querceto-Carpinetum montanum - Q.C. $=$ Querceto-Carpinetum facies ì préles - A1. - Aulnaie - Q.L. = Querceto Lithospermetum Q.B. = Querceto-Buxetum - et dans l'autre série, J. = Zone it Juniperus - A. - Sapinières - F.D. = Fageto-Deschampsietum F.D.V. = Fageto-Deschampsietum Vaccinietosum - F.D.H. = Fageto-Deschampsietum hylocomietosum - Q.F.M. - Querceto-Fagetum montanum - Q.C.H. - Querceto-Carpitenum holcetosum mollis - Q.S. - Quercetum sessiliflorae - Q.R. = Quercetum roboris.

Comment répartir dans un cadre phytosociologique toutes ces forêts? Nous avons pour cela employé la représentation tétraédrique proposée dans la première partie (fig. 7). La projection des points représentatifs sur le plan ABC (Fagion, Quercion, Querceto-Fagetea) nous permet de reconnaitre immédiatement les deux grands groupes, celui des forèts acidiphiles et celui des forêts qui ne le 


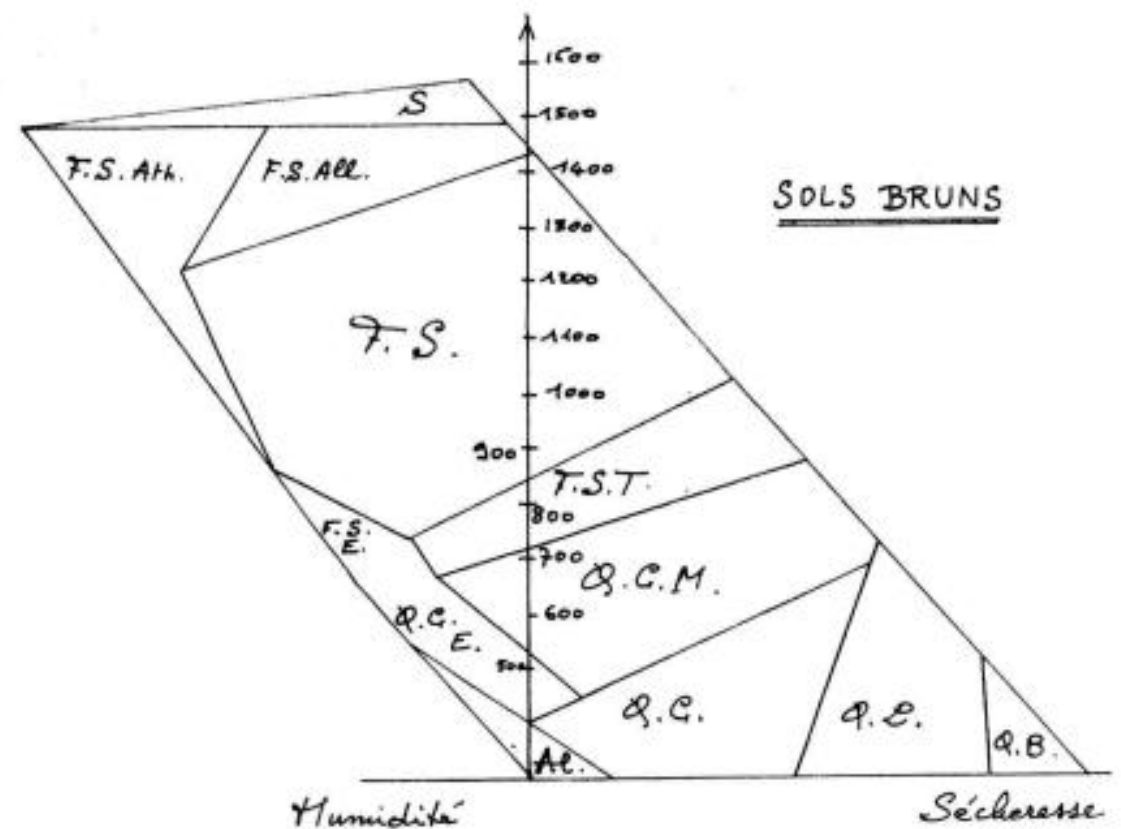

FIG. 6.

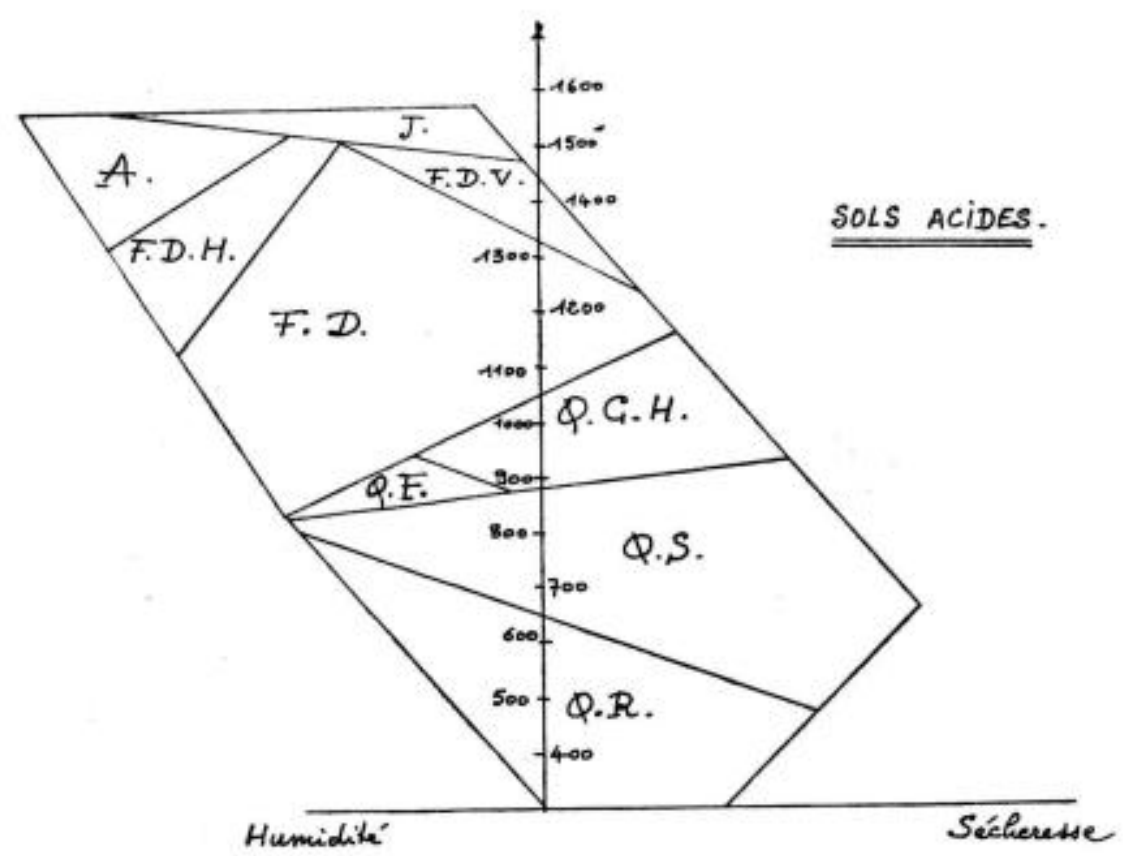

FIG. 6 bis. 

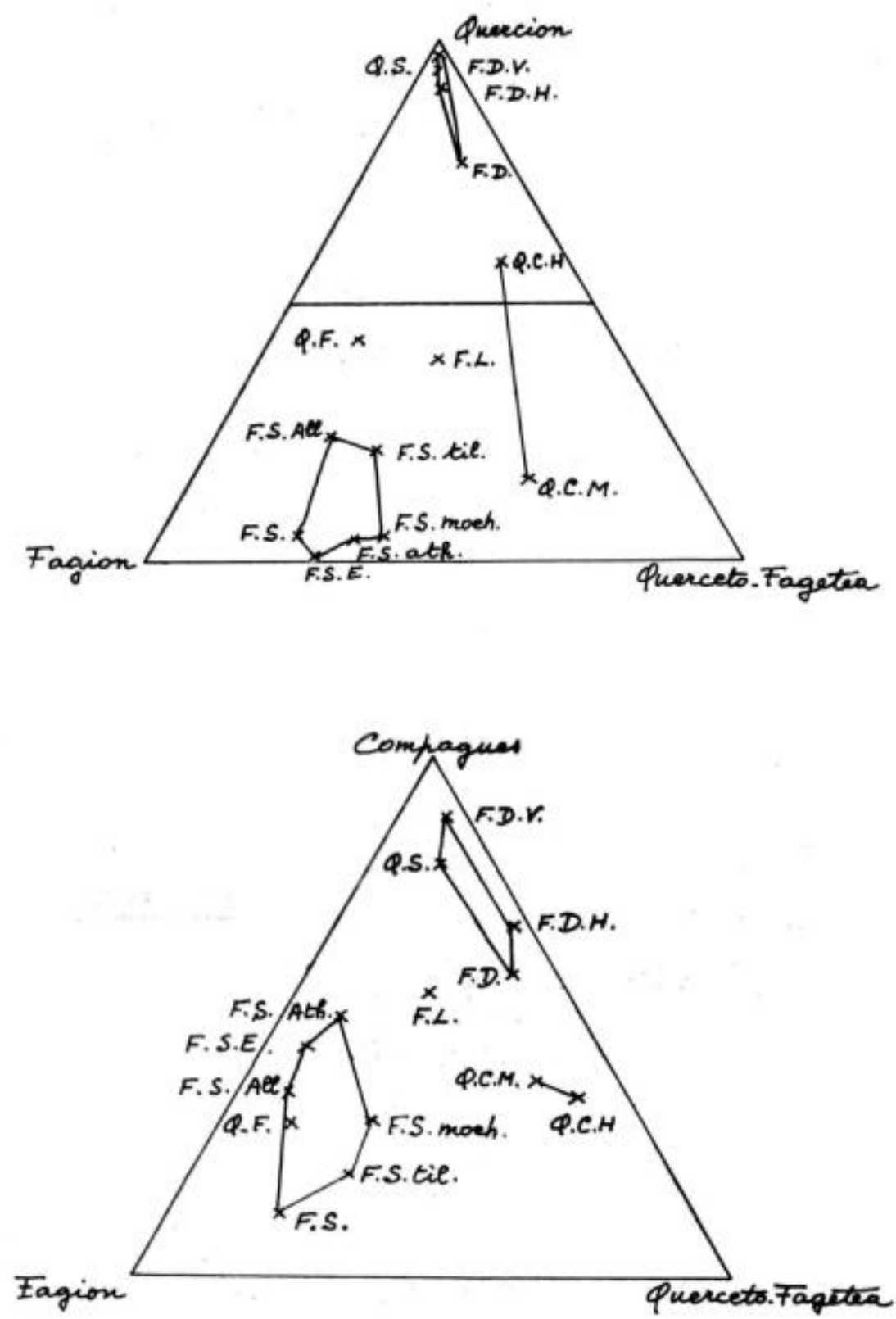

Fig. 7. 
sont pas, La proximité du Quercetum sessiliflorae et du FagetoDeschampsietum et de ses sous-associations n'est pas le fait du hasard mais traduit les similitudes étroites qui les unissent, autrement dit, le Fageto-Deschampsietum est le vicariant d'altitude du Quercetum sessiliflorae. La projection sur le plan ADC nous le confirme pleinement. D'un autre cóté, la projection $\mathrm{ABC}$ nous montre que le Fageto-Scilletum tilietosun est bien étroitement lié aux autres groupements de cette association. ("est la forme du climax qui est lice à une altitude inférieure à celle du climax Fageto-Scilletum de " l'étage du hètre n et qui est à une altitude supérieure à celle du climax Querceto-Carpinetum. Ce n'est pas un groupement de transition mais la "variété " du climax imposée par le milieu. Nous avions noté la difficulté de classer le Querceto-Fagetum montanum il apparait sur les plans de projection comme appartenant à l'ordre des Fagetalia mais avec une " tendance "Quercetalia, il nous parait qu'il n'est qu'un groupement de transition entre les chẻnaies et les hetraies du type Fageto-Scilletum. I.es Querceto-Carpinetum qui se regroupent bien sur le plan $\mathrm{ADC}$ sont plus lointains sur le plan $\triangle B C$ au point de montrer une appartenance aux Fagetalia pour le Querceto-Carpinetum montanum et aux Quercetalia pour le Querceto-Carpinetum holcetosum mollis - c'est mettre en évidence toute la difficulté de classer certains groupements.

Dans nos conclusions, nous envisagerons quelles peuvent itre les conséquences de lexploitation sur ces grompements.

\section{F - Plantations et bois de coniferes}

Nous n'avens pas encore parlé des foréts de sapin. Si elles sont nombreuses et belles sur le versant ouest des Dores, il n'existe atucune foret naturelle de sapin sur le versant est que nous avons examiné, Une telle anomalie peut surprendre, surtout après les résultats de l'analyse pollinique. En effet, il semble bien établi que le sapin est arrivé avant le hètre dans la région des Dores et qu'il a d'abord pris tune nette prépondérance. I.humidité un peu plus forte sur le versant occidental l'a peut-être favorisé, mais les conditions climatiques du versint oriental sont bien suffisantes pour lui permettre de prospérer: aussi pensons-nous qu'il convient de rechercher ailleurs les causes de sa disparition. Nous avons signalé qu'à la fin du $\mathrm{xv}^{\mathrm{e}}$ siècle les hêtraies étaient à pet près détruites par le pacage et une exploitation intensive et désordonnée. II semble que les forêts de sapin du versant ouest aient été respectées, car il s'agissait de propriétés seigneuriales ou ecclésiastiques et elles ont échappé aux coupes à blanc. En 1726, on tenta d'exploiter la futaie pout en tirer des mâts de vaisseaux, ce qui indique la présence de beaux arbres. Ce serait donc l'action de l'homme favorisant le sapin sur le versant ouest et détruisant la forêt sur l'autre versant, qui pourrait expliquer cette répartition différente des deux essences. II 
est d'ailleurs remarquable de constater la ressemblance entre les strates arbustives et herbacées des sapinières et des hètraies. I.a setule différence entre I" "Abietetum " et le Fageto-Deschampsietum consiste en la présence de Lusula flavescons Gaud. dans la forèt de sapin du Lioran. Si l'on ajoute que Listera cordata $\mathrm{R}$. Br. exclusive de la sapinière pour I.vQUET, se rencontre dans les bois d'épicea des Tatra en Pologne (SzAFER - 1923), on conviendra que la différence pratiquement unique réside en l'exclusivité dans la strate arborescente de Fayus silvatica ou d'Abies alha. Gunviek (1956) parlant des forêts d'Auvergne écrit d'ailleurs: "il en est tout autrenent du sapin que souvent on a planté... dans ces conditions, le sapin est définitivement installé en bien des cas et se propage n. Nous estimons que le problème parfois posé du climax, hètraie out sapinière, est un faux problème dans les Dores. Il ne s'agit que des différents traitements subis par les forêts primitives, mélanges de hêtre et de sapin. C'est une indication utile pour les reboisements. La forèt mélangée - hètre + sapin - parait en effet la forêt naturelle clans les Dores au-dessus de 900 mètres.

I.es forestiers ont sonvent utilisé l'épicéa (Picéa excelsa I.ink.) dans leurs reboisements. Cet arbre n'est pas spontané dans le Massif central et il ne semble pas que la découverte d'une empreinte de graine ressemblant à celle de Picea puisse remettre en cause cette conclusion. Cependant cet arbre s'accommode rennarquablement bien des conditions climatiques et peu à peu constitue un ensemble proche du. Piccetum montanum galietosum rotundifolii des Alpes, Les exclusives de ce groupement sont encore rares en Auvergne il est vrai, et l'on ne peut guère considérer comme telles que Galium rotundifolium et Hypnum crista-castrensis. T.e hêtre parait devoir, en l'ahsence d'entretien, éliminer l'épicéa car il repousse vigoureusement en sous-bnis. I.FMÉE a ohservé des phénomènes comparables dans la chaine des Dômes.

Le pin sylvestre d'Auvergne se présente de deux manières a priori differentes: la race d'Auvergne est en place et de nombreuses races (Riga, des Vosges, Haguenau...) employées en rebrisements depuis le milien du $\mathrm{x}^{*}$ siècle. I, fcon fait cependant remarquer en ce qui concerne la race d'Auvergne (ses observations concernent les stations actuelles de cette race qui était pratiquement la seule à l'époque où il écrivait) qu'il n'y a pas de végétation spéciale sous un peuplement de pins et que les plantes les plus disparates s'y rencontrent. Il en est de même pour les peuplements récents effectués avec d'autres races. II n'existe donc nas d'association du Pin. Cependant, la rusticité de cet arbre et son amour des stations bien éclairées et chaudes lui permettent de jouer un rỏle important de pionnier dans le dynamisme de la végétation: il crée un sol où peuvent s'installer les hêtres qui poussent à son ombre. Aussi reste-t-il fréquemment dans les plantations de pins des espèces des groupements précédents et la grande luminosité qui règne 
sous ces peuplements permet longtemps la survie de plantes des pelouses sèches que le pin remplace lorsutuil est spontané. C"est ainsi que l'on reconnait inmédiatement un sous-bois de pins à la présence de Brachypodium pinnatum, de Pilosella sp., de Genista pilosa, d'Euphorbia cyparissias, de Scabiosa columbaria... Mais il n'est pas question d'en faire un ensemble spécifique.

\section{CONCLUSIONS GENERALES}

Permettons-nous tout d'abord une conclusion f'ordre méthodologique. Nous avons employé dans l'étude des forcits d'Auvergne des méthodes de l'école de \%urich-Montpellier auxquelles nous avons ajouté des procédés très mathématiques. On aurait pu craindre quainsi nots nous fussions écarté par trop de la réalité des choses. L'étude écologique - microclimatique et pédologique - a pleinement confirmé les résultats obtenus et le découpage floristique. Il est done remarquable de constater que l'emploi de méthodes zuricho-montpelliéraines conduit à la notion de groupes écologiques proches de ceux définis par Duchaufour clans les Vosges par exemple et à la notion de relais altitudinal, c'est-à-dire de "catena n. au sens de Duvignesub. Il ne parait pas interdit d'espérer qu'une précision accrue, par l'emploi de tests statistiques de signification entre autres, permettrait de concilier les points de vue actuellement divers des phytosociologues.

Un second point sur lequel nous ne saurions trop insister est la concordance, le parallélisme parfait que notus avons observé dans l'évolution de la végétation et du sol. Il nous semble qu'il y a là une confirmation des remarquables travaux poursuivis dans ce sens deptis la thèse de Duchnufour. II apparait donc deux grands groupes d'associations dont le déterminisme est d'ordre édaphique. Quelles conclusions pratiques peut-on en déduire pour l'exploitation des forêts?

Nous avons montré que le seuil biologique de $\mathrm{pH} 5$ marquait la limite au delà de laquelle l'évolution du sol dans le sens de la podzolisation, et de la végétation naturelle dans le sens de la dégradation. était irréversible. Il faut donc que l'action des forestiers. sous peine de voir la dégradation à assez brève échéance du sol et de la forêt. s'exerce de manière à empêcher le seuil critique de $\mathrm{pH} 5$ d'ètre atteint. Pour cela, à l'étage du hêtre le Fageto-Tuzuletum marque la limite de reconstitution naturelle du climax FagetoScilletum. La destruction des myrtilles et surtout des callunes s'imposerait en sous-bois at cas où ce setuil serait dépassé. Ia plantation de résineux semble également assez délicate dans le FagetoDeschampsietum. Au cas où la croissance des arbres serait normale en première génération, leur litière ne tarderait pas à acidifier de façon irréparable le sol et la forêt se détrtuirait d'elle-même. 
II ne resterait qu'un Calluno-Gienistetum qui serait pratiquement impossible à reboiser. Un certain équilibre hêtre-sapin et surtout hétre-épicéa semble devoir être entretenu pour conserver un sol de bonne valeur. Une exploitation qui viserait des résultats à trop brève échéance, production intensive de résineux et surtout d'épicéas, se révélerait très préjudiciable, Rappelons qu’il semble bien que la seule action de l'homme a délimité les donaines actuels dut hetre et du sapin dans les Monts Dores.

L'action du forestier parait actuellement assez réduite dans les maigres forèts peu exploitables de la base des Dores et du Cézallier. a l'exception de quelques plantations de pins. I.'homologie avec l'étage supérieur est évidente: il convient de favoriser le FagetoScilletum tilietosum et le Querceto-Carpinetum montanum anx dépens de sa variante acidiphile. Les plantations de pins et plus spécialement de pins d'Auvergne qui résistent bien aux écarts du climat permettent effectivement le meilleur emploi des sols. I at végétation naturelle semble cependant devoir s'emparer assez rapidement de ces peuplements pour y reconstituer des taillis sils ne sont souvent éclaircis en sous-hois. Il est douteux que leur rapport le nermette. Dans un premier stade. lavantage de cette installation en sous-bois est évident: amélioration de l'humus et élagage naturel de la base des résineux. Nous avons cependant observé de nomhreux cas où les hètres avaient totalement étouffé les jeunes pins quatre ou cinq ans après leur plantation.

\section{Note ajoutee al cours d'impression.}

Nous venons de prendre emmaissance de Particle de CARBIFNEk dans a te Hohneck >. Si lon considère que les associations qu'il nomme Luzulo-Fagetum. Hylocomieto-Abietetum et Festuceto-sylvaticae-Fagetum correspondent respectivement à nos Fageto-Luzulefum et Fageto-Deschampsietum à nos FagetoDeschampsictum hylocomietosum et Fageto-Deschampsietum vaccinietosum. et is notre Fageto-Scilletum avec ses sotss-associations, on voit que nous avonexactement le même parallélisme que cet auteur en ce qui concerne les rapports de la végétation et des sols.

\section{BIBLIOGRAPHIE SOMMAIRE}

Agsfonorf, - 1935. Sols de France. Ann. Agr., 5, 3, 333.

Aichisger. - 1954. Static and dynamic consideration in phytosociological research work. $\mathrm{PIII}^{\circ}$ Cong. Int. Bot. (7-8, 87.

n'AlLetzetTe. - 1956. La session extraordinaire de la Société botanique de France en Auvergne. Bull. Soc. Bot. Fr, 103, 36.

- 1962, Clef analytique de la Flore d'Auvergne.

Amosce et Gaussen, - 1934. La hétraie d'Urbasa. Bull. Soc. Bot. Fr. Session extr. Pays Basque, 81.

D'Alverny. - 1907. - I Les hautes-chaumes du Forez. Rezue des Eaur ef Forêts.

- 1910. Géographie botanique des Monts du Forez. Ann. Soc. Bot. Lyon, 35, $153-178$. 
Atgarde. - 1957. Contribution à l'étude des problèmes de l'homogénéité en phytosociologie. Rull, Serz. Carfe Phytos., 11, (I), (II).

Bertraxn, - 1886. Les reboisements dans le Puy de Dóme. Reare des Faw et Forîts.

Botsse de Brack ou CHotichrt. - 1951. Les glaciations de l'Auvergne.

Braun J. - 1915. Etudes sur la végétation méridionale. I. Les Cévennes méridionales: Massif de 1'Aigoual. Arch. Sc. Phys. Nat. Genèce, $4^{*}$ Sér., 30.

Braun-Blanguet J. - 1921. L'origine et le développement des flores dans le Massif Central de France. Bull. Soc. Linn. Lyon, 68 à 76.

- 1926. Etudes phytosociologiques en Auvergne. Le climax complexe des landes alpines. Arvernia, fasc 2.

- 1928. Pflanzensociologie. $(2$ exl., 1951$)$.

Braun-Blanguet et Coll. - 1951. I.es groupements végétaux de la France méditerranéenne.

Bufpault, - 1902. Les foréts de l'Aubrac. Reque des Faux el fiorts.

- 1923. Quelques forets anciennes du Cantal. Resue de Haute-Auzverque, 22. 98 à 119 et 210 ì 241.

Carbiener. - 1963. Les sols du Massif du Hohneck. Leurs rapports avec le tapis végétal, in \& Le Hohneck s, Strasbourg. 1 vol,

Cakles. - 1949. L'équilibre hêtre-sapin sur l'arête orientale du Massif Central, Cong. Ass. Fr. Arranc. So, 96.

Chassagne. - 1956. Inventaire analytique de la flore d'Auvergne et contrées limitrophes des départements voisins. Lechevallier. Paris.

Cuovard, - 1955. La dormance chez le hêtre. C. R. Acad. Agr., 10, 454.

Comars. - 1910. Détermination des intensités lumineuses optimit pour les végétaux, Amer. Soc. Bot, 9, 11.

CZFKanowski, - 1913. Zarysmetod statystycznych. Warsawa.

Dмнг et Hans. - 1949. Homogeneity of plant communities. Studia Botanica Cechostovaca, 10, 159

Ducuavpour. - 1949. Les sols des hètraies. C.R. Acad. Ayr., 10, 390.

- 1954. Evolution des sols forestiers en liaison avec la végétation forestière. Revue forcstièrs, 11, 641.

- 1957. Pédologie. Tableatux descriptifs et analytiques des sols. Feole Nationale des Faux et Forèts.

- 1958. Pédologic. Applications forestières et agricoles

- 1960. Précis de nérlologie. Masson.

Fmrenger, - 1942, Un projet de classification des climats au point de ver Phytogéographique, Bull. Soc, Hist, Nat, Toulouse, 77.

FALINSKI. - 1958. Tablice wspolézymikow ptodolienstiva miedzy xdjeciani fitosocjologicznymi wedlug wzorn Jaccarda i Steinhausa. Acta Soc. Bot, Pol., 27 (1).

Fанаult, - 1901. Les limites supérieures de la végétation forestière et les prairies pseudo-alpines en France. Revue des Fan.t et Forits.

Fischer. - 1944. Statistical methods for research workers, 9* edit.

Fliche et Grandeav. - Recherches physiques et chimiques sur la bruyère commune (Calluna vulgaris). Ann. Sc. Agr. Nancy.

Gaussex, - 1954. Théories et classification des climats et des microclimats. VIII" Cong. Int, Bot, 78, 125.

Grangeox, - 1958. Contribution à Pétude de la fore stampienne de I.imagne. Rer. Sc. Nat. Auv., 24, 41 à 104.

Gunniz, - 1956. Arbres et foréts du Massif central. Rull. Soc. Bot. Fr.. Session extr. Auvergne, 95 à 104 .

Gurvochrt. - 1954. Sur les fondements statistigues de la phytosociologie et quelques-unes de leurs conséquences, Verrof. Geobot. Inst. Rübel., 29, 41.

- 1955. Logique et dynamique du peuplement végétal. Masson.

Gutnochet et Casat. - 1957. Sur lanalyse différentielle de Czekanowski et son applieation à la Phytosociologie. Bull. Serv, Carte Phyto, 11, $2^{2}$ sér., 1 . 
Junc. - 1946. Géologie de l'Auvergne, Mém, Serti, Carte Génl.

KocH. - 1944. Ta phytosociologie de la forèt. Jowm. Forest. Suisse, 2 à 12

KracsE. - 1958. Boden und Pflanzengesellchaften. Handbuch of Physiologid, 3, 824 .

DE. I,мснатғы. - 1961. Fsquisse dune monographie phytosociologique de la vallée de Chatudefour. D.E.S. Clermont.

L.Ecog. - 1835. Le Mont Dore et ses environs. Guide du touriste en Auvergne. 2* livraison.

- 1954. Etudes sur la géographie botanique de l'Europe et en particulier sur la végétation du Plateau Central de la France. Baillère. Paris.

I.Fuḱk. - 1934. Sur l'alliance du hètre, ou Fagion, dans le Perche et le nordouest de la France. C.R. Acod. Sc., 199, 1157.

- 1937. Recherches écologiques sur la végétation du Perche. Thèse. Paris.

- 1946. Etude phytosociologique sur la forêt de la Comté d'Auvergne. Rev: Sc, Not. Auvergue, 12, 22.

- 1953. L'évolution forestière du Massif Central depuis la dernière glaciation. Mélanges géographiques offert à Ph. Arhos. Publ. Fac. leftres Clermont, 3, 7, 189

- 1955. L'évoiution de la forèt française au cours du Quaternaire d’après les analyses polliniques, Revuc forestiare, 6, 442.

- 1956. Le peuplement végétal de lAuvergne. Rev. Sc. Nat. Aurergne. 22. 73 .

I.tover. - 1926. Essai sur la géographie botanique de l'Auvergne. SaintDizier.

Mакту, - 1902. I.ettre au sujet du hètre plincène d'Auvergne. Rezué $d$.Auvergue, Congrès d'Aurillac.

Моюя. - 1958. Zur systematik der Fagetalia. Communic. S.I.G.M.A., n* 6.3.

Mozress. - 1953. Aperçu sur le milieu physique et la flore du massif de l'Aubrac. Bull. Soc, Bot. Fr., 100.

Qusкти, - 1935. L'évolution de la végétation à l'étage de la chénaie daus le Jura méridional. Thèse Paris.

Quezal. - 1952. A propos de foréts de hétres. Bull. Sor. Rot. Fr. Mémoíres Qezzel et Rroux. - 1954. L'étage subalpin dans le Cantal. Vegetatio, 4 , 345.

Rovx, - 1905, Le domaine et la vie du sapin. Anu, Soc. Rot. Lyon.

Sat.ANON, - 1960. Recherches phytosociologiques et écologiques sur les buttes basaltiques des environs de Montbrison (I.oire). D.F.S.Clermont-Ferrand.

Wifsner. - 1907. Der lichtgenuss der Pflanzen. 


\section{RÉSUMÉ}

Cet article est une esquisse phytosociologique des forèts du versant sud des Monts Dores en Auvergne. L'analyse des groupements, effectuée selon les méthodes de l'école de Zurich-Montpellier a été complétée par l'emploi de deux procédés inédits que nous proposons d'appeler "méthode de la différentielle statistique " et "figuration tétraédrique des associations ". Cette analyse a fourni huit associations déjà décrites par les auteurs et neuf́ associations ou sous-associations inédites. On peut les ranger dans deux classes, Fagetalia et Quercetalia, bien que ce groupement ne corresponde pas à la notion immédiate de forêts de hêtres et de forêts de chènes.

L'étude du milieu a été poursuivie simultanément sur deux plans: l'étude microclimatique a fourni des résultats très comparables aux données classiques. L'étude des sols a permis de reconnaitre huit typse de sols dont un est d'une origine assez particulière.

Il faut insister sur le parallélisme très étroit manifesté dans l'évolution de la végétation et celle des sols. On peut considérer deux grands groupes associant d'une part les associations des Fagetalia et les sols à $\mathrm{pH}$ supérieur à 5 ; d'autre part celles des Quercetalia et les sols acides, Le passage se fait au niveau de la hêtraie à Luzules.

Au point de vue pratique, l'influence passée et présente de l'homme sur la forêt est envisagée assez sommairement 


\section{SUMMARY}

This paper is a phytosociological sketch of the forests of the southern slope of the Dore Mounts in Auvergne. The analysis of the associations, conducted according to the Zurich-Montpellier scholl's methods, has been completed by the use of two original procedures we propose to call: " the statistic diffential method " and " the tetraltedral representation of associations $"$. This analysis has already supplied eight associations already described by the authors, and nine original associations or under-associations. They can be classified in two categories, Fagetalia and Quercetalia, though this grouping does not correspond directly to the concepts of beech forests and oak forests.

Site study has been simultaneously conducted on two levels. Soil study has allowed to differentiate eight types of soils, one of which is of a rather peculiar origin.

The very close parallelism apparent between plant and soil evolutions must be underlined. Two main groups can be deferentiated. one relating Fagetalia associations with soils the $\mathrm{pH}$ of which is over 5, and the other relating Quercetalia associations with acid soils. The transition is at the level of beech forest with luzules,

Practically, past and present human influence on the forest is considered rather briefly. 


\section{ZUSAMMENFASSUNG}

Dieser Artikel est ein phytosoziologischer Entwurf der Waldungen, die sich auf der Südseite der $\alpha$ Monts Dores $n$ in der Auvergne, hefinden. Die Errgrüdung der Forstgruppen, vollzogen nach den Lehrsätzen der Schule von Zürich-Montpellier, vurde durch zwei noch nicht an die Offentlichkeit gekommenen Verfahren, ergänzt. Wir werden sie vie folgt nennen: a Methode der statisfischen Ausgleichung n und " bildliche tetraedische Darstellung der Vereinigusgen ". Diese Analysis hat acht von den schon beschriebenen Vereinigungen aufgebracht, und neun Gruppen oder Untergruppen die noch nicht ergänzt sinc. Man kann sie in zwei Klassen aufteilen, Fagetalia und Quercetalia, trotzdem diese Wiedergruppierung gar nicht dem Grundbegriff der Buchen und Eichenwälder entspricht.

Dats Studium des lebensratums wurde gleichzeitig auf zwei Bahnen fortgesetzt: dic mikroklimatische Ergründung brachte ähnliche Ergebnisse als die klassische Methode hervor. Die Bodenergriudung hat Anlass gegeben acht Bodenverschiedenheiten zu entdecken deren eine ganz eigentümliche Herkunft aufweist.

Man nuss den höchst engen Parallelismus zwischen dem Vorgang der Vegetation und der Bodenformung unterstreichen. Man kann da zwei Hauptgruppen in Betracht ziehen: einerseits die Fagetalia mit Bodenbestand über $\mathrm{pH} 5$; andererseits die Quercetalia mit sauregehaltigem Boden. Der Ubergang trifft man auf gleicher Höhe des Buchenbestands mit Luzulaarten.

Auf praktischem Standpunkt aus, wird die vergangene und zuständige Einwirkung des Menschen auf den Wald nur flüchtig ins Auge gefasst. 